\title{
EVALUATING METHODS FOR DETERMINING WATER USE IN THE HIGH PLAINS IN PARTS OF COLORADO, KANSAS, NEBRASKA, NEW MEXICO, OKLAHOMA, SOUTH DAKOTA, TEXAS, AND WYOMING; 1979
}

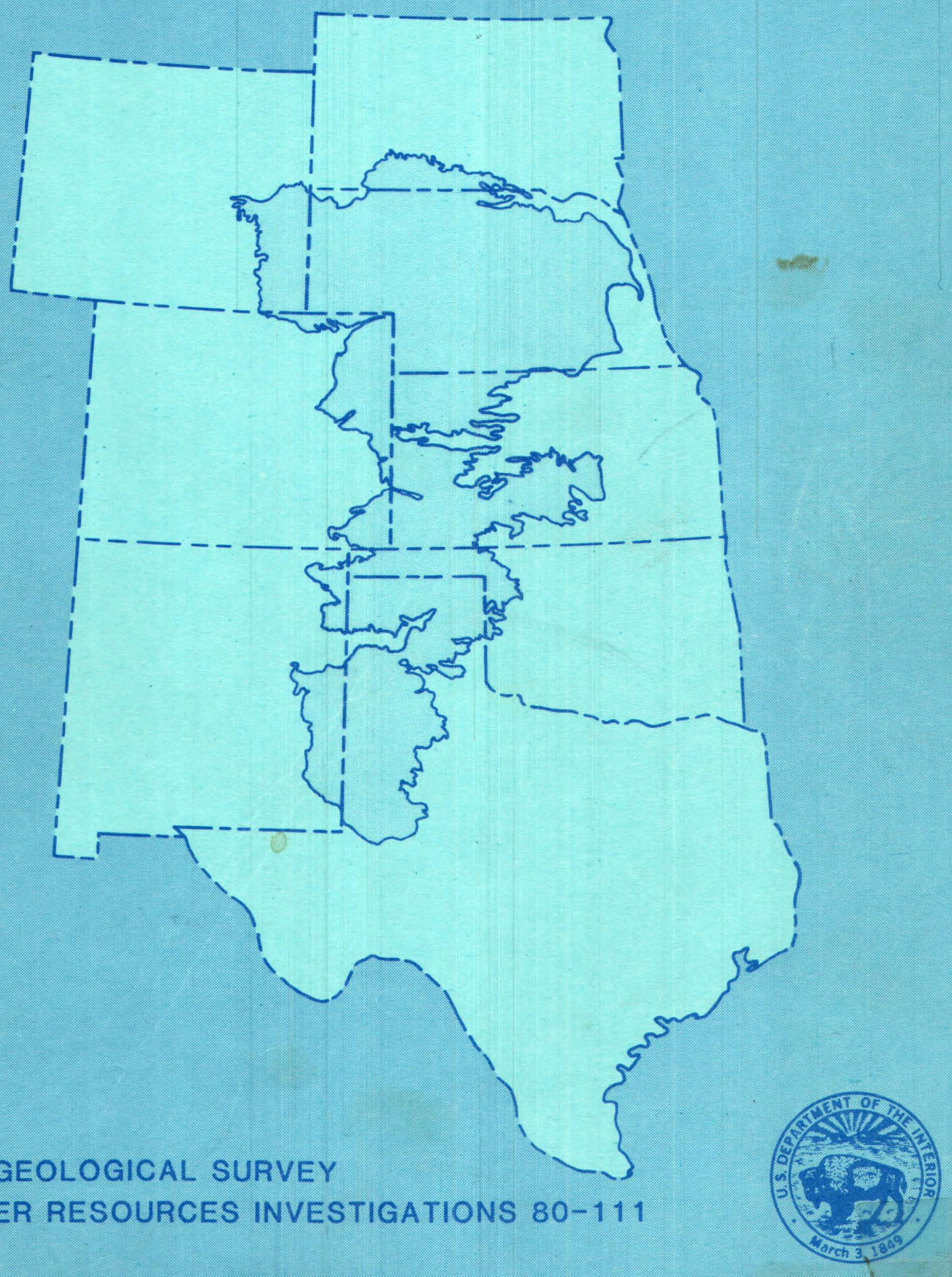

U.S. GEOLOGICAL SURVEY

WATER RESOURCES INVESTIGATIONS 80-111 
UNITED STATES

DEPARTMENT OF THE INTERIOR

GEOLOGICAL SURVEY

EVALUATING METHODS FOR DETERMINING WATER USE IN THE HIGH PLAINS

IN PARTS OF COLORADO, KANSAS, NEBRASKA, NEW MEXICO, OKLAHOMA,

SOUTH DAKOTA, TEXAS, AND WYOMING; 1979

By Frederick J. Heimes and Richard R. Luckey

U.S. GEOLOGICAL SURVEY

Water-Resources Investigations $80-111$

Denver, Colorado 
UNITED STATES DEPARTMENT OF THE INTERIOR

CECIL D. ANDRUS, Secretary

GEOLOGICAL SURVEY

H. William Menard, Director

For additional information write to:

U.S. Geological Survey

Water Resources Division

Box 25046, Mail Stop 412,

Denver Federal Center

Denver, Colorado 80225 
Conversion table-

Abstract-

Introduction-

High Plains description-_- 2

Water use- 2

Approach- 4

Data collection and analysis- 7

Pumpage sampling-_- 7

Sampling strategy-_- 7

Instrument testing and measurements- 8

Pumpage relationships-_- 14

Cropland mapping- 16

Reported data-- 19

Physical inventory-_-_ 19

Aerial photographs- 21

Aerial photographs and Landsat data combined-_-_-_-_-_- 22

Landsat data-- 22

Summary and conclusions-_- 23

1980 water-use plan-_- 26

References cited-_- 30

Supplemental information-_- 31

Pumpage data for irrigation sites-_-__- 31

Explanation of terms for tables 6-15 31

\section{ILLUSTRATIONS}

Figures 1-3. Maps showing:

1. Location of High Plains aquifer- 3

2. Location of 1979 pumpage sampling areas-________-_ 6

3. Location of four quadrants used for evaluation of climatic effects

4. Graph showing comparison of Vibration Time Totalizer time of operation with time of operation determined from electric meter sources-

5. Graph showing comparison of Vibration Time Totalizer time of operation with time of operation determined from non-electric meter sources-

6-8. Maps showing:

6. Locations where cropland mapping techniques were tested during 1979---

7. Location of 1980 pumpage monitoring areas-

8. Number and location of Landsat scenes required to provide coverage of the High Plains aquifer-3
6

9. Diagram illustrating site-location numbering system for tables 6-13- 
TABLES

Page

Table 1. Area of High Plains aquifer and estimated acreage irrigated by ground water, 1977-

2. Summary of water applied in all areas separated by crop

3. Sume and irrigation system-ary of water applied in the northern area separated by quadrant and irrigation system-

4. Normal precipitation and actual precipitation from November 1978 through October 1979 in the test areas----

5. Irrigated cropland estimates for Phillips and Yuma Counties, Colorado, 1978-

6. Pumpage data for irrigation sites in Kit Carson County, Colorado--

7. Pumpage data for irrigation sites in Phillips County,

8. Pumpage data for irrigation sites in Yuma County, Colorado--

9. Pumpage data for irrigation sites in Cheyenne County, Kansas--

10. Pumpage data for irrigation sites in Sherman County, Kansas-_- County

11. Pumpage data for irrigation sites in Chase County, Nebraska--

12. Pumpage data for irrigation sites in Dundy County, Nebraska-

13. Pumpage data for irrigation sites in Perkins County, Nebraska--

14. Pumpage data for irrigation sites in Hockley County, Texas- 107

15. Pumpage data for irrigation sites in Lamb County, Texas---- 113 
CONVERSION TABLE

Multiply inch/pound units

inch

mile

acre

acre-foot

square mile

horsepower

cubic foot per

minute

gallon per minute
By

$$
25.40
$$

1.609

0.4047

1,233

2.590

745.7

0.4719

0.06309
To obtain metric unit

millimeter

kilometer

square hectometer

cubic meter

square kilometer joule per second

liter per second

liter per second 
EVALUATING METHODS FOR DETERMINING WATER USE IN THE HIGH PLAINS

IN PARTS OF COLORADO, KANSAS, NEBRASKA, NEW MEXICO, OKLAHOMA, SOUTH DAKOTA, TEXAS, AND WYOMING; 1979

By Frederick J. Heimes and Richard R. Luckey

\section{ABSTRACT}

The volume and areal distribution of ground-water pumpage (withdrawals) for irrigation during 1980 are required for the High Plains Regional AquiferSystem Analysis. During 1979, approaches and instrumentation that might be suitable for application to 1980 water-use determinations were tested. Pumpage was sampled by monitoring time of operation and discharge of irrigation wells during the growing season. The total volume pumped during the irrigation season was compared to the crop and acreage irrigated. This comparison provided a means of extending sampled pumpage information to unmonitored areas using irrigated-cropland maps.

A transient-time flowmeter proved to be reliable for providing discharge data and a vibration-sensitive timing device for providing time of operation information. Statistical analysis of comparisons between pumpage and irrigated cropland indicated that significant differences existed in the amounts of water applied between flood- and sprinkler-irrigation systems. However, statistical analyses of differences in amounts of water applied for various crop types and for selected climatic factors were inconclusive.

A variety of approaches were tested to develop the irrigated-cropland maps needed to extend sampled-pumpage data. Of the methods tested, only Landsat imagery proved to be cost effective for application to an area as large as the High Plains. The results obtained during the 1979 evaluation of instrumentation and pumpage-sampling approaches have been used to formulate a strategy for monitoring irrigation-water use in the High Plains during 1980. 


\section{INTRODUCTION}

The U.S. Geological Survey began a 5-year study of the High Plains regional aquifer during 1978 to provide the hydrologic information needed for the development of computer models to evaluate the aquifer's response to ground-water management alternatives. The plan of study for the High Plains Regional Aquifer-System Analysis is described by Weeks (1978). This report describes the approaches and instrumentation that were tested for sampling ground-water pumpage (withdrawa1) in parts of the High Plains during 1979. These tests were conducted to define the most suitable techniques for determining ground-water use for irrigation in the High Plains region during 1980 .

Collection and analysis of data for this effort required the cooperation of U.S. Geological Survey, National Aeronautics and Space Administration, State, and local employees, and individual irrigators. Without their support this study could not have been conducted.

\section{High Plains Description.}

The High Plains aquifer underlies an area of approximately 177,000 square miles, including parts of Colorado, Kansas, Nebraska, New Mexico, Oklahoma, South Dakota, Texas, and Wyoming (fig. 1). This aquifer, which is composed of saturated gravel, sand, silt and clay, is the only source of irrigation water for most of the area. The flat to gently rolling terrain that characterizes the High Plains region is extensively cultivated and the area has undergone rapid development for irrigation in recent years.

Mean annual precipitation in the region ranges from about 14 to 30 inches with about 75 percent falling during the growing season. However, persistent winds and hot summer temperatures cause large rates of evaporation in the High Plains. Class A pan evaporation ranges from about 60 inches per year in northern Nebraska to 100 inches per year in western Texas. Because of the small amounts of precipitation and the large evaporative demand, little precipitation is available to recharge the ground-water system. A detailed discussion of the physiography and climate of the High Plains region is contained in Gutentag and Weeks, 1980.

\section{Water Use}

Prior to 1930, agriculture on the High Plains consisted primarily of dry-land farming and cattle grazing. Ground-water irrigation developed rapid1y after the "Dust Bowl" days with the availability of inexpensive energy and technical advances in drilling and pumping techniques. During 1952, about 26,000 wells were used to pump 3.0 million acre-feet of water on 2.7 million acres of land (Lohman, 1953) and by 1977, 168,000 wells were used to pump 30 million acre-feet of water on 16 million acres of land (Gutentag and Weeks, 


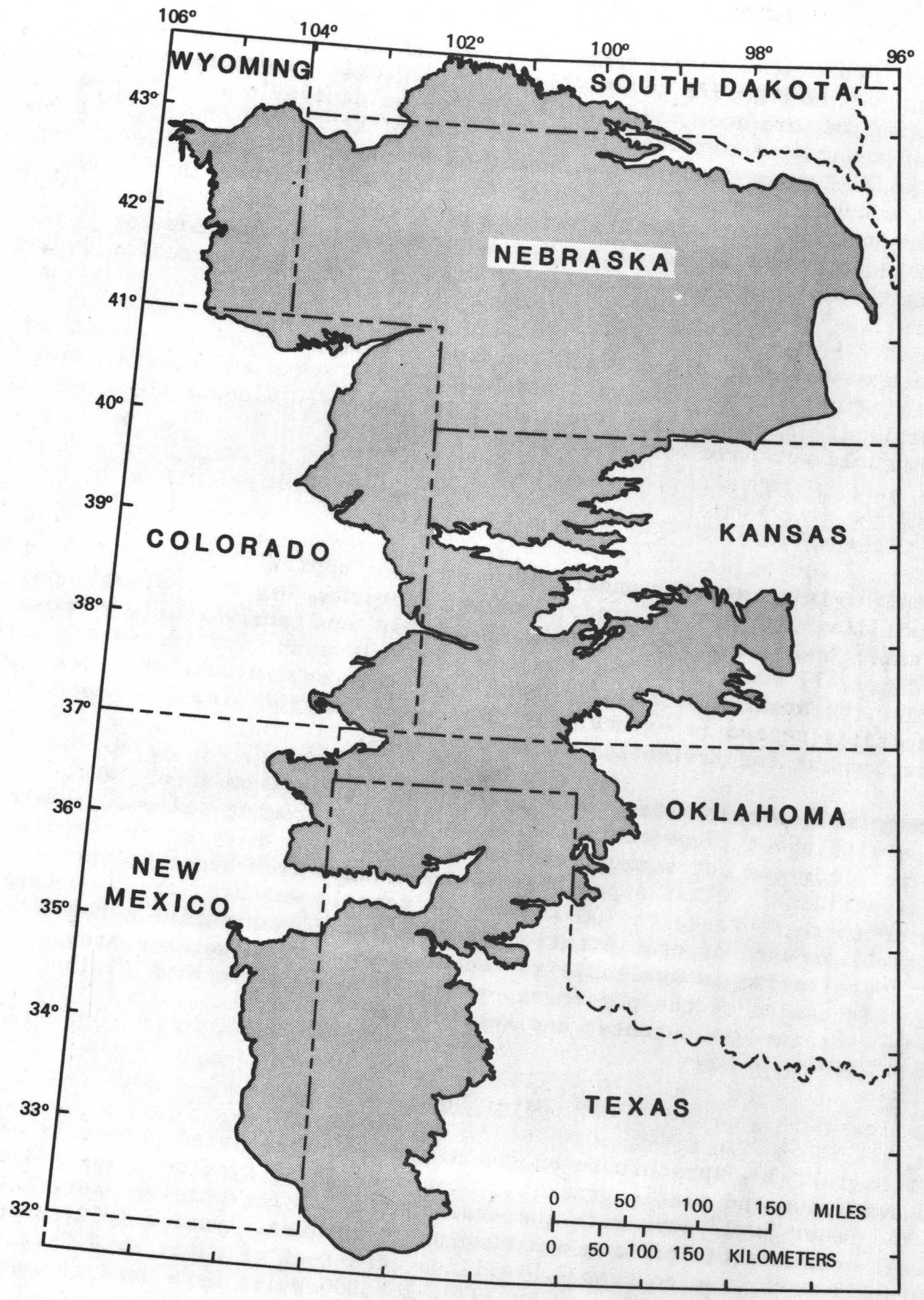

Figure 1.-Location of High Plains aquifer (shaded). 
1980). Estimates of acreage and the number of wells by States in the High Plains during 1977 are summarized in table 1. The acreage currently irrigated by the High Plains aquifer represents approximately 23 percent of the irrigated land within the United States. During 1977, about 35 percent of the area of . the High Plains was cropland, and about 14 percent of the area was irrigated.

Based on 1977 crop statistics compiled by the U.S. Department of Agriculture, about 15 percent of the corn, 70 percent of the grain sorghum, and 23 percent of the upland cotton produced in the United States were grown in the High Plains States. Receipts from irrigated crops and related beef cattle production in this region exceed 12 billion dollars annually according to recent estimates (Grubb, 1978). Consequent1y, significant changes in the amount or distribution of irrigated cropland could affect the economy of the High Plains region dramatically.

Extensive development of the ground-water system for irrigation, combined with the small rate of recharge has resulted in large water-table declines in many areas of the High Plains. Various approaches have been suggested to control the declining water levels in the High Plains region. These approaches include: Increased use of conservation techniques, importation of water to augment existing supplies, and artificially increasing available water within the region through weather modification or changes in land-management techniques. To evaluate these or other management approaches, reliable and accurate water-use information is needed.

Sampled pumpage cannot be extrapolated directly to unmeasured wells and it is impossible to monitor ground-water pumpage at each of the approximately 170,000 irrigation wells located in the High Plains region. However, ground-water pumpage for irrigation is related to crop acreage irrigated. By establishing the relationships between irrigated acreage and ground-water pumpage, estimates of pumpage could be compiled for large areas using cropland maps combined with sampled pumpage information.

\section{APPROACH}

Two test areas (fig. 2) were selected to develop methods and test instrumentation for determining ground-water use for irrigation in the High Plains. The eight county test area located in the northern High Plains region included three counties (Phillips, Yuma, and Kit Carson) in Colorado, two counties (Cheyenne and Sherman) in Kansas, and three counties (Chase, Perkins, and Dundy) in Nebraska. The two-county test area located in the southern High Plains included Hockley and Lamb Counties in Texas. The approach for determining water use included: (1) Developing a statistical approach to sample ground-water pumpage for irrigation, (2) testing instrumentation, (3) developing suitable procedures for measuring the annual volume of ground water pumped from selected irrigation wells, (4) developing relationships between the annual volume of ground water pumped and the acreage irrigated, and (5) determining a suitable approach to mapping irrigated cropland for the entire High Plains region. 
Table 1.--Area of High Plains aquifer and estimated acreage irrigated by ground water, 1977 (from Gutentag and Weeks, 1980)

\begin{tabular}{|c|c|c|c|c|c|}
\hline \multirow[t]{2}{*}{ State } & \multicolumn{2}{|c|}{$\begin{array}{c}\text { Area of State within } \\
\text { High Plains }\end{array}$} & \multirow{2}{*}{$\begin{array}{c}\text { Area of High Plains } \\
\text { within State } \\
\text { (percent) }\end{array}$} & \multirow{2}{*}{$\begin{array}{l}\text { Estimated } \\
\text { irrigated } \\
\text { acres }^{l}\end{array}$} & \multirow{2}{*}{$\begin{array}{l}\text { Estimated } \\
\text { number } \\
\text { of wells }\end{array}$} \\
\hline & (square miles) & (percent) & & & \\
\hline Colorado-_-__-_ & 14,870 & 14 & 8 & 585,000 & 4,300 \\
\hline Kansas-_-__- & 31,050 & 38 & 18 & $3,250,000$ & 23,000 \\
\hline Nebraska-_- & 64,400 & 83 & 36 & $5,560,000$ & 59,300 \\
\hline New Mexico-_-_- & 9,710 & 8 & 6 & 480,000 & 6,000 \\
\hline Oklahoma-_____ & 7,350 & 11 & 4 & 225,000 & 2,200 \\
\hline South Dakota- & 5,290 & 7 & 3 & 20,000 & 160 \\
\hline Texas- & 36,080 & 13 & 20 & $6,000,000$ & 72,000 \\
\hline Wyoming- & 8,190 & 8 & 5 & 90,000 & 1,140 \\
\hline TOTALS- & 176,940 & - & 100 & $16,210,000$ & 168,000 \\
\hline
\end{tabular}

${ }^{1}$ The data were obtained from State and Federal agencies and adjusted to include only the areas irrigated by ground water. 


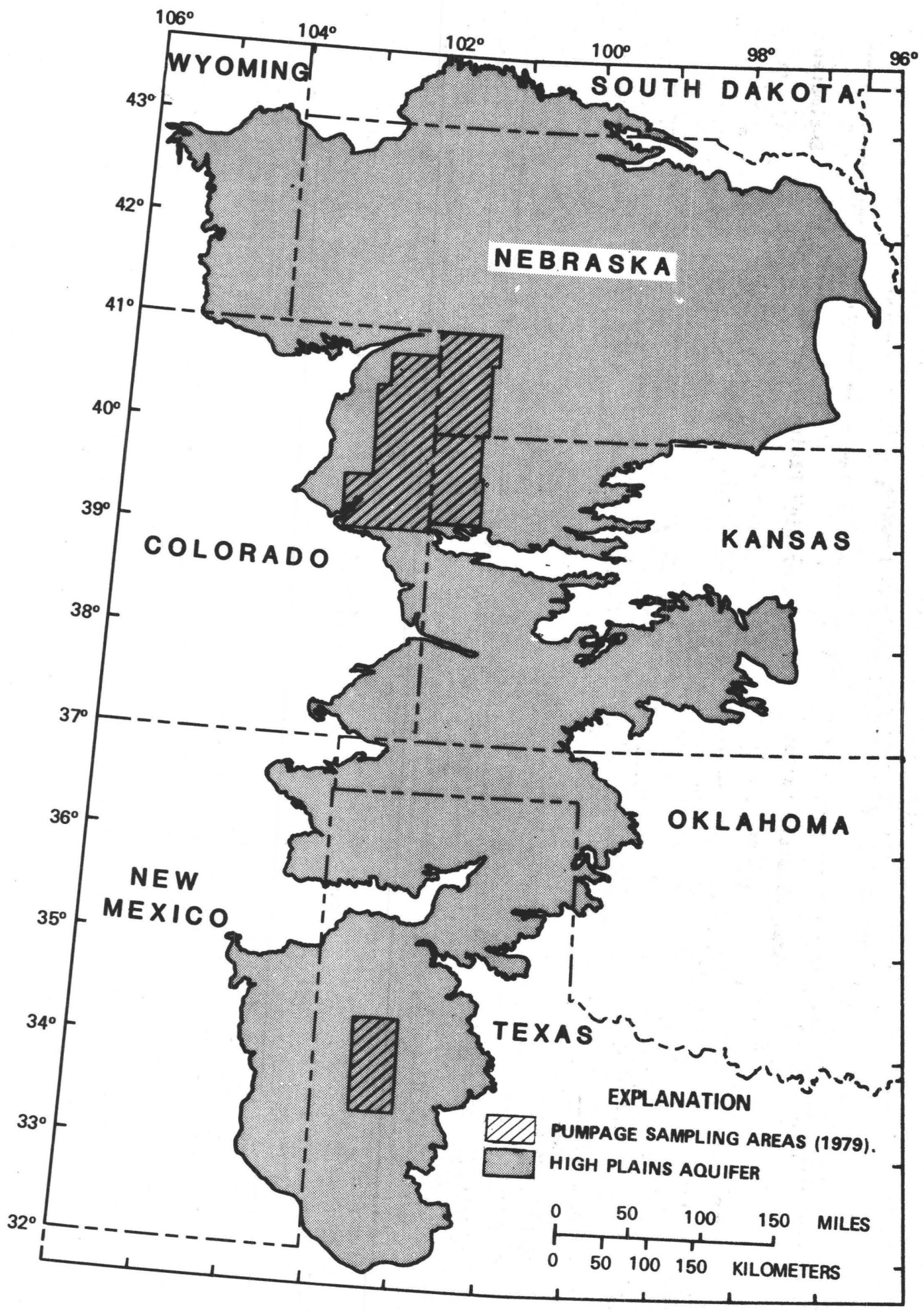

Figure 2.- Location of 1979 pumpage sampling areas. 
The annual volume of ground water pumped was measured at about 250 irrigation wells. The annual volume pumped was computed by multiplying the pumping rate by the total time of pumping. Several types of portable flowmeters for measuring pumping rate, and one timing device to monitor total time of pumping, were tested for suitability and accuracy. The depth of water applied (volume pumped divided by the acreage irrigated) was calculated for various crop types, irrigation system types, and climatic zones to determine which of these factors may be important in estimating and extending sampled pumpage to other areas of the High Plains.

In order to provide maps for extending sampled pumpage data, various approaches for mapping irrigated cropland were evaluated in the northern eight-county test area. The five mapping approaches tested included:

(1) Reported data, (2) physical inventory, (3) large scale aerial photography, (4) a combination of small scale aerial photography and Landsat images, and

(5) Landsat imagery alone.

These five techniques were selected because each potentially could provide maps depicting the areal distribution of irrigated cropland on a seasonal basis. Each approach was evaluated for relative accuracy and suitability for application to large areas.

\section{DATA COLLECTION AND ANALYSIS}

\section{Pumpage Sampling}

Pumpage-data collection and analysis consisted of three phases:

(1) Developing a sampling strategy and selecting sites in the two test areas;

(2) conducting measurements and testing instruments for determining total time of operation, discharge, and the crop type and acreage being irrigated at each site; and (3) statistical analysis of pumpage data to determine significant relationships.

\section{Sampling Strategy}

A stratified random sampling procedure was used to select the 250 , sites to be monitored during the 1979 irrigation season. Sampling sites were defined as individual irrigation wells or groups of irrigation wells that were combined to form a single irrigation unit. Two hundred sampling sites were selected in the northern test area and 50 sites were selected in the southern test area using lists of all irrigation wells located within each area. Sites were stratified by crops to investigate potential differences in pumpage between crop types, and by area to investigate climatic effects on pumpage.

Crop-type stratifications included the major irrigated crops in the two test areas: Corn, alfalfa, and mixed crops in the northern test area and cotton in the southern test area. The number of sites selected for each crop was based on the relative proportion of that crop to other irrigated crops in 
the area. One hundred forty sites that irrigated corn, 40 sites that irrigated alfalfa, and 20 sites that irrigated mixed crops were defined in the northern test area. Because of the small size of alfalfa fields in the area, it was difficult to locate sites that irrigated solely alfalfa; consequently, during the final selection process some sites designated for alfalfa actually ended up to be mixed crop sites. All 50 sites in the southern test area irrigated cotton.

The northern test area also was subdivided (stratified) into four quadrants (fig. 3), with each containing 50 sampling sites. The quadrants were selected to investigate the effects of variations in precipitation and potential evapotranspiration on pumpage.

\section{Instrument Testing and Measurements}

An instrument was developed for the National Water-Use Data program of the U.S. Geological Survey to measure the time of operation of irrigation systems. This device is referred to as a Vibration Time Totalizer (VTT); it is about 1 1/2 inches X 1 inch X 3/4 inch and is attached to the pump base or the discharge pipe. The VTT records the elapsed time that it is subjected to vibration. Virtually all of the irrigation systems in use in the High Plains region generate enough vibration while operating to activate the VTT units.

A11 of the VTT units used during the 1979 irrigation season were handmade and thus subject to large variations in quality. Part of the testing process during 1979 was to determine the accuracy and durability of the VTT units. One VTT was placed at each of the sites that were used to sample pumpage. The VTT units were placed on the pump or discharge pipe in an area that would provide sufficient vibration while affording some protection from heat and water.

In order to evaluate the reliability and accuracy of the VTT units, an independent determination of time of operation also was attempted at many of the sampling sites. The most common and reliable alternative source of time of operation was the energy meters. Electric meters provided the most reliable alternative measure of time of operation for the irrigation wells in the High Plains. Gas meters also provide a measure of time of operation, but the reliability of gas meter calculations is much less than for electric meters because the rate of gas consumption by a pumping plant is much more variable than the rate of electric consumption.

Other independent methods of determining the total time of operation, including engine-hour meters and records kept by irrigators, also were used to record time of operation when possible. Engine-hour meters generally are accurate but quite susceptible to mechanical problems. The accuracy of records kept by irrigators need to be determined on an individual basis. 


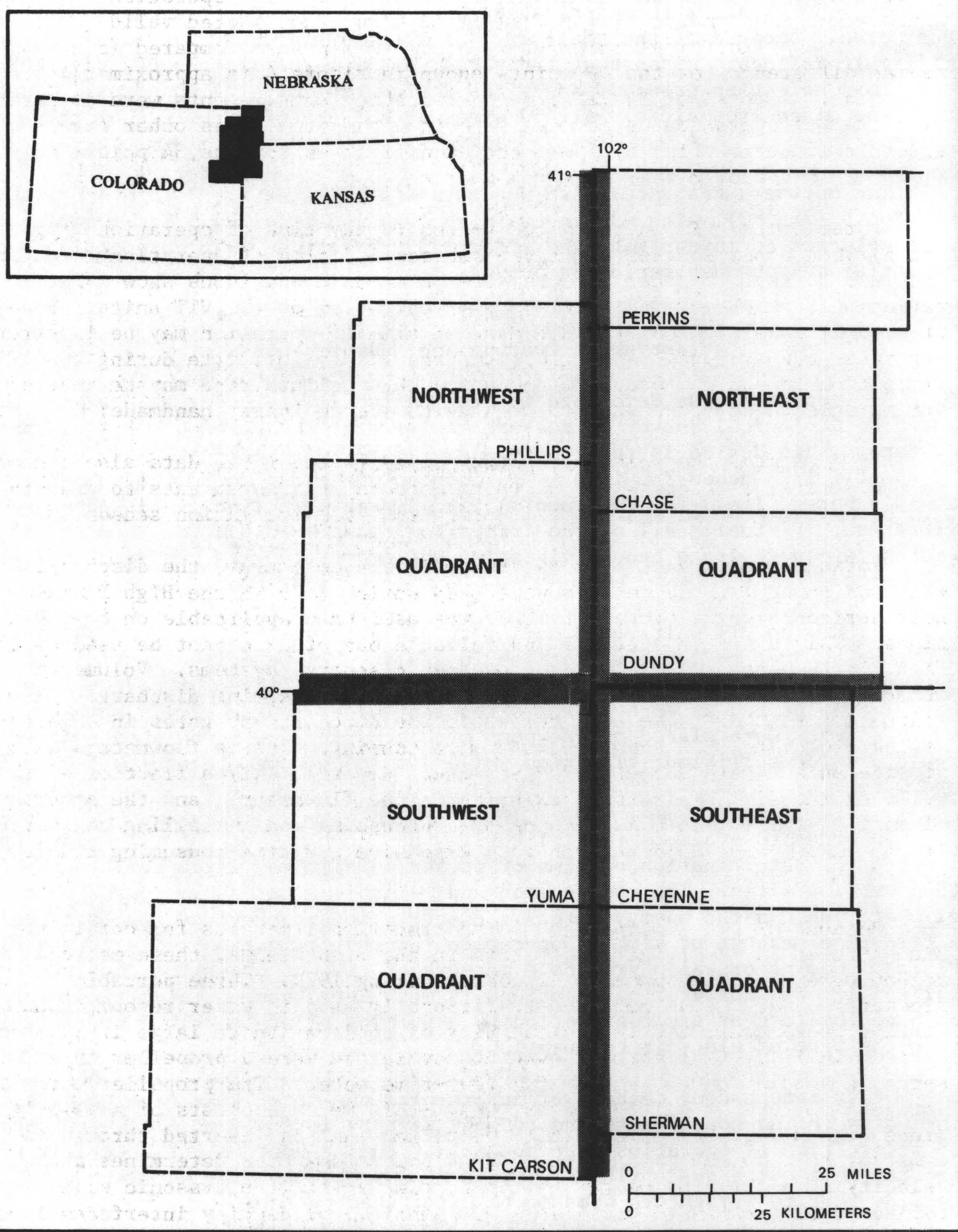

Figure 3.--Location of four quadrants used for evaluation of climate efforts. 
At the end of the 1979 irrigation season, comparisons of VTT time of operation were made with independently acquired time of operation for all sites where independent time determinations were considered valid. A total of $40 \mathrm{VTT}$ and electrical meter times of operation were compared "(fig. 4). The median difference for the 40 points shown in figure 4 is approximately 5 percent. A total of 34 VTT time-of-operation measurements were compared with time of operation determined from independent sources other than electrical meters (fig. 5). The median difference for the 34 points shown in figure 5 is approximately 13 percent.

Three of the VTT units failed to record any time of operation (figs. 4 and 5) when other sources indicated substantial time of operation (greater than 250 hours). Another six time-of-operation comparisons show large variations, which may indicate improper operation of the VTT units. However, it also is possible that the independent time of operation may be in error for these comparisons. An estimate of the VTT failure rate during the 1979 irrigation season was about 10 percent. This failure rate may be reduced during subsequent years when the VTT units are no longer handmade.

To determine the volume of water pumped by the well, data also are needed on the discharge of the well. Several methods and instruments to measure discharge of the well were tested during the 1979 irrigation season.

Various traditional methods are available to measure the discharge of a well and some of these methods were used during 1979 in the High Plains. The Hoff horizontal-axis propeller meter was used when applicable on open discharge pipes. This meter is accurate and reliable but often cannot be used on the High Plains because there are so few open discharge systems. Volumetric methods also are simple and accurate methods of measuring discharge. The

inline flowmeters would be both very expensive and time consuming beyond the resources of this project.

taining

ethods

rrigation

type

meter is

velocity

ough an

the

waves

aces in the ire access
Because of the limitations of the traditional methods for ob discharge on large irrigation systems in the High Plains, these m seldom were used for sampling discharge during 1979. Three porta flowmeters, which have not been traditionally used in water resou studies, were evaluated for suitability of application to large i systems in 1979. The three flowmeters evaluated were a propeller meter, a Doppler meter, and a transient-time meter. The propelle designed for use in gated-pipe systems. This meter consists of a probe with horizontal axis turbine propeller that is inserted thr existing hole in the gated pipe. The Doppler flowmeter determine velocity of a fluid by measuring the Doppler shift of ultrasonic reflected off entrained particles, air bubbles, or density interf fluid stream. This meter has the advantage that it does not requ 


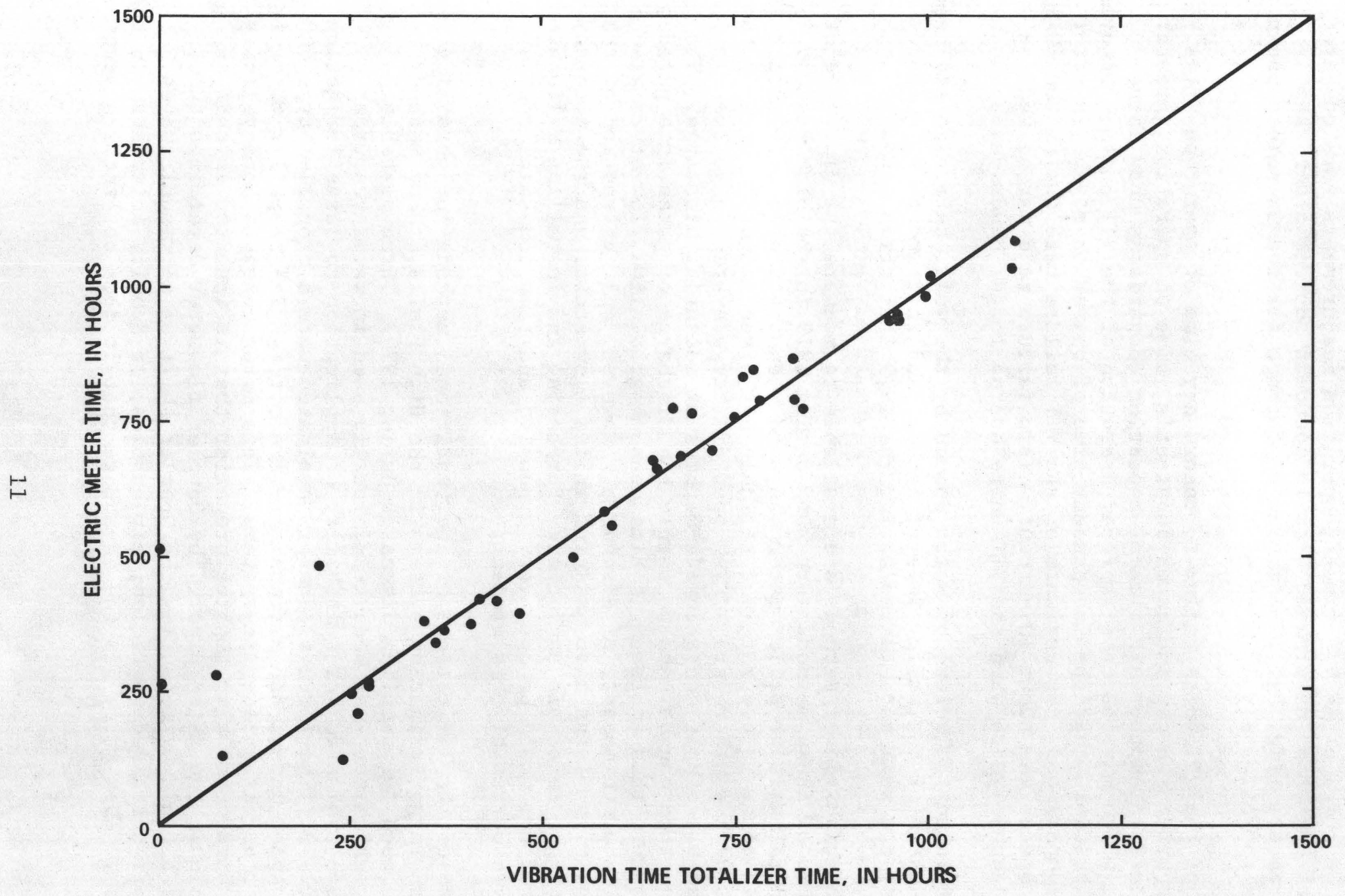

Figure 4.--Comparison of Vibration Time Totalizer time of operation with time of operation determined from electric meter sources. 


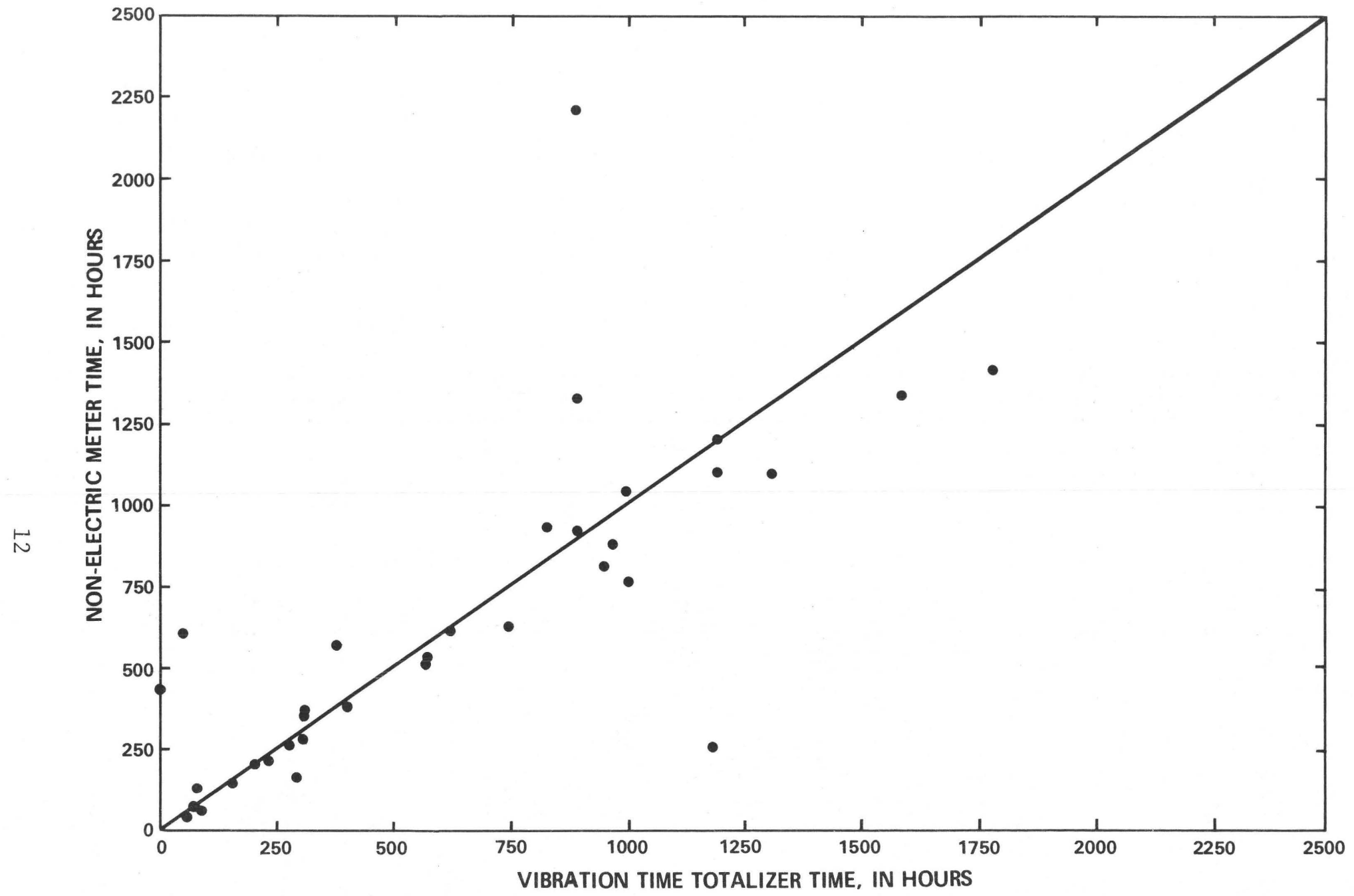

Figure 5.-Comparison of Vibration Time Totalizer time of operation with time of operation determined from non-electric meter sources. 
to the fluid stream to obtain a measurement of discharge. The transienttime flowmeter, like the Doppler flowmeter, uses ultrasonic waves to measure the fluid flow and also does not require access to the fluid stream. However, the principal used in this type of meter does not require entrained particles, air bubbles or density interfaces to operate properly.

Detailed testing of all three instruments was done under both laboratory and field conditions during 1979. All three instruments were tested on a variety of pipe sizes and materials commonly found in irrigation systems on the High Plains. A brief description of the results of this testing is presented here; detailed information is reported by Luckey, Heimes, and Gaggiani (1980).

The propeller meter was found to be a rugged instrument but is difficult to use on systems that are under pressure. It requires a considerable amount of time and skill to operate effectively, as well as a willingness on the part of the operator to get wet and muddy. This meter also is very sensitive to particulate matter in the water and must be cleaned and lubricated frequently. The propeller meter gave consistently smaller than actual velocity readings both in field and laboratory tests.

The Doppler meter was the easiest meter to use under field conditions. A velocity reading generally could be obtained on aluminum pipe of as much as 8 inches in diameter; however, velocity readings seldom could be obtained on 6-inch or larger diameter steel pipe. Even when the instrument appeared to be operating properly, velocity readings frequently were not reliable.

The transient-time meter was not designed to be a portable field instrument and was somewhat awkward to use under field conditions. It is fairly bulky and requires an external 110 volt a-c electrical current. However, with practice, a measurement using the transient-time meter can be made in 15 to 30 minutes. The transient-time meter was tested and calibrated on 17 different kinds and sizes of pipe under laboratory conditions and was found to give extremely reliable results. A separate calibration is needed for each kind and size of pipe encountered. This meter was the only one of the three meters tested that consistently provided reliable results in measuring discharge on the wide variety of pipes that are in use in High Plains irrigation systems.

To compute the depth of water applied at each site, the annual volume of water pumped (average discharge multiplied by time of operation) is divided by the irrigated acreage. Irrigated crop type and acreage at each sampling site generally were determined by one of the following methods: Physical measurement of field dimensions, reported and verified crop acreage information obtained from local Agricultural Stabilization and Conservation Service offices, or information provided by individual irrigators. 
The data collected during the 1979 irrigation season are tabulated in the supplemental section of this report. This supplement lists all of the discharge and time of operation measurements that were collected at the sampling sites, even though many of these measurements were made using instruments or techniques that later proved to be unreliable. These measurements are shown, not because they are thought to represent accurate values of discharge and time, but because these measurements document the reliability of the instruments and techniques that were evaluated. The data shown in this supplement are experimental data and as such, any particular measurement may be subject to large errors. The 1979 pumpage-sampling program was designed to test techniques and instrumentation, and as the data in the supplement clearly indicate, some of the techniques and instruments tested were not appropriate.

Although some of the individual measurements listed in the supplement may have errors associated with them, aggregate information is of sufficient quality to determine suitable approaches and instrumentation for collecting water-use data on the entire High Plains during 1980.

The total volume of water pumped at each site and the depth of water applied to the field were calculated where adequate discharge, time of operation, and crop acreage data were available. These calculations were made using the best estimates of discharge and total time of operation. The data were analyzed statistically with summaries by crop type, irrigation system type, and geographical area. No statistical entries were made in the tables when the total number of data points within any group was less than 10 .

A comparison of the depth of water applied by crop and type of irrigation system is shown in table 2. The information on cotton is from the southern test area; all other information is from the northern test area.

The mean application is the arithmetic mean of the application of all the data points in a group. The mean application multiplied by the total acreage in a group gives an estimate of the total pumpage for a group. The standard deviation is a measure of the dispersion of the data about the mean. The relatively large standard deviation associated with most of the groups indicates that there is considerable scatter in the individual data points. The standard error of the mean is a measure of the reliability of the sample mean as an estimator of the true population mean. There is about a 95 percent chance that the true mean is within two standard errors of the calculated mean. The sample size is the total number of sample sites within each category that were included in this analysis.

Analysis of the information in table 2 indicates that sprinkler systems tend to apply less water than flood systems. This hypothesis was found to be statistically significant at the 95 percent confidence level. The difference between water applied to corn and alfalfa was found to be significant at the 95 percent confidence level for sprinkler systems but was 
Table 2.--Sumary of water applied in all areas separated by crop type and irrigation system

[Data on cotton are from the southern area; all other data are from the northern area]

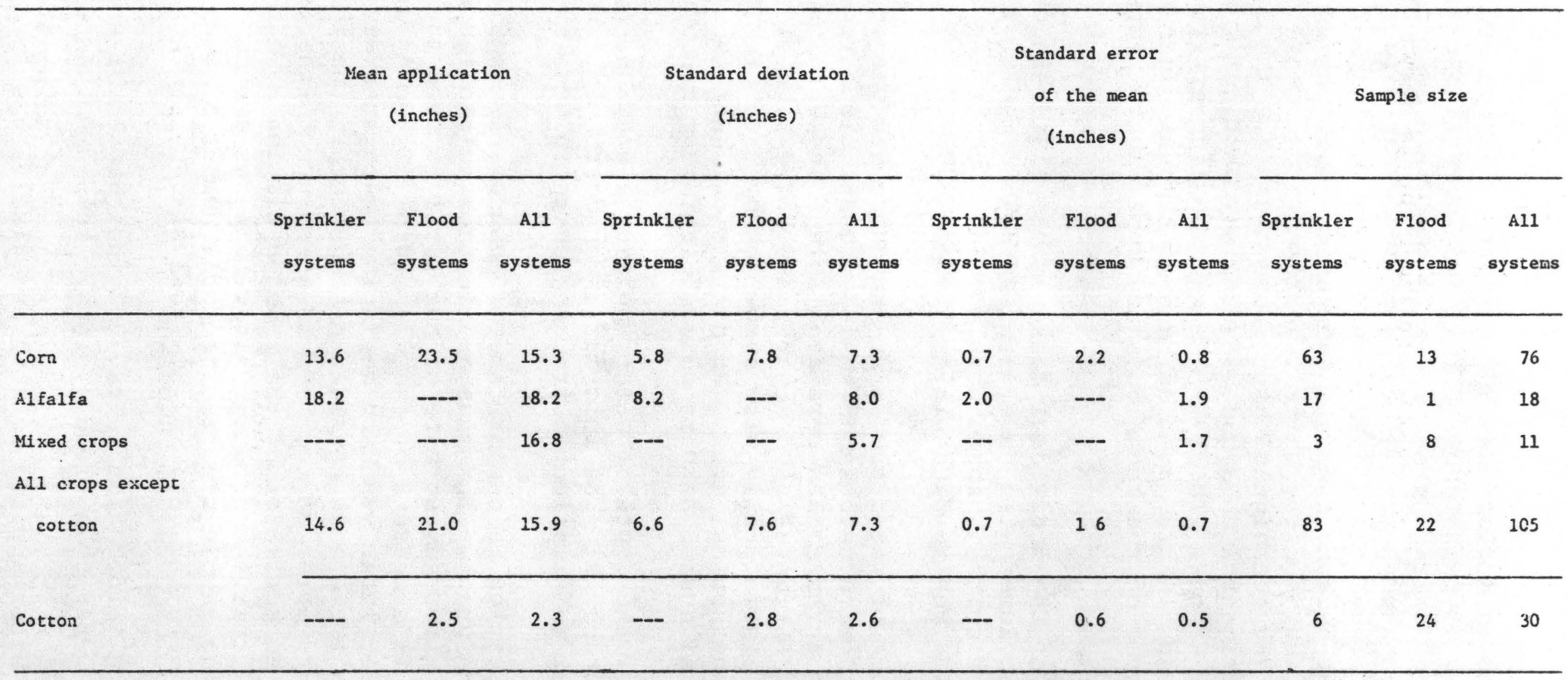


not statistically significant when both types of systems were combined in the analysis. This lack of significance may be due to the increased variability when data from the two types of systems are combined.

A comparison of depth of water applied in the northern test area separated by type of irrigation system and quadrant is shown in table 3 . The difference in the depth of water applied between irrigation system types is statistically significant at the 95 percent confidence level for each of the four comparisons where data are shown from both sprinkler irrigation systems and flood irrigation systems.

The standard deviations shown in table 3 are smaller for sprinkler irrigation systems than they are for flood irrigation systems. This would indicate that there is less variability in water use among individual sprinkler irrigation systems than there is among individual flood irrigation systems. However, the data do not prove such a hypothesis at the 95 percent confidence leve1.

There is no significant difference (95 percent confidence leve1) between the amount of water applied between quadrants or pairs of quadrants. Such a difference might be expected because of the difference in precipitation among the quadrants (table 4). However, there are obviously many factors other than total annual precipitation that govern the amount of irrigation water applied.

The data collected during 1979 could be used to calculate total water use for irrigation in the northern area. Because data were not collected by system type or crop type on the total irrigated acreage in the test area, the mean application for all crops and systems would have to be used to calculate total water use. This mean application (15.9 inches) has a standard error of 0.7 inch. The 95 percent confidence limits on the mean application for all crops and systems in the northern areas is $15.9 \pm 1.4$ inches.

However, there may be other measuring errors that exist that are not part of this calculated sampling error. If total irrigation water use was calculated for the northern test area, all of these errors would need to be quantified to determine the accuracy of these calculations.

\section{Cropland Mapping}

The mapping approaches evaluated for suitability in depicting the areal distribution of irrigated cropland were conducted concurrently with pumpage sampling in the eight-county northern test area.

Reported data from the Agricultural Stabilization and Conservation Service (ASCS) were used to map crop and rangeland in Kit Carson, Phillips, and Yuma Counties in Colorado. Physical inventory was used to map irrigated cropland in Cheyenne and Sherman Counties in Kansas. Color infrared aerial photographs at a scale of 1:24,000 were visually interpreted to map cropland along transects in Phillips and Yuma Counties in Colorado, Chase and Dundy Counties in Nebraska, and Cheyenne County in Kansas. Color infrared aerial photographs 
Table 3.--Sumary of water applied in the northern area separated by quadrant and irrigation system

IData from all crops are aggregated]

\begin{tabular}{|c|c|c|c|c|c|c|c|c|c|c|c|c|}
\hline \multirow[t]{2}{*}{ Quadrant } & \multicolumn{3}{|c|}{$\begin{array}{l}\text { Mean application } \\
\text { (Inches) }\end{array}$} & \multicolumn{3}{|c|}{$\begin{array}{c}\text { Standard deviation } \\
\text { (inches) }\end{array}$} & \multicolumn{3}{|c|}{$\begin{array}{c}\text { Standard error } \\
\text { of the mean } \\
\text { (inches) }\end{array}$} & \multicolumn{3}{|c|}{ Sample size } \\
\hline & $\begin{array}{l}\text { Sprinikler } \\
\text { systems }\end{array}$ & $\begin{array}{c}\text { Flood } \\
\text { systems }\end{array}$ & $\begin{array}{c}\text { A11 } \\
\text { systems }\end{array}$ & $\begin{array}{l}\text { Sprinkler } \\
\text { systems }\end{array}$ & $\begin{array}{c}\text { Flood } \\
\text { systems }\end{array}$ & $\begin{array}{c}\text { All } \\
\text { systems }\end{array}$ & $\begin{array}{l}\text { Sprinkler } \\
\text { systems }\end{array}$ & $\begin{array}{c}\text { Flood } \\
\text { systems }\end{array}$ & $\begin{array}{c}\text { Al1 } \\
\text { systems }\end{array}$ & $\begin{array}{l}\text { Sprinkler } \\
\text { systems }\end{array}$ & $\begin{array}{l}\text { Flood } \\
\text { systems }\end{array}$ & $\begin{array}{c}\text { All } \\
\text { systems }\end{array}$ \\
\hline \multicolumn{13}{|l|}{ Northeast } \\
\hline quadrant & 13.5 & $\cdots$ & 14.9 & 6.0 & $\cdots$ & 7.2 & 1.1 & $\cdots$ & 1.3 & 28 & 3 & 31 \\
\hline \multicolumn{13}{|l|}{ Northwest } \\
\hline quadrant & 16.4 & -- & 16.8 & 7.7 & $-\cdots$ & 8.0 & 1.3 & $\cdots$ & 1.3 & 35 & 1 & 36 \\
\hline \multicolumn{13}{|l|}{ Southeast } \\
\hline quadrant & 12.5 & 18.4 & 15.7 & 3.3 & 5.8 & 5.6 & 1.0 & 1.6 & 1.2 & 11 & 13 & 24 \\
\hline \multicolumn{13}{|l|}{ Southwest } \\
\hline quadrant & $\cdots$ & -- & 16.5 & - & - & 8.0 & -- & - & 2.2 & 9 & 5 & 14 \\
\hline \multicolumn{13}{|l|}{ Eastern } \\
\hline quadrants & 13.3 & 20.1 & 15.2 & 5.4 & 6.7 & 6.6 & 0.9 & 1.7 & 0.9 & 39 & 16 & 55 \\
\hline \multicolumn{13}{|l|}{ Western } \\
\hline quacrants & 15.8 & - & 16.7 & 7.3 & - & 8.0 & 1.1 & $\cdots$ & 1.1 & 44 & 6 & 50 \\
\hline \multicolumn{13}{|l|}{ Nor thern } \\
\hline quadrants & 15.1 & -- & 15.9 & 7.2 & - & 7.7 & 0.9 & $\cdots$ & 0.9 & 63 & 4 & 67 \\
\hline \multicolumn{13}{|l|}{ Southern } \\
\hline quadrants & 12.9 & 19.5 & 16.0 & 4.0 & 7.3 & 6.6 & 0.9 & 1.7 & 1.1 & 20 & 18 & 38 \\
\hline All quadrants & 14.6 & 21.0 & 15.9 & 6.6 & 7.6 & 7.3 & 0.7 & 1.6 & 0.7 & 83 & 22 & 105 \\
\hline
\end{tabular}


Tabie 4.--Normal precipitation and actual precipitation from

November 1978 through October 1979 in the test areas

\begin{tabular}{|c|c|c|c|c|c|}
\hline \multirow[b]{2}{*}{ Station name } & \multirow{2}{*}{$\begin{array}{c}\text { County and } \\
\text { State }\end{array}$} & \multirow{2}{*}{$\begin{array}{l}\text { Normal annual } \\
\text { precipitation } \\
\text { (inches) }\end{array}$} & \multirow[b]{2}{*}{ Quadrant } & \multicolumn{2}{|c|}{$\begin{array}{c}\text { November } 1978 \text { through } \\
\text { October } 1979\end{array}$} \\
\hline & & & & $\begin{array}{c}\text { Total } \\
\text { precipitation } \\
\text { (inches) }\end{array}$ & $\begin{array}{c}\text { Deviation from } \\
\text { normal } \\
\text { (inches) }\end{array}$ \\
\hline Imperial & Chase Co., Nebr. & 19.15 & Northeast & 16.33 & -2.82 \\
\hline Holyoke & Phillips Co., Colo. & 18.40 & Northwest & 20.84 & +2.44 \\
\hline Goodland WSO AF & Sherman Co., Kans. & 16.65 & Southeast & 25.19 & +8.54 \\
\hline Burlington & Kit Carson Co., Colo. & 16.85 & Southwest & 18.44 & +1.59 \\
\hline Littlefield no. 2 & Lamb Co., Tex. & $18.0<$ & - - - - & 24.15 & +6.11 \\
\hline
\end{tabular}


at a scale of 1:80,000 were used in conjunction with 1979 Landsat images to interpret visually cropland categories in Chase, Dundy, and Perkins Counties in Nebraska. Visual and digital interpretation techniques were used to map irrigated cropland from 1978 Landsat imagery in Phillips and Yuma Counties in Colorado. The parts of the eight-county test area that were used for each mapping approach are shown in figure 6 .

\section{Reported Data}

Data reported by participants in the ASCS Feed Grain and Wheat program were used to map cropland in Kit Carson, Phillips, and Yuma Counties in Colorado. These data were obtained through a contractual arrangement with the ASCS county offices. The Feed Grain and Wheat program provides reimbursement for natural disasters that destroy crops, guarantees minimum target-price payments for crops, and makes available commodity loans to participants. Consequent1y, participation in the program varies from year to year in response to growing conditions and the economy. Approximately 3,400 square miles of land were mapped in the three counties. Map scale was about 7.5 inches to the mile, with one section (1 square mile) displayed on a single sheet of $81 / 2 \times 11$ inch paper. Maps were compiled primarily using reported crop acreages supplemented with 35-millimeter aerial photography acquired by the county offices for verification purposes.

ASCS offices verify about 25 percent of the reported information used for cropland mapping, so the reliability of cropland data obtained by this method is quite good. The ASCS data provided significant detail at an average cost of about $\$ 2.20$ per square mile. However, to apply this approach over an area as large as the High Plains would be quite difficult and costly. Computations of tabular data on crop acreage would increase the cost of this approach. The degree of variability in participation in the ASCS Feed Grain and Wheat program between counties from one year to the next could result in incomplete cropland maps for many areas. Additionally the logistics of contracting with each of the ASCS county offices in the High Plains to provide crop maps would be prohibitive at the present time.

Other forms of reported data are compiled by a variety of agencies. However, the majority of these data are aggregated as acreages by county and lack information on the areal distribution of cropland. Other agencies that regularly compile agricultural data and statistics include U.S. Department of Agriculture, Crop and Livestock reporting service, and Soil Conservation Service, U.S. Department of Commerce, Bureau of Census, and various State and local agencies.

\section{Physical Inventory}

Ground sampling to provide maps of irrigated cropland in Cheyenne and Sherman Counties, Kansas, was conducted by a private consulting firm. Crop types and areal extent of irrigated land were mapped at a scale of 1:250,000 


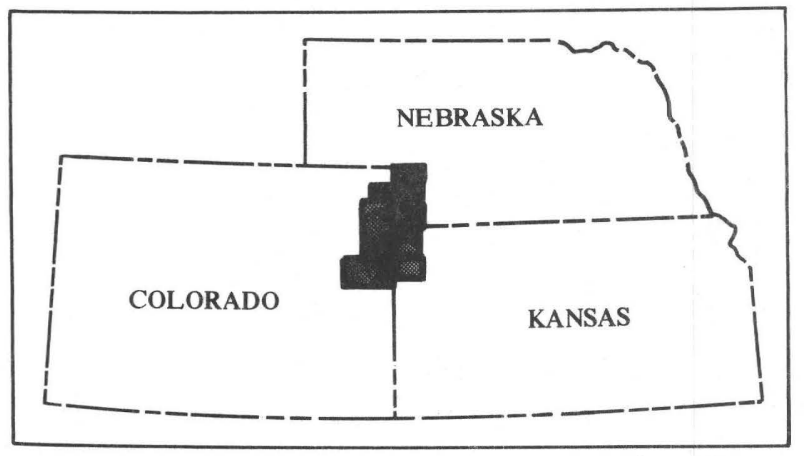

\section{EXPLANATION}

PHYSICAL INVENTORY

AGRICULTURAL STABILIZATION AND CONSERVATION SERVICE MAPPING

II, LANDSAT MAPPING

AERIAL PHOTOGRAPHS AND LANDSAT

AERIAL PHOTOGRAPHS
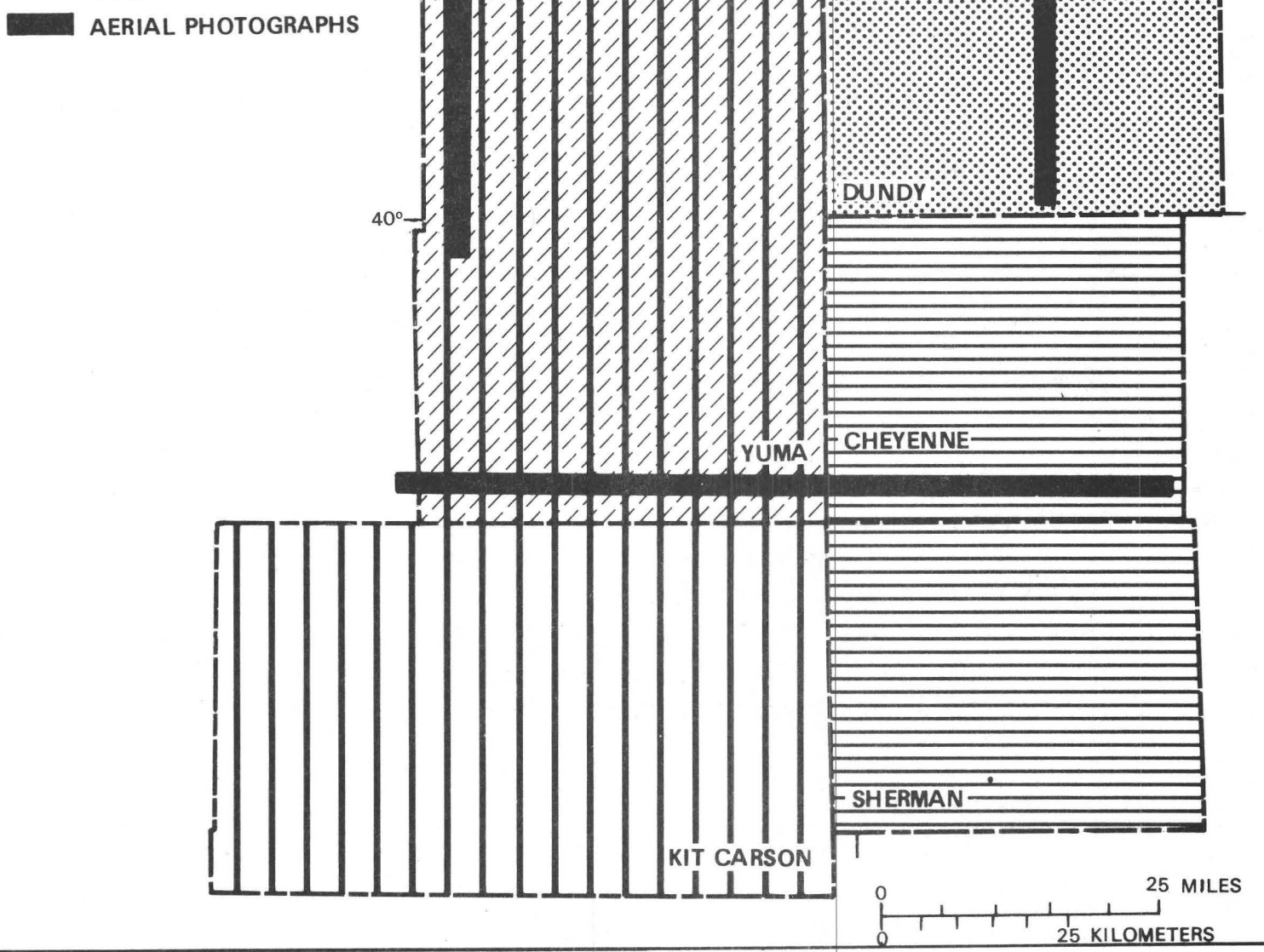

Figure 6.--Locations where cropland mapping techniques were tested during 1979. 
with a minimum resolution of 40 acres. Mapping was accomplished by actual field observation supplemented with limited landowner and ASCS information. Slightly more than 200 square miles of irrigated cropland were mapped in the two counties. Acreage figures were compiled, by county, for each of the irrigated crop types.

This approach to mapping irrigated cropland provided accurate results for crop-type determination and reasonably reliable delineation of field boundaries and acreages. Comparisons of these maps compiled from ground sampling with aerial photography in parts of Cheyenne County indicate that the crop-type determinations were accurate with a few exceptions. The areal delineation of fields was excellent along access roads but tended to be less accurate in areas away from access roads. However, at a scale of 1:250,000 these variations in areal delineation are not serious and tend to average out in providing a representative acreage figure for irrigated land aggregated by counties. This survey was conducted during June when most irrigated crops were in early stages of growth or emergence. More accurate results probably could have been obtained if mapping was done during July when crop types are more easily identified and before crop height obscures field boundaries. This approach is very time consuming and is the most expensive method tested. The $\$ 25.00$ per square mile cost makes it suitable on $1 y$ for application to localized areas requiring detailed crop information.

\section{Aerial Photographs}

Color infrared photographs at a scale of $1: 24,000$ were obtained for areas in Phillips and Yuma Counties in Colorado, Cheyenne County in Kansas, and Chase and Dundy Counties in Nebraska. The photographs included 190 miles of flight line covering approximately 640 square miles of land area. The photographs were obtained through an agreement with the U.S. Environmental Protection Agency and were interpreted visually by personnel of the U.S. Geological Survey at Ames Research Center, Mountain View, California.

Interpretation of these photographs provided excellent accuracy in mapping the areal distribution of cropland. Reliable crop-type mapping was obtained for irrigated cropland. Less accuracy was obtained in mapping dryland crops. Crop-type interpretations were compared with ASCS crop information for selected locations to verify the reliability of interpretations. The cost of the aerial photography was about $\$ 5.50$ per square mile of coverage. Cost of interpreting cropland from this photography and transferring it to 7.5 -minute quadrangles was estimated to be $\$ 1.00$ per square mile. The total cost for obtaining cropland maps at $1: 24,000$ scale by this method including photography and interpretation was estimated to be about $\$ 6.50$ per square mile. This cost is too expensive for application to very large areas and tabulation of crop acreage information would add significantly to these costs. 
Crop maps for Chase, Dundy, and Perkins Counties in Nebraska were compiled by the Institute of Agriculture and Natural Resources at the University of Nebraska. Cropland was mapped by visually interpreting a combination of 1:80,000 scale color infrared aerial photographs and Landsat images. The aerial photographs were obtained early in the growing season and then supplemented with Landsat images obtained later in the season. The area mapped was about 2,700 square miles. Cropland maps were compiled by individual counties at a scale of approximately 1:80,000.

Cropland maps produced using this approach were generally accurate both in boundary delineation and crop-type interpretation. A comparison was made between these maps and cropland maps interpreted from the 1:24,000 color infrared photographs discussed previously. Cropland maps compiled for the same areas using these two methods were virtually the same, both in boundary delineations and crop-type interpretations. However, this approach was very time consuming and moderately expensive ( $\$ 3.00$ per square mile), which would make it unsuitable for large area applications; and acreage tabulations would add significantly to these costs.

\section{Landsat Data}

Landsat data acquired July 27, 1978, were used for both visual and digital interpretation of irrigated cropland for Phillips and Yuma Counties in Colorado. A map of irrigated cropland for the same area and season was compiled from high-resolution security classified source materials. The security classified source materials map was used as a basis for evaluation of maps generated from the visual and digital interpretation of Landsat data. Security classified source materials were not considered a viable source for general mapping of the High Plains region because of the restricted availability of these materials. Interpretations of Landsat data and security classified source materials were conducted by personnel from the U.S. Geological Survey. A brief summary of the results of the Landsat mapping effort is presented here; a detailed discussion of analysis procedures used and the results obtained are reported by Thelin, Johnson, and Johnson (1980).

Visual interpretation of Landsat data in Phillips and Yuma Counties in Colorado was made using a color composite image. All fields with a significant infrared response were classified as irrigated cropland.

Landsat digital data were analyzed using computer compatible digital tapes of the same scene used in the visual interpretation. Irrigated cropland was readily identified in this analysis by the small digital values in the visible red (chlorophyll absorption) band and the large digital values in the infrared band. Non-irrigated cropland and rangeland were distinguished by other characteristic responses in the visible and infrared bands. Four major land-cover classes were identified: water, herbaceous rangeland, dryland cropland, and irrigated cropland. 
Landsat-derived irrigated-cropland maps were generally accurate in delineation of field boundaries within the constraints of the small scale of the imagery. The resolution of Landsat data is about 1.1 acres. A comparison of the acreages compiled from the Landsat visual and digital interpretations, with the acreage obtained from the security classified source materials map, is presented in table 5. The combined areal estimates of irrigated cropland for Phillips and Yuma Counties, Colorado, obtained from the Landsat interpretations were within 2 percent of the irrigated cropland area mapped using security classified source materials. Available ASCS records for the 1978 irrigation season were used to evaluate specific differences between the Landsat maps and the security classified source materials map. Most of the differences between the irrigated cropland map obtained by interpretation of Landsat and the irrigated cropland map compiled using security classified source materials resulted from misclassification of non-irrigated feed grain crops and some irrigated small grain crops on the Landsat image. At the time of acquisition of the Landsat scene (July 27, 1978), small grain crops had been harvested, and therefore, would not have the typical response in the infrared band associated with irrigated cropland. The map compiled from the security classified source materials correctly identified all of the fields that were checked against ASCS records.

The cost of mapping irrigated cropland and tabulating acreage for the Landsat visual and digital interpretation is estimated to be between $\$ 1.00$ and $\$ 1.50$ per square mile. These cost figures include estimates of the cost of materials, manpower, and computer time required to produce map products and tabular data of irrigated cropland on a regional scale. No attempt was made during the preliminary evaluation of Landsat mapping to provide croptype information, but some reliable crop-type information should be available from Landsat analysis especially when more than one scene covering the same area is used.

Landsat analysis alone cannot be expected to provide the detail obtained from the other mapping methods tested because of the small scale of the imagery. However, considering costs, availability of data, and time required for analysis of the methods evaluated, Landsat data appears to be the most suitable alternative for mapping irrigated cropland for an area as large as the High Plains.

\section{SUMMARY AND CONCLUSIONS}

The primary emphasis of the High Plains Regional Aquifer-System Analysis water-use program during 1979 was to develop and test methods and instrumentation to determine ground-water use for irrigated agriculture in the High Plains region. These objectives were accomplished; methods and instrumentation have been defined for determining irrigation pumpage information for the entire High Plains region during the 1980 irrigation season.

The 1979 sampling effort provided thorough testing for the VTT units and the three portable flow-measuring devices. The VTT units functioned very 
Table 5.--Irrigated cropland estimates for Phillips

and Yuma Counties, Colorado, 1978

Irrigated cropland in acres

Data source

Phillips County

Yuma County

Total

Security classified

source materials------

66,039

243,519

309,558

Landsat visual

interpretation--------

69,155

238,849

305,004

Landsat digital

interpretation---------

65,035

244,430

309,465 
well for recording time of operation of irrigation wells, with an overall failure rate of about 10 percent. Based on the positive results obtained with these prototype VTT units, a production model of this device has been selected as the primary method for determining time of operation of wells to be sampled during the 1980 irrigation season. All three portable flowmeters evaluated during the 1979 irrigation season were subjected to extensive field testing and varying degrees of laboratory testing. Only the transient-time flowmeter provided reliable measurements of discharge over the variety of irrigation systems in use in the High Plains. Consequently, the transient-time flowmeter has been selected as the principal flow-measuring device for use in pumpage sampling during the 1980 irrigation season.

Pumpage rates for each of the wells on which reliable time of operation and discharge values were obtained were combined with the irrigated crop acreage information to determine the annual depth of application of water. Statistical analyses of these application rates indicated that the mean application rate for all crops and systems in the two test areas could be estimated from sample data within 1.4 inches in the northern test area and within 1.0 inch in the southern test area at the 95 percent confidence level. Statistical analyses of pumpage data separated by irrigation system type indicated that there is a significant difference, at the 95 percent confidence level, in application rates between sprinklèr irrigation systems and flood irrigation systems with sprinkler irrigation systems applying the lesser amount of water. Tests for significant differences in application rates for different crop types indicated significant differences occurred between corn and alfalfa for sprinkler irrigation systems, but not for flood irrigation systems. Test for climatic effects on application rates in the four quadrants of the northern test area indicated no significant differences were present.

Five methods for mapping irrigated cropland in the High Plains region were investigated. The four methods using reported data, physical inventory, and two scales of aerial photographs were found to be prohibitively expensive for mapping irrigated cropland over the entire High Plains region. A method that used only Landsat data proved to be readily adaptable to large area mapping of irrigated cropland. The small scale, low cost of analysis, and repetitive coverage of the Landsat data were all factors that contributed to the selection of Landsat as the primary data source for providing irrigated cropland maps of the High Plains region during 1980. Digital analysis will be used whenever possible because of the ease with which the interpreted data can be compiled and stored in a computerized data base.

The data collected and analyzed during 1979 and presented in this report have been used to define a pumpage sampling and cropland mapping approach to be applied to the entire High Plains region during the 1980 irrigation season. This approach is presented in the section entitled "1980 Water-Use Plan." 


\section{WATER-USE PLAN}

Ten pumpage monitoring areas consisting of all or parts of 14 counties located in various parts of the High Plains region have been selected for the 1980 irrigation season (fig. 7). Approximately 50 irrigation sites will be randomly selected within each of the areas. No stratification of crops or irrigation systems has been designed in the selection procedure. The distribution of the 1980 monitoring areas was designed to provide a representative cross section of irrigation systems, crop types, and physical factors such as geology, hydrology, soils, and climate that occur within the High Plains region.

The primary instrument to be used to measure discharge of wells will be the transient-time flowmeter. Additionally, one well in some of the monitoring areas will be equipped with a calibrated inline flowmeter for comparison with the transient-time meter. The discharge will be measured at each well at least one time during the irrigation season and at least 20 percent of the we11s will be measured more than once. The purpose of the multiple measurements is to define any seasonal trends in discharge. Time of operation at each well site will be monitored using a production model of the VTT units. Two VTT units will be installed on each well to insure the reliability of the time measurement and to evaluate the variability among the VTT units under field condiitions. Other available sources of time of operation also will be monitored. Crop type and acreage irrigated for each pumpage monitoring site will be obtained by measurement, reported information, or personal contact with individual irrigators.

Irrigated cropland maps for the entire High Plains region are currently being prepared using 1978 Landsat imagery. This extension of the 1979 effort is being conducted to define the coverage required, the ability of the Landsat system to provide crop-type information, and the suitability of Landsat images for mapping irrigated cropland in higher precipitation regions.

Thirty-eight Landsat scenes are required to provide complete coverage of the entire region (fig. 8). Two or possibly three scenes at different times of the irrigation season will be required in the southern part of the High Plains to cover the variety of crop growth cycles that result from a longer growing season in this region. Only limited crop-type information probably will be provided by Landsat analysis alone. However, regularly reported agricultural statistics should provide crop-type information at a resolution suitable for use with a regional ground-water model.

Some problems may be encountered in using Landsat data to map irrigated cropland in some of the areas in the eastern High Plains that receive greater precipitation. It may be difficult to discriminate between dryland and irrigated crops in these areas using Landsat data alone. The 1978 Landsat data will be used to evaluate the seriousness of the problem and to define areas where alternative approaches and data sources may have to be used as a supplement or in lieu of Landsat data. 


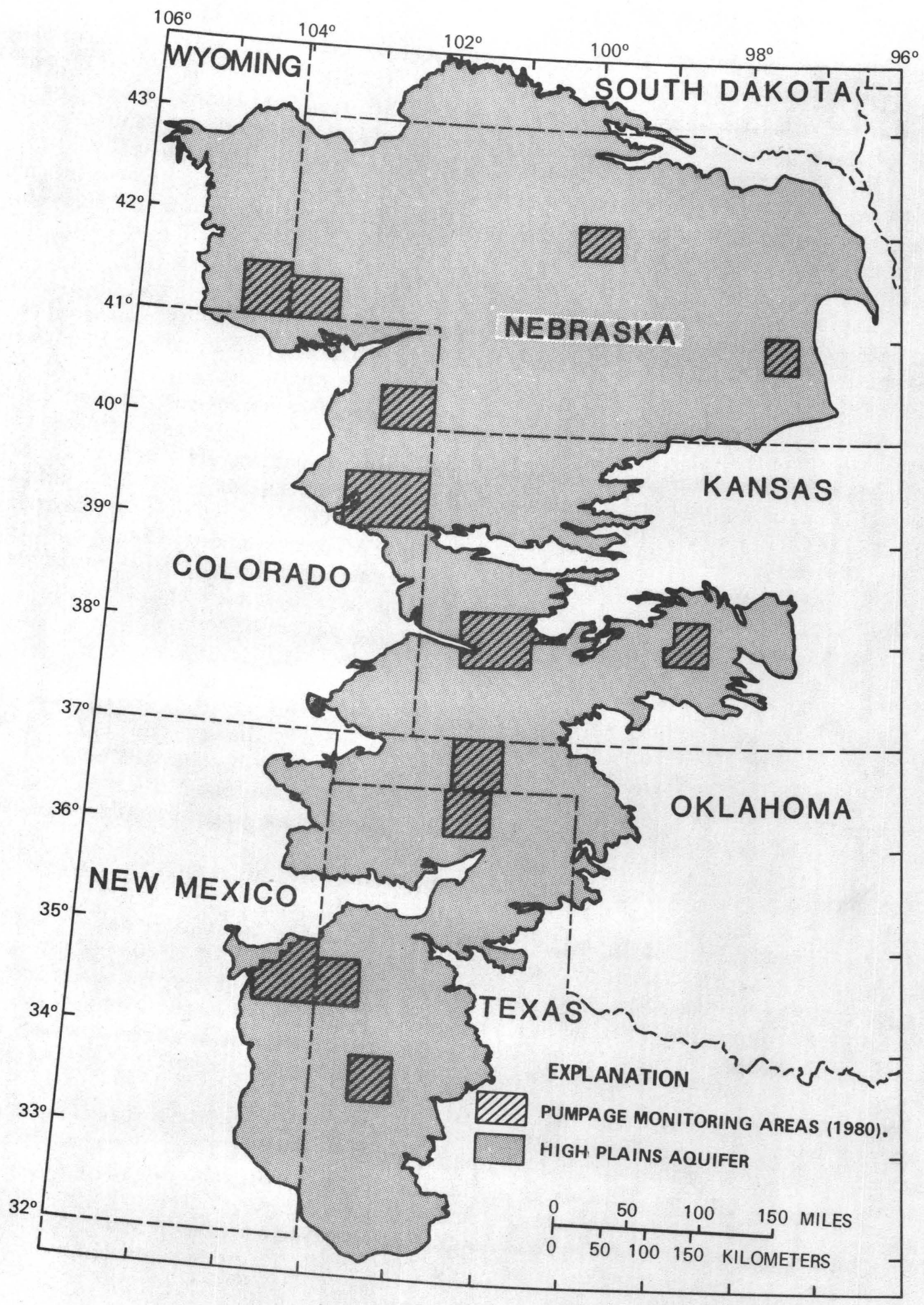

Figure 7.-- Location of 1980 pumpage monitoring areas. 


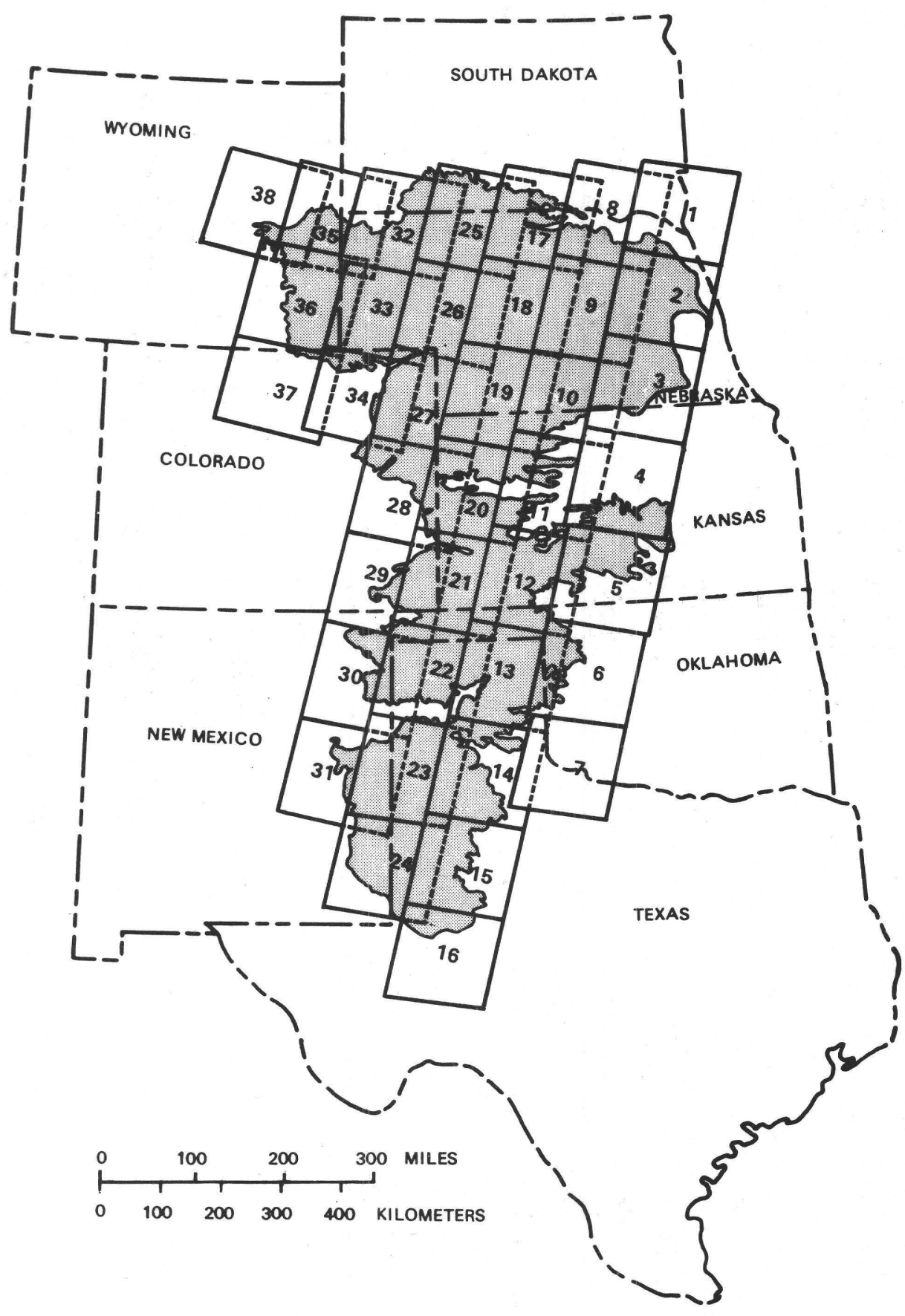

Figure 8.--Number and location of Landsat scenes required to provide coverage of the High Plains aquifer. 
Based on the evaluation of the 1978 Landsat data and investigations of other data sources for mapping irrigated cropland, a composite approach will be defined for mapping irrigated cropland for the entire High Plains region during the 1980 irrigation season.

Irrigated cropland information obtained from Landsat analysis will be digitally output in grid-cell format for use with the regional data base being developed for the High Plains Regional Aquifer-System Analysis (Weeks, 1978). This irrigated acreage data will be combined with information obtained in ground sampling from the pumpage monitoring areas to provide pumpage estimates by grid-cell. The pumpage estimates will be combined in a groundwater flow model with data on aquifer properties (specific yield and hydraulic conductivity), aquifer geometry (top of aquifer, base of aquifer, and lateral boundaries), and other stresses (natural recharge, recharge from dryland cropland, recharge from irrigated cropland, and cross-boundary flow). The model will simulate historical changes in water levels and will be calibrated to obtain the best correspondence between simulated water-level changes and measured water-level changes. The model then will be used to calculate the future changes in water levels caused by different management alternatives and thus will aid in planning the future course of irrigation on the High Plains. 


\section{REFERENCES CITED}

Grubb, H. W., 1978, High Plains study: Fifth Annual Groundwater Management Districts Association Conference, Amaril1o, Texas, December 6-8, 1978, p. 3-12.

Gutentag, E. D., and Weeks, J. B., 1980, Water table in the High Plains aquifer in 1978 in parts of Colorado, Kansas, Nebraska, New Mexico, Oklahoma, South Dakota, Texas, and Wyoming: U.S. Geological Survey Hydrologic Investigations Atlas, HA-642.

Lohman, S. W., 1953, High Plains of west-central United States, general aspects, in Subsurface facilities of water management and patterns of supply-type area studies, chap. 4, (in The Physical and Economic Foundation of Natural Resources, v. 4): U.S. 83rd Congress, House Committee on Interior and Insular Affairs, p. 70-78.

Luckey, R. R., Heimes, F. J., and Gaggiani, N. G., 1980, Calibration and testing of selected portable flowmeters for use on large irrigation systems: U.S. Geological Survey Water-Resources Investigations 80-72, $21 \mathrm{p}$.

Thelin, G. P., Johnson, T. L., and Johnson, R. A., 1980, Mapping irrigated cropland on the High Plains using Landsat: Fifth Annual Pecora Symposium, Sioux Falls, South Dakota, June 1979 [in press].

Weeks, J. B., 1978, Plan of study for the High Plains regional aquifer systems analysis in parts of Colorado, Kansas, Nebraska, New Mexico, Oklahoma, South Dakota, Texas, and Wyoming: U.S. Geological Survey Water-Resources Investigations $78-70,28 \mathrm{p}$. 


\section{Pumpage Data for Irrigation Sites}

The measurements contained in the following tables were made during the 1979 irrigation season. These data were collected in support of an effort to determine viable techniques and instrumentation that could be used for irrigation pumpage sampling in the High Plains region during 1980 .

The data shown in these tables are experimental data and as such any particular measurement may be subject to large errors. The 1979 pumpage sampling program was designed to test techniques and instrumentation and as the data in this supplement clearly indicate, some of the techniques and instruments tested were not appropriate.

\section{Explanation of Terms for Tables 6-15}

TABLE 6-15a--CROP AND WELL INFORMATION

Site Location

Local numbering system used for tables 6-13; see figure 9. Latitude and longitude used for tables 14-15.

\section{Station Identifier}

The "station identifier" is a 15-digit number generated from the original latitude and longitude of the site in degrees, minutes, and seconds with a 2-digit sequence code at the end to locate multiple sites within a 1-second area. The station identifier is unique for each site and is not changed once it has been entered into the U.S. Geological Survey's computer files.

\section{Irrigated Crop Information}

Type --Type of crop(s) irrigated at the site

System--Type of irrigation system used:

Flood = Ditch or gated pipe

Pivot = Center pivot

Sprinkler $=$ Sprinklers other than the center pivot

Acres --Acreage of crop(s) irrigated at the site

Source--Source of acreage information:

Meas. = Measured acreage

Rept. = Reported acreage

Est. = Estimated 


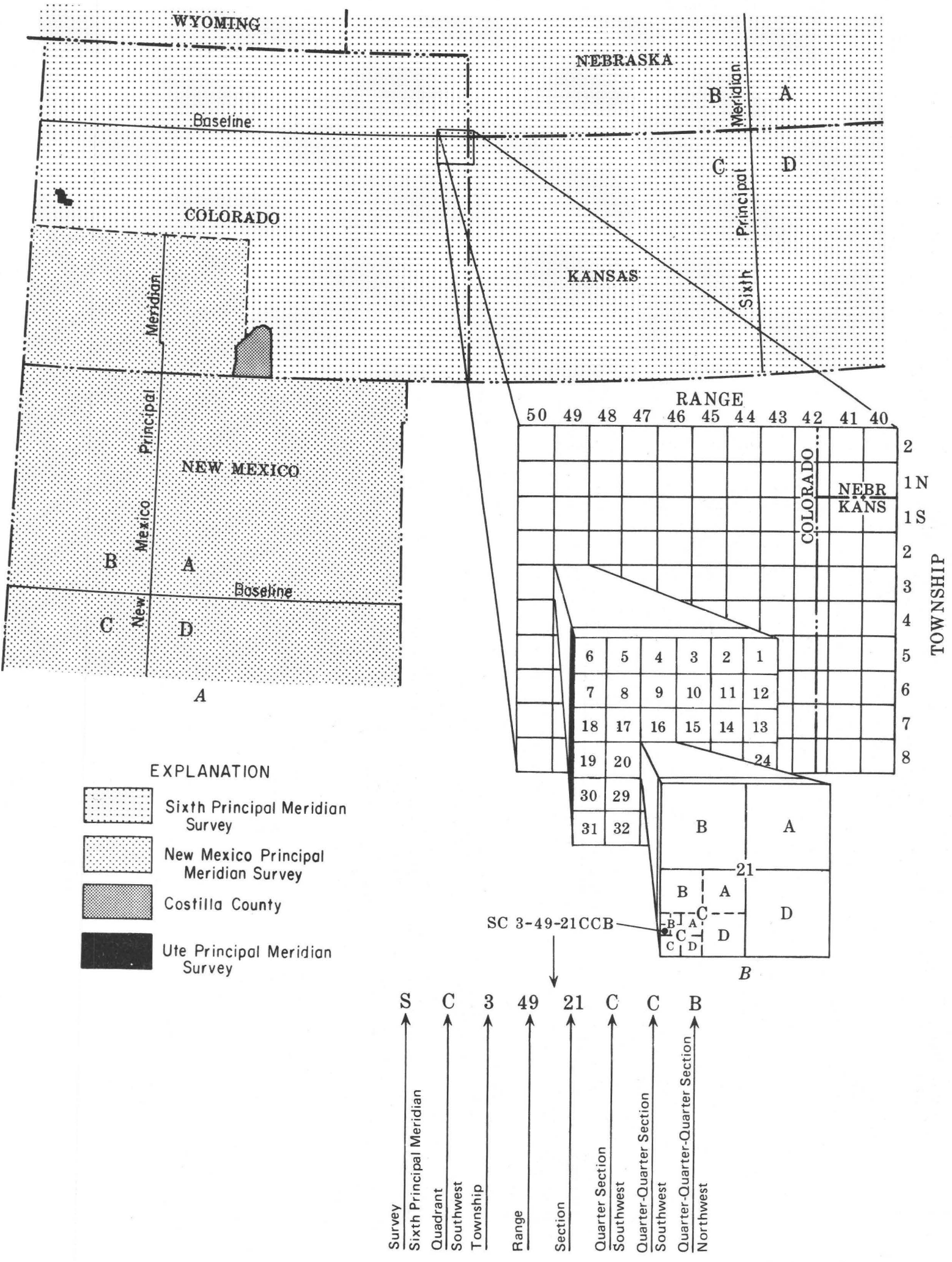

Figure 9.-- Site-location numbering system for tables 6-13. 
Depth --Depth of we11, in feet below land surface

Water level --Depth to water, in feet below land surface

Diameter --Diameter of well casing, in inches

Pump H.P. --Rated pump motor horsepower from motor plate Energy source--Pump power source:

Elec. = Electrical pump

N. gas = Natural gas engine

Energy rating--

$$
\text { Diese1 }=\text { Diese1 engine }
$$
Electric meter
= Instantaneous kilowatt
Natural gas meter = Instantaneous natural gas demand, in cubic feet per minute

TABLE 6-15b--DISCHARGE INFORMATION

\section{Site Location}

Local numbering system used for tables 6-13; see figure 9.

Latitude and longitude used for tables 14-15.

Field Person --Initials of field person(s) who collected the data

Date --Date when measurement was made

Discharge --Discharge, in gallons per minute

Method --Method used to obtain discharge:
T.T. = Transient time meter
Dop. = Doppler meter
In1. = Inline flowmeter
Buck. = Bucket and stopwatch
Prop. = Propeller meter
Rept. = Reported
Traj. = Trajectory
Hoff = Hoff meter
Other $=$ See remarks

TABLE 6-15c--TIME OF OPERATION

\section{Site Location}

Local numbering system used for tables 6-13; see figure 9. Latitude and longitude used for tables 14-15. 
Field Person --Initials of field person(s) who collected the data

Begin date --Beginning date of time measurement

End date --Ending date of time measurement

Sentry time ${ }^{1} \quad$ - -Time recorded by Vibration Time Totalizer (Sentry), in hours

Energy meter --Type of meter:

Elec. = Electric meter

N. gas = Natural gas meter

Energy meter time ${ }^{1}$--Time computed from energy meter, in hours Other time ${ }^{l} \quad$--Time from other sources, explained in remarks

${ }^{1}$ Total time is 1isted only if the total time of operation was recorded for the irrigation season. 
Table 6a.--Pumpage data for ixrigation sites in Kit Carson County, Colorado

\begin{tabular}{|c|c|c|c|c|c|c|c|c|c|c|c|c|}
\hline \multirow{3}{*}{$\begin{array}{c}\text { Site } \\
\text { location }\end{array}$} & \multirow{3}{*}{$\begin{array}{l}\text { Station } \\
\text { identifier }\end{array}$} & \multicolumn{4}{|c|}{ Crop } & \multicolumn{6}{|c|}{ Well information } & \multirow{3}{*}{ Remarks } \\
\hline & & \multirow{2}{*}{ Type } & \multirow{2}{*}{ System } & \multirow{2}{*}{ Acres } & \multirow{2}{*}{ Source } & \multirow{2}{*}{$\begin{array}{l}\text { Depth } \\
(\mathrm{ft})\end{array}$} & \multirow{2}{*}{$\begin{array}{l}\text { Water } \\
\text { level } \\
\text { (ft) }\end{array}$} & \multirow{2}{*}{$\begin{array}{l}\text { Diam- } \\
\text { eter } \\
\text { (in) }\end{array}$} & \multirow{2}{*}{$\begin{array}{l}\text { Pump } \\
\text { hp. }\end{array}$} & \multicolumn{2}{|c|}{ Power } & \\
\hline & & & & & & & & & & Source & Rating & \\
\hline$S C-06-42-11-C A D$ & 393201102034200 & Corn & Flood & 160 & Meas. & 320 & -- & 16 & 100 & Elec. & 70 & \\
\hline SC-06-43-09-DCA & 393205102123800 & Corn & Pivot & 120 & Meas. & 280 & 190 & 16 & -- & N. gas & - & Water-level measurement on $5 / 16 / 79$. \\
\hline SC-06-43-11-BAC1 & 393230102103201 & $\begin{array}{l}\text { Corn } \\
\text { Alfalfa }\end{array}$ & Flood & $\begin{array}{l}60 \\
10\end{array}$ & $\begin{array}{l}\text { Rept. } \\
\text { Rept. }\end{array}$ & 140 & 46 & -- & 50 & Elec. & 40 & Water-level measurement on $5 / 7 / 79$ \\
\hline SC-06-45-31-AAC1 & - 392413102280401 & $\begin{array}{l}\text { Corn } \\
\text { Alfalfa }\end{array}$ & $\begin{array}{l}\text { Pivot } \\
\text { Pivot }\end{array}$ & $\begin{array}{l}120 \\
120\end{array}$ & $\begin{array}{l}\text { Rept. } \\
\text { Rept. }\end{array}$ & -- & -- & -- & 100 & Elec. & -- & $\begin{array}{l}\text { These fields also were irrigated } \\
\text { from other wells during } 1979 . \\
\text { Site not included in statistical } \\
\text { summary. }\end{array}$ \\
\hline $\mathrm{SC}-06-46-08-\mathrm{DBD} 1$ & 393217102334101 & Corn & Pivot & 120 & Rept. & 224 & -- & 18 & 100 & Elec. & $-\cdots$ & \\
\hline SC-06-50-29-CCB & 392930103012000 & Corn & Flood & 80 & Rept. & 167 & 141 & -- & 50 & Elec. & 36 & $\begin{array}{l}\text { Water-ieve } 1 \text { measurement on } 10 / 3 / 55 \text {. } \\
\text { Pump had } 40 \mathrm{hp.} \mathrm{motor} \mathrm{early} \mathrm{in} \\
\text { season. }\end{array}$ \\
\hline SC-07-42-17-ACC & 392642102064400 & $\begin{array}{l}\text { Alfalfa } \\
\text { Grass }\end{array}$ & Pivot & $\begin{array}{r}120 \\
50\end{array}$ & $\begin{array}{l}\text { Rept. } \\
\text { Rept. }\end{array}$ & 317 & 194 & 16 & -- & N. gas & -- & Water-leve 1 measurement, $2 / 70$ \\
\hline
\end{tabular}

SC-07-43-23-ACA 392536102102000 Corn Flood 80 Meas. $307 \quad 170 \quad 16 \quad$-- N. gas -- Water-1evel measurement, $5 / 71$ 
Table 6b.--Fumpage data for irrigation sites in Kit Carson County, Colorado

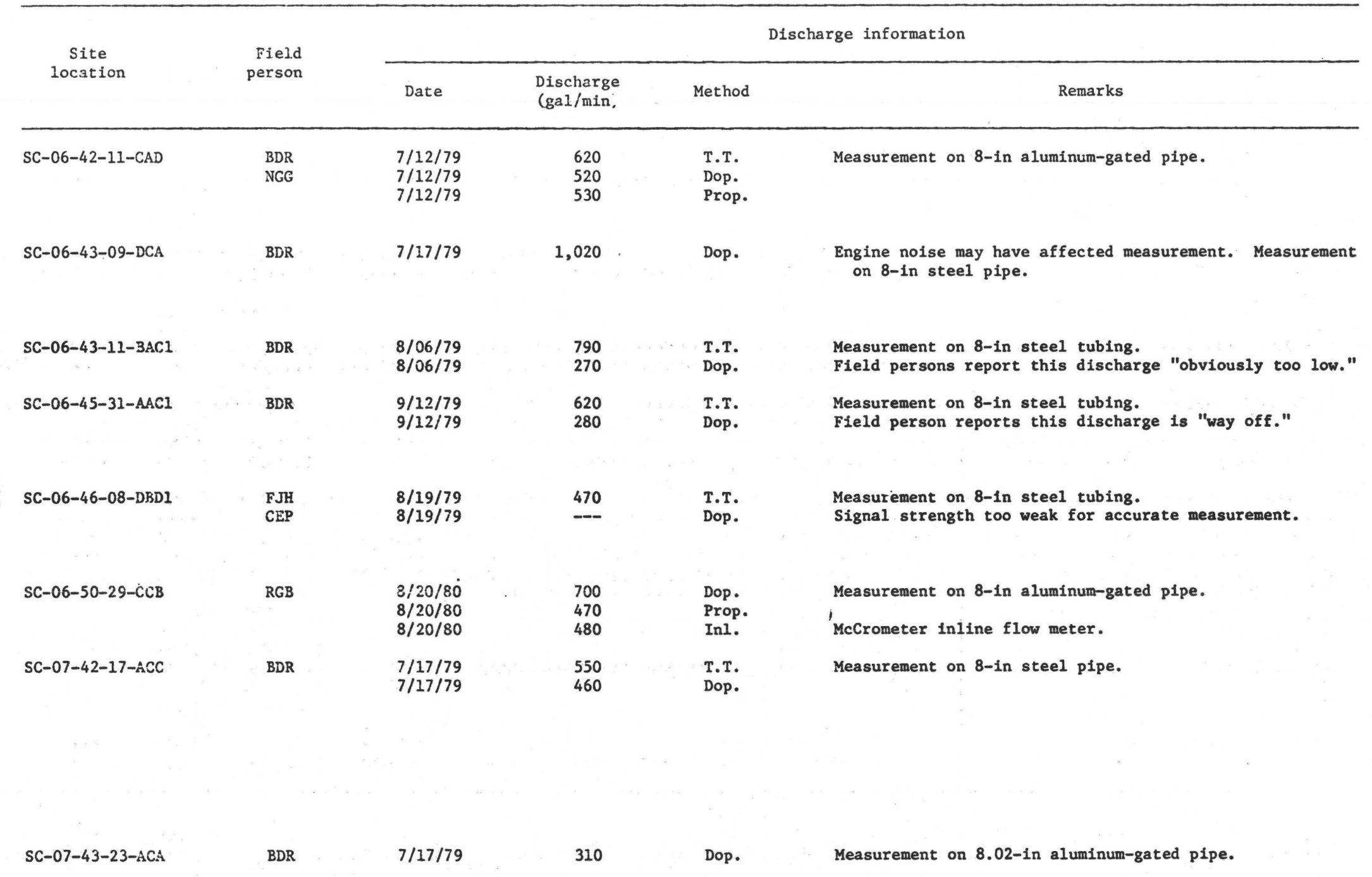


Table 6c.--Pumpage data for impigation sites in Kit Carson County, Coloradi

\begin{tabular}{|c|c|c|c|c|c|c|c|c|c|}
\hline \multirow{3}{*}{$\begin{array}{l}\text { Site } \\
\text { location }\end{array}$} & \multirow{3}{*}{$\begin{array}{l}\text { Field } \\
\text { person }\end{array}$} & \multicolumn{8}{|c|}{ Time of operation information } \\
\hline & & \multicolumn{2}{|c|}{ Date } & \multirow{2}{*}{$\begin{array}{l}\text { Sentry } \\
\text { time } \\
\text { (hours) }\end{array}$} & \multicolumn{2}{|c|}{ Energy meter } & \multicolumn{2}{|c|}{ Other } & \multirow{2}{*}{ Remarks } \\
\hline & & Begin & End & & Type & $\begin{array}{l}\text { Time } \\
\text { (hours) }\end{array}$ & Source & $\begin{array}{l}\text { Time } \\
\text { (hours) }\end{array}$ & \\
\hline \multirow[t]{2}{*}{ SC-06-42-11-CAD } & $\begin{array}{l}\text { BDR } \\
\text { NG }\end{array}$ & $\begin{array}{l}5 / 07 / 79 \\
7 / 12 / 79\end{array}$ & $\begin{array}{r}7 / 12 / 79 \\
10 / 22 / 79\end{array}$ & $\begin{array}{r}252 \\
1,120\end{array}$ & $\overline{-}$ & $\overline{--}$ & $\overline{--}$ & -- & Sentry 339. \\
\hline & & & Totai- & 1,372 & & & & & \\
\hline \multirow[t]{2}{*}{$S C-06-43-09-D C A$} & BER & $\begin{array}{l}5 / 16 / 79 \\
7 / 17 / 79\end{array}$ & $\begin{array}{r}7 / 17 / 79 \\
10 / 22 / 79\end{array}$ & $\begin{array}{l}166 \\
827\end{array}$ & $\overline{--}$ & $\overline{--}$ & $\overline{--}$ & $\overline{-\infty}$ & Sentry 296. \\
\hline & & & Total- & 993 & & & & & \\
\hline SC-06-43-11-BAC1 & BDR & $\begin{array}{l}6 / 26 / 79 \\
8 / 06 / 72\end{array}$ & $\begin{array}{r}8 / 06 / 79 \\
10 / 22 / 79\end{array}$ & $-\overline{486}$ & Elec. & 270 & $\overline{--}$ & $=$ & $\begin{array}{l}\text { Sentry lost. } \\
\text { Sentries } 496 \text { and } 497 .\end{array}$ \\
\hline SC-06-45-31-AACI & EDR & - & $9 / 12 / 79$ & -- & - & -- & Rept. & 3,480 & $\begin{array}{l}\text { Other time reported by owner. Sentry } \\
\text { lost when pump pulled. }\end{array}$ \\
\hline \multirow[t]{2}{*}{ SC-06-46-08-DBD1. } & $\begin{array}{l}\text { FJM } \\
\text { BDR }\end{array}$ & $\begin{array}{l}5 / 22 / 79 \\
8 / 19 / 79\end{array}$ & $\begin{array}{l}8 / 19 / 79 \\
9 / 20 / 79\end{array}$ & $\begin{array}{r}1,088 \\
298 \\
\end{array}$ & $\overline{--}$ & $\overline{--}$ & $\overline{--}$ & $\overline{--}$ & Sentry 315. \\
\hline & & & Total-: & 1,386 & & & & & \\
\hline SC-06-50-29-CCB & RGB & $5 / 24 / 79$ & $8 / 20 / 79$ & -- & Elec. & 743 & $\begin{array}{l}\text { Flow } \\
\text { meter }\end{array}$ & 760 & $\begin{array}{l}\text { Sentry 346. Sentry removed with old } \\
\text { pump. }\end{array}$ \\
\hline \multirow[t]{5}{*}{ SC-07-42-17-ACC } & BDR & - & $6 / 20 / 79$ & -- & -- & -- & $\begin{array}{l}\text { Farmer } \\
\text { estimate }\end{array}$ & 408 & $\begin{array}{l}\text { Farmer time estimate based on total } \\
\text { operation for season through } 6 / 19 / 79 \text {. }\end{array}$ \\
\hline & & $6 / 07 / 79$ & $6 / 20 / 79$ & 66 & N. gas & 70 & - & -- & $\begin{array}{l}\text { Timer installed } 6 / 7 / 79 \text {, af ter pump } \\
\text { was already operating. }\end{array}$ \\
\hline & & $6 / 20 / 79$ & $7 / 17 / 79$ & 213 & N. gas & 195 & -- & -- & $\begin{array}{l}\text { Sentry } 354 \text { installed } 6 / 7 / 79 \text {, replaced } \\
\text { by sentry } 481 \text { on } 6 / 20 / 79 \text {. }\end{array}$ \\
\hline & & $7 / 17 / 79$ & $10 / 22 / 79$ & 95 & -- & - & - & -- & $\begin{array}{l}\text { Farmer estimate through } 6 / 19 / 79 \text {, plus } \\
\text { sentry time from } 6 / 20 / 79 \text { to }\end{array}$ \\
\hline & & & Total- & 364 & & & & & $10 / 22 / 79=716$ \\
\hline \multirow[t]{4}{*}{ SC-07-43-23-ACA } & BDR & $5 / 07 / 79$ & $7 / 17 / 79$ & $\begin{array}{r}0 \\
403\end{array}$ & -- & $-\overline{a n}$ & -- & $\overline{--}$ & $\begin{array}{l}\text { Sentry unit detached from well } 5 / 7 / 79- \\
7 / 17 / 79 \text {. }\end{array}$ \\
\hline & & $8 / 06 / 79$. & $8 / 20 / 79$ & 403 & $\begin{array}{l}\text { N. gas } \\
\text { N. gas }\end{array}$ & $\begin{array}{l}382 \\
233\end{array}$ & - & $\ldots$ & Sentry 341 . \\
\hline & & $8 / 06 / 79$ & $10 / 22 / 79$ & 488 & -- & $\cdots$ & -- & -- & \\
\hline & & & Total- & 891 & & & & & Sentry total $7 / 17 / 79-10 / 22 / 79$. \\
\hline
\end{tabular}


Table 6a.--Pumpage data for irrigation sites in Kit Carson County, Colorado--Continued

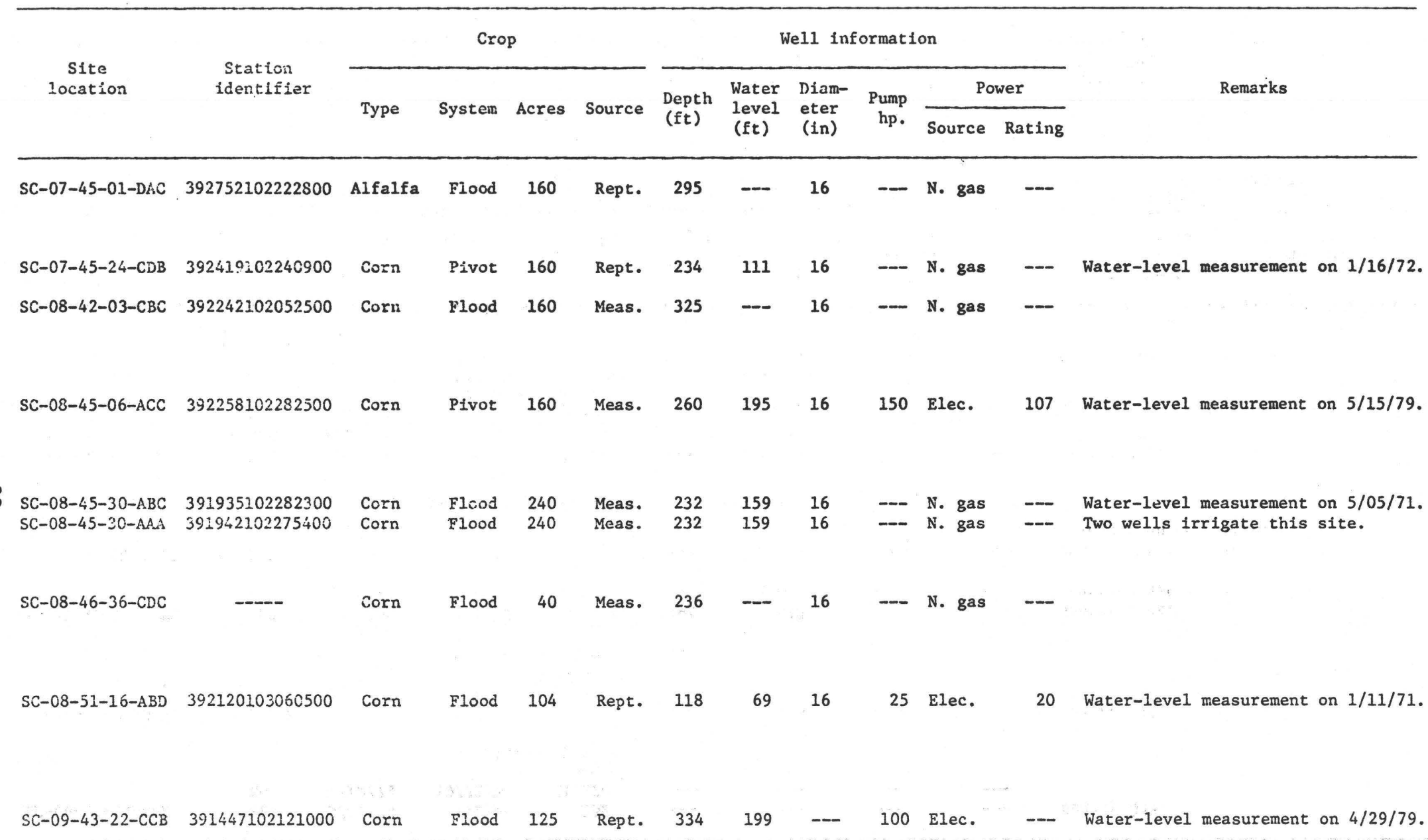


Table 6b.--Pumpage data for irrigation sites in Kit Carson County, Colorado--Continued

\begin{tabular}{|c|c|c|c|c|c|}
\hline \multirow{2}{*}{$\begin{array}{l}\text { Site } \\
\text { location }\end{array}$} & \multirow{2}{*}{$\begin{array}{l}\text { Field } \\
\text { person }\end{array}$} & \multicolumn{4}{|c|}{ Discharge information } \\
\hline & & Date & $\begin{array}{l}\text { Discharge } \\
\text { (gal/min) }\end{array}$ & Method & Remarks \\
\hline SC-07-45-01-DAC & CEP & $9 / 07 / 79$ & 760 & T.T. & Measurement on $8.02-$ in aluminum-gated pipe. \\
\hline $\mathrm{SC}-07-45-24-\mathrm{CDB}$ & CEP & $8 / 31 / 79$ & 910 & T.T. & Measurement on 8-in steel tubing. \\
\hline $\mathrm{SC}-08-42-03-\mathrm{CBC}$ & $\mathrm{BDR}$ & $\begin{array}{l}7 / 17 / 79 \\
7 / 17 / 79 \\
7 / 17 / 79\end{array}$ & $\begin{array}{l}910 \\
820 \\
760\end{array}$ & $\begin{array}{l}\text { T.T. } \\
\text { Dop. } \\
\text { Prop. }\end{array}$ & Measurement on $8.02-$ in aluminum-gated pipe. \\
\hline SC-08-45-06-ACC & $\begin{array}{l}\text { BDR } \\
\text { NGG }\end{array}$ & $\begin{array}{l}7 / 10 / 79 \\
7 / 10 / 79\end{array}$ & $\begin{array}{l}430 \\
540\end{array}$ & $\begin{array}{l}\text { T.T. } \\
\text { Dop. }\end{array}$ & $\begin{array}{l}\text { Measurement on } 8 \text {-in steel tubing. } \\
\text { Measurement on } 10-\text { in pipe. }\end{array}$ \\
\hline $\begin{array}{l}\text { SC- } 08-45-30-A B C \\
\text { SC- } 08-45-30-A A A\end{array}$ & $\begin{array}{l}\mathrm{BDR} \\
\mathrm{CH}\end{array}$ & $\begin{array}{l}6 / 19 / 79 \\
6 / 19 / 79\end{array}$ & $\begin{array}{l}980 \\
780\end{array}$ & $\begin{array}{l}\text { Dop. } \\
\text { Prop. }\end{array}$ & $\begin{array}{l}\text { Measurement on 10-in aluminum-gated pipe. } \\
\text { Discharge is for two wells combined. }\end{array}$ \\
\hline$S C-08-46-36-C D C$ & $\begin{array}{l}\mathrm{BDR} \\
\mathrm{CH}\end{array}$ & $\begin{array}{l}6 / 19 / 79 \\
6 / 19 / 79\end{array}$ & $\begin{array}{l}420 \\
230\end{array}$ & $\begin{array}{l}\text { Dop. } \\
\text { Prop. }\end{array}$ & Measurement on 6-in aluminum-gated pipe. \\
\hline$S C-08-51-16-A B D$ & BDR & $\begin{array}{l}8 / 08 / 79 \\
8 / 08 / 79\end{array}$ & $\begin{array}{l}520 \\
190\end{array}$ & $\begin{array}{l}\text { Dop. } \\
\text { Prop. }\end{array}$ & Measurement on 8-in aluminum-gated pipe. \\
\hline $\mathrm{SC}-09-43-22-\mathrm{CCB}$ & $\begin{array}{l}\text { BDR } \\
\text { NGG }\end{array}$ & $\begin{array}{l}7 / 12 / 79 \\
7 / 12 / 79 \\
8 / 07 / 79 \\
8 / 07 / 79\end{array}$ & $\begin{array}{l}530 \\
490 \\
630 \\
670\end{array}$ & $\begin{array}{l}\text { Buck. } \\
\text { Dop. } \\
\text { T.T. } \\
\text { Dop. }\end{array}$ & $\begin{array}{l}\text { Measurement ali gates. } \\
\text { Measurement on } 8 \text {-in aluminum-gated pipe. }\end{array}$ \\
\hline
\end{tabular}


Table 6c.--Pumpage data for irrigation sites in Kit Carson County, Colorado--Continued

\begin{tabular}{|c|c|c|c|c|c|c|c|c|c|}
\hline \multirow{3}{*}{$\begin{array}{l}\text { Site } \\
\text { location }\end{array}$} & \multirow{3}{*}{$\begin{array}{r}\text { Field } \\
\text { person }\end{array}$} & \multicolumn{8}{|c|}{ Time of operation information } \\
\hline & & \multicolumn{2}{|c|}{ Date } & \multirow{2}{*}{$\begin{array}{l}\text { Sentry } \\
\text { time } \\
\text { (hours) }\end{array}$} & \multicolumn{2}{|c|}{ Energy meter } & \multicolumn{2}{|c|}{ Other } & \multirow{2}{*}{ Remarks } \\
\hline & & Begin & End & & Type & $\begin{array}{c}\text { Time } \\
\text { (hours) }\end{array}$ & Source & $\begin{array}{l}\text { Time } \\
\text { (hours) }\end{array}$ & \\
\hline \multirow[t]{2}{*}{ SC-07-45-01-DAC } & CEP & $6 / 07 / 79$ & $10 / 20 / 79$ & 1,763 & N. gas & -- & -- & --- & Sentry 357. \\
\hline & & & Total-- & $-1,763$ & & & & & \\
\hline$S C-07-45-24-C D B$ & CEP & $5 / 16 / 79$ & $10 / 20 / 79$ & 1,491 & N. gas & -- & -- & -- & Sentry 292. \\
\hline \multirow[t]{2}{*}{ SC-08-42-03-CBC } & $\mathrm{BDR}$ & $\begin{array}{l}6 / 20 / 79 \\
7 / 17 / 79\end{array}$ & $\begin{array}{r}7 / 17 / 79 \\
10 / 22 / 79\end{array}$ & $\begin{array}{l}313 \\
762 \\
\end{array}$ & N. gas & 369 & -- & -- & Sentry 342. \\
\hline & & & Tota1-- & $-1,075$ & & & & & \\
\hline \multirow[t]{2}{*}{$\mathrm{SC}-08-45-06-\mathrm{ACC}$} & $\begin{array}{l}\text { BDR } \\
\text { NGG }\end{array}$ & $\begin{array}{l}5 / 15 / 79 \\
7 / 10 / 79\end{array}$ & $\begin{array}{l}7 / 10 / 79 \\
8 / 20 / 79\end{array}$ & $\begin{array}{r}348 \\
1,040 \\
\end{array}$ & Elec. & 382 & -- & -- & Sentry 284 . \\
\hline & & & Total-- & $-1,388$ & & & & & \\
\hline \multirow[t]{2}{*}{$\begin{array}{l}S C-08-45-30-A B C \\
S C-08-45-30-A A A\end{array}$} & $\begin{array}{l}\mathrm{BDR} \\
\mathrm{CH}\end{array}$ & $\begin{array}{l}6 / 06 / 79 \\
6 / 19 / 79\end{array}$ & $\begin{array}{r}6 / 19 / 79 \\
10 / 20 / 79\end{array}$ & $\begin{array}{r}306 \\
1,263 \\
\end{array}$ & N. gas & 281 & -- & -- & $\begin{array}{l}\text { Sentry } 377 \text {. } \\
\text { Both wells operate together. }\end{array}$ \\
\hline & & & Total-: & $-1,569$ & & & & & \\
\hline \multirow[t]{2}{*}{$S C-08-46-36-C D C$} & $\begin{array}{l}\mathrm{BDR} \\
\mathrm{CH}\end{array}$ & $\begin{array}{l}5 / 01 / 79 \\
6 / 19 / 79\end{array}$ & $\begin{array}{r}6 / 19 / 79 \\
10 / 20 / 79\end{array}$ & $\begin{array}{l}567 \\
940 \\
\end{array}$ & -- & -- & -- & -- & $\begin{array}{l}\text { Sentry } 367 \text {. Sentry found detached } \\
\text { from pump on } 6 / 19 / 79 \text {, reattached } \\
\text { after readout. }\end{array}$ \\
\hline & & & Total-- & $-1,507$ & & & & & \\
\hline \multirow[t]{2}{*}{$S C-08-51-16-A B D$} & $\mathrm{BDR}$ & $\begin{array}{l}5 / 24 / 79 \\
5 / 24 / 79 \\
8 / 08 / 79\end{array}$ & $\begin{array}{r}8 / 08 / 79 \\
8 / 20 / 79 \\
10 / 22 / 79\end{array}$ & $\begin{array}{c}1,006 \\
-- \\
408\end{array}$ & Elec. & $1, \overline{042}$ & $\begin{array}{l}--- \\
---\end{array}$ & -- & Sentry 345 \\
\hline & & & Tota1-- & $-1,414$ & & & & & \\
\hline$S C-09-43-22-C C B$ & BDR & $\begin{array}{l}4 / 29 / 79 \\
7 / 12 / 79 \\
8 / 07 / 79\end{array}$ & $\begin{array}{r}7 / 12 / 79 \\
8 / 07 / 79 \\
10 / 22 / 79\end{array}$ & $\begin{array}{l}881 \\
535 \\
547\end{array}$ & $\overline{--}$ & -- & --- & -- & Sentry 288 . \\
\hline
\end{tabular}


Table 6a.--Pumpage data for irrigation sites in Kit Carson County, Colorado--Continued

\begin{tabular}{|c|c|c|c|c|c|c|c|c|c|c|c|c|}
\hline \multirow{3}{*}{$\begin{array}{c}\text { Site } \\
\text { location }\end{array}$} & \multirow{3}{*}{$\begin{array}{c}\text { Station } \\
\text { ićentilier }\end{array}$} & \multicolumn{4}{|c|}{ Crop } & \multicolumn{6}{|c|}{ Well information } & \multirow{3}{*}{ Remarks } \\
\hline & & \multirow{2}{*}{ Type } & \multirow{2}{*}{ System } & \multirow{2}{*}{ Acres } & \multirow{2}{*}{ Source } & \multirow{2}{*}{$\begin{array}{l}\text { Depth } \\
\text { (ft) }\end{array}$} & \multirow{2}{*}{$\begin{array}{l}\text { Water } \\
\text { level } \\
\text { (ft) }\end{array}$} & \multirow{2}{*}{$\begin{array}{l}\text { Diam- } \\
\text { eter } \\
\text { (in) }\end{array}$} & \multirow{2}{*}{$\begin{array}{l}\text { Pump } \\
\text { hp. }\end{array}$} & \multicolumn{2}{|c|}{ Power } & \\
\hline & & & & & & & & & & Source & Rating & \\
\hline SC-09-43-29-DBC & 391332102135100 & Corn & Pivot & 110 & Rept. & 335 & 185 & 30 & 125 & Elec. & 118 & Water-level measurement, $5 / 71$ \\
\hline $\mathrm{SC}-09-44-32-\mathrm{ACA}$ & 391327102201600 & Alfalfa & Pivot & 140 & Rept. & 255 & -- & 16 & -- & N. gas & -- & \\
\hline SC-09-47-19-CDDI & 391438102415301 & Corn & Flood & 25 & Rept. & -- & -- & -- & -- & N. gas & - & \\
\hline$S C-10-44-10-B C C$ & 391147102185100 & Corn & Flood & 250 & Rept. & 297 & - & 16 & 100 & Elec. & 89 & \\
\hline SC-10-48-06-AAA & 391250102481000 & Corn & Flood & .126 & Rept. & 180 & -- & 16 & -- & N. gas & - & \\
\hline$S C-10-48-06-B C C$ & 391230102485000 & & & & & & & & & & - & Two wells irrigate this site. \\
\hline$S C-10-45-18-\mathrm{AAB}$ & 391109102280400 & Corn & Flood & 75 & Meas. & 292 & 197 & 16 & -- & N. gas & -- & Water-level measurement on $6 / 6 / 79$. \\
\hline$S C-11-47-27-A B B$ & 390400102394000 & Corn & Flood & 154 & Rept. & 227 & 145 & 16 & -- & N. gas & - & Water-level measurement on $5 / 1 / 79$. \\
\hline $\mathrm{SC}-11-49-26-\mathrm{BBBI}$ & 390405102515601 & Corn & Pivot & 130 & - & 205 & 151 & 16 & 60 & Elec. & - & Water-level measurement on $5 / 1 / 79$. \\
\hline
\end{tabular}


Table 6b.--Pumpage data for irrigation sites in Kit Carson County, Colorado--Continued

\begin{tabular}{|c|c|c|c|c|c|}
\hline \multirow{2}{*}{$\begin{array}{l}\text { Site } \\
\text { location }\end{array}$} & \multirow{2}{*}{$\begin{array}{l}\text { Field } \\
\text { person }\end{array}$} & \multicolumn{4}{|c|}{ Discharge information } \\
\hline & & Date & $\begin{array}{l}\text { Discharge } \\
\text { (ga!/min) }\end{array}$ & Method & Remarks \\
\hline SC-09-43-29-DBC & $\begin{array}{l}\mathrm{BDR} \\
\mathrm{CH}\end{array}$ & $\begin{array}{l}6 / 18 / 79 \\
6 / 18 / 79 \\
6 / 18 / 79\end{array}$ & $\begin{array}{l}950 \\
670 \\
910\end{array}$ & $\begin{array}{l}\text { Dop. } \\
\text { T.T. } \\
\text { Prop. }\end{array}$ & Used aluminum heads on steel pipe. \\
\hline $\mathrm{SC}-09-44-32-\mathrm{ACA}$ & $\begin{array}{l}\text { BDR } \\
\text { NGG }\end{array}$ & $\begin{array}{l}6 / 27 / 79 \\
6 / 27 / 79\end{array}$ & $\begin{array}{l}480 \\
190\end{array}$ & $\begin{array}{l}\text { T.T. } \\
\text { Dop. }\end{array}$ & Measurement on 8.625 -in steel pipe. \\
\hline SC-09-47-19-CDD1 & BDR & $8 / 08 / 79$ & 300 & Dop. & Measurement on 6-in aluminum-gated pipe. \\
\hline SC-10-44-10-BCC & BDR & $\begin{array}{l}6 / 27 / 79 \\
6 / 27 / 79\end{array}$ & $\begin{array}{l}710 \\
670\end{array}$ & $\begin{array}{l}\text { Dop. } \\
\text { Prop. }\end{array}$ & Measurement on 8-in aluminum-gated pipe. \\
\hline $\begin{array}{l}\text { SC-10-48-06-AAA } \\
\text { SC-10-48-06-BCC }\end{array}$ & $\begin{array}{l}\mathrm{BDR} \\
\mathrm{CE}\end{array}$ & $\begin{array}{l}6 / 22 / 79 \\
6 / 22 / 79\end{array}$ & $\begin{array}{l}510 \\
760\end{array}$ & $\begin{array}{l}\text { Dop. } \\
\text { Prop. }\end{array}$ & $\begin{array}{l}\text { Measurement on 8-in aluminum-gated pipe. } \\
\text { Discharge is for two wells combined. }\end{array}$ \\
\hline SC-10-45-18-AAB & $\begin{array}{l}\text { BDR } \\
\mathrm{CH}\end{array}$ & $\begin{array}{l}6 / 18 / 79 \\
6 / 18 / 79\end{array}$ & $\begin{array}{l}610 \\
520\end{array}$ & $\begin{array}{l}\text { Dop. } \\
\text { Prop. }\end{array}$ & Measurement on 8-in aluminum-gated pipe. \\
\hline$S C-11-47-27-A B B$ & BDR & $\begin{array}{l}7 / 18 / 79 \\
7 / 18 / 79\end{array}$ & $\begin{array}{l}820 \\
700\end{array}$ & $\begin{array}{l}\text { Dop. } \\
\text { Prop. }\end{array}$ & Measurement on 8-in aluminum-gated pipe. \\
\hline SC-11-49-26-BBB1 & BDR & -- & 700 & Rept. & $\begin{array}{l}\text { Two wells operate one pivot; owner estimates combined } \\
\text { discharge of } 700 \mathrm{gal} / \mathrm{min} \text {. }\end{array}$ \\
\hline
\end{tabular}


Table 6c.--Pumpage data for irrigation sites in Kit Carson County, Colorado--Continued

\begin{tabular}{|c|c|c|c|c|c|c|c|c|c|}
\hline \multirow{3}{*}{$\begin{array}{l}\text { Site } \\
\text { location }\end{array}$} & \multirow{3}{*}{$\begin{array}{r}\text { Field } \\
\text { person }\end{array}$} & & & \multirow{3}{*}{$\begin{array}{l}\text { Sentry } \\
\text { time } \\
\text { (hours) }\end{array}$} & \multicolumn{5}{|c|}{ Time of operation information } \\
\hline & & \multicolumn{2}{|c|}{ Date } & & \multicolumn{2}{|c|}{ Energy meter } & \multicolumn{2}{|c|}{ Other } & \multirow{2}{*}{ Remarks } \\
\hline & & Begin & End & & Type & $\begin{array}{c}\text { Time } \\
\text { (hours) }\end{array}$ & Source & $\begin{array}{l}\text { Time } \\
\text { (hours) }\end{array}$ & \\
\hline \multirow[t]{2}{*}{$S C-09-43-29-D B C$} & $\begin{array}{l}\mathrm{BDR} \\
\mathrm{CH}\end{array}$ & $\begin{array}{l}4 / 29 / 79 \\
6 / 28 / 79\end{array}$ & $\begin{array}{r}6 / 18 / 79 \\
10 / 22 / 79\end{array}$ & $\begin{array}{l}234 \\
921\end{array}$ & - & - & -- & -- & \multirow[t]{2}{*}{ Sentry 289} \\
\hline & & & Tota1-- & $-1,155$ & & & & & \\
\hline \multirow[t]{2}{*}{$\mathrm{SC}-09-44-32-\mathrm{ACA}$} & $\mathrm{BDR}$ & $\begin{array}{l}6 / 07 / 79 \\
6 / 27 / 79\end{array}$ & $\begin{array}{r}6 / 27 / 79 \\
10 / 22 / 79\end{array}$ & $\begin{array}{r}78 \\
1,338 \\
\end{array}$ & - & $\cdots$ & $\begin{array}{l}\text { Owner } \\
\text { estimate }\end{array}$ & 132 & \multirow[t]{2}{*}{$\begin{array}{l}\text { Sentry } 353 \text { replaced with Sentry } 483 \text { on } \\
6 / 27 / 79\end{array}$} \\
\hline & & & Total-- & $-1,416$ & & & & & \\
\hline \multirow[t]{2}{*}{$S C-09-47-19-C D D 1$} & BDR & $\begin{array}{l}5 / 01 / 79 \\
6 / 21 / 79 \\
8 / 08 / 79\end{array}$ & $\begin{array}{l}6 / 21 / 79 \\
8 / 08 / 79 \\
9 / 20 / 79\end{array}$ & $\begin{array}{r}0 \\
492 \\
373\end{array}$ & $\ldots$ & $\overline{-\infty}$ & $\overline{-\infty}$ & $\cdots$ & \multirow[t]{2}{*}{$\begin{array}{l}\text { Sentry } 333 \text { replaced with Sentry } 482 \text { on } \\
6 / 21 / 79 \text {. Well was running prior to } \\
6 / 21 / 79 \text {. }\end{array}$} \\
\hline & & & Tota1-- & 865 & & & & & \\
\hline \multirow[t]{2}{*}{$S C-10-44-10-B C C$} & BDR & $\begin{array}{l}4 / 29 / 79 \\
6 / 27 / 79\end{array}$ & $\begin{array}{l}6 / 27 / 79 \\
9 / 20 / 79\end{array}$ & $\begin{array}{r}242 \\
2,044 \\
\end{array}$ & Elec. & 125 & $\overline{--}$ & 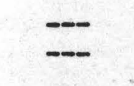 & \multirow[t]{2}{*}{ Sentry 312.} \\
\hline & & & Total-- & 2,286 & & & & & \\
\hline \multirow[t]{2}{*}{$\begin{array}{l}\text { SC-10-48-06-AAA } \\
\text { SC-10-48-06-BCC }\end{array}$} & $\begin{array}{l}\mathrm{BDR} \\
\mathrm{CH}\end{array}$ & $\begin{array}{l}5 / 01 / 79 \\
6 / 22 / 79\end{array}$ & $\begin{array}{l}6 / 22 / 79 \\
9 / 20 / 79\end{array}$ & $\begin{array}{r}598 \\
2,029 \\
\end{array}$ & - & $\overline{--}$ & $-\infty$ & $\ldots$ & \multirow[t]{2}{*}{$\begin{array}{l}\text { Sentry } 335 . \\
\text { Both wells operate together. }\end{array}$} \\
\hline & & & Total-- & $-2,627$ & & & & & \\
\hline \multirow[t]{2}{*}{$S C-10-45-18-A A B$} & $\begin{array}{l}\mathrm{BDR} \\
\mathrm{CH}\end{array}$ & $\begin{array}{l}6 / 06 / 79 \\
6 / 18 / 79\end{array}$ & $\begin{array}{l}6 / 18 / 79 \\
9 / 20 / 79\end{array}$ & $\begin{array}{r}87 \\
1,234 \\
\end{array}$ & N. gas & 65 & $\cdots$ & $\ldots$ & \multirow[t]{2}{*}{ Sentry 369.} \\
\hline & & & Total- & $-1,321$ & & & & & \\
\hline \multirow[t]{2}{*}{$S C-11-47-27-A B B$} & $\mathrm{BDR}$ & $\begin{array}{l}5 / 01 / 79 \\
7 / 18 / 79\end{array}$ & $\begin{array}{l}7 / 18 / 79 \\
9 / 20 / 79\end{array}$ & $\begin{array}{r}420 \\
1,393 \\
\end{array}$ & $\overline{-}$ & $\overline{-}$ & - & $\overline{-}$ & \multirow[t]{2}{*}{ Sentry 302} \\
\hline & & & Tota1-- & $-1,813$ & & & & & \\
\hline \multirow[t]{2}{*}{$S C-11-49-26-B B B 1$} & $\mathrm{BDR}$ & $5 / 01 / 79$ & $9 / 20 / 79$ & 514 & - & - & -- & $\cdots$ & \multirow[t]{2}{*}{ Sentry 297.} \\
\hline & & & Total-- & 514 & & & & & \\
\hline
\end{tabular}


Table 7a.--Pumpage data for irrigation sites in Phillips County, Golorado

\begin{tabular}{|c|c|c|c|c|c|c|c|c|c|c|c|c|}
\hline \multirow{2}{*}{$\begin{array}{c}\text { Site } \\
\text { location }\end{array}$} & \multirow{2}{*}{$\begin{array}{l}\text { Station } \\
\text { identifier }\end{array}$} & \multicolumn{4}{|c|}{ Irrigated crop information } & \multicolumn{6}{|c|}{ Well information } & \multirow[b]{2}{*}{ Remarks } \\
\hline & & Type & System & Acres & Source & $\begin{array}{r}\text { Depth } \\
(f t)\end{array}$ & $\begin{array}{l}\text { Water } \\
\text { level } \\
(\mathrm{ft})\end{array}$ & $\begin{array}{l}\text { Diam- } \\
\text { meter } \\
\text { (in) }\end{array}$ & $\begin{array}{c}\text { Pump } \\
\text { hp. }\end{array}$ & $\begin{array}{l}\text { Energy } \\
\text { source }\end{array}$ & $\begin{array}{l}\text { Energy } \\
\text { rating }\end{array}$ & \\
\hline$S B-06-42-07-B A B$ & 403050102052000 & Alfalfa & Flood & 150 & Rept. & 260 & 64 & 18 & 40 & Elec. & -- & Water-level measurement on $1 / 13 / 79$. \\
\hline $\mathrm{SB}-06-43-32-\mathrm{CBA}$ & 402640102102501 & Corn & Pivot & -- & -- & 354 & 92 & 16 & 75 & Elec. & -- & Water-level measurement on $3 / 8 / 79$ \\
\hline SB-06-45-11-DBA1 & 403018102204201 & Corn & Pivot & 125 & Rept. & 285 & 160 & 16 & 100 & Elec. & 86 & Water-level measurement on $3 / 8 / 79$ \\
\hline$S B-06-45-28-B D B$ & 402800102231.000 & Mixed & Pivot & 120 & Rept. & 351 & -- & 18 & 100 & Elec. & -- & \\
\hline$S B-08-46-12-B C A$ & 404059102265900 & Corn & Pivot & 130 & Rept. & 288 & 142 & 16 & 100 & Elec. & 64 & Water-level measurement on $4 / 25 / 79$. \\
\hline$S B-08-47-15-D D B$ & 403937102353400 & Corn & Pivot & 140 & Rept. & 262 & 123 & 16 & .110 & N. gas & .147 & Water-level measurement on $4 / 25 / 79$. \\
\hline SB-08-47-22-ACAI & 403910102353601 & Mixed & Pivot & 125 & Meas. & 263 & -- & 16 & 100 & Elec. & -- & \\
\hline$S B-09-43-22-A A C$ & 404446102075700 & Alfalfa & Pivot & 140 & Rept. & 312 & 168 & 16 & 100 & Elec. & -- & Water-level measurement on $4 / 25 / 79$. \\
\hline
\end{tabular}


Table 7b.--Pumpage data for irrigation sites in Phillips County, Colorado

\begin{tabular}{|c|c|c|c|c|c|}
\hline \multirow{2}{*}{$\begin{array}{c}\text { Site } \\
\text { location }\end{array}$} & \multirow{2}{*}{$\begin{array}{l}\text { Field } \\
\text { person }\end{array}$} & \multicolumn{4}{|c|}{ Discharge information } \\
\hline & & Date & $\begin{array}{l}\text { Discharge } \\
\text { (gal/min) }\end{array}$ & Method & Remarks \\
\hline$S B-06-42-07-B A B$ & -- & -- & -- & -- & No discharge measurement made during 1979. \\
\hline SB-06-43-32-CBA & $\begin{array}{l}\text { CEP } \\
\text { RAP }\end{array}$ & $\begin{array}{l}8 / 01 / 79 \\
8 / 01 / 79\end{array}$ & $\begin{array}{r}970 \\
1,000\end{array}$ & $\begin{array}{l}\text { T.T. } \\
\text { In1. }\end{array}$ & $\begin{array}{l}\text { Measurement on } 8 \text {-in steel tubing. } \\
\text { McCrometer inline flow meter. }\end{array}$ \\
\hline SB-06-45-11-DBAI & $\begin{array}{l}\text { JLP } \\
\text { CEP }\end{array}$ & $\begin{array}{l}7 / 25 / 79 \\
7 / 25 / 79\end{array}$ & $\begin{array}{r}1,090 \\
340\end{array}$ & $\begin{array}{l}\text { T.T. } \\
\text { Dop. }\end{array}$ & Measurement on 8-in steel pipe. \\
\hline SB-06-45-28-BDB & -- & -- & -- & -- & No discharge measurement made during 1979. \\
\hline SB-08-46-12-BCA & JLP & $\begin{array}{l}7 / 25 / 79 \\
7 / 25 / 79\end{array}$ & $\begin{array}{l}560 \\
790\end{array}$ & $\begin{array}{l}\text { T.T. } \\
\text { Dop. }\end{array}$ & Measurement on 8 -in steel pipe. \\
\hline SB-08-47-15-DDB & JLP & $\begin{array}{l}7 / 25 / 79 \\
7 / 25 / 79\end{array}$ & $\begin{array}{l}740 \\
730\end{array}$ & $\begin{array}{l}\text { T.T. } \\
\text { Dop. }\end{array}$ & Measurement on 8-in steel pipe. \\
\hline SB-08-47-22-ACA1 & JLP & $\begin{array}{l}8 / 08 / 79 \\
8 / 08 / 79\end{array}$ & $\begin{array}{r}800 \\
1,740\end{array}$ & $\begin{array}{l}\text { T.T. } \\
\text { Dop. }\end{array}$ & Measurement on 8 -in steel pipe. \\
\hline SB-09-43-22-AAC & JLP & $\begin{array}{l}7 / 24 / 79 \\
7 / 24 / 79 \\
7 / 24 / 79\end{array}$ & $\begin{array}{l}930 \\
470 \\
850\end{array}$ & $\begin{array}{l}\text { T.T. } \\
\text { Dop. } \\
\text { Inl. }\end{array}$ & $\begin{array}{l}\text { Measurement on 8-in steel pipe. } \\
\text { McCrometer inline flow meter. }\end{array}$ \\
\hline
\end{tabular}


Table 7c.--Pumpage data for irrigation sites in Phillips County, Colorado

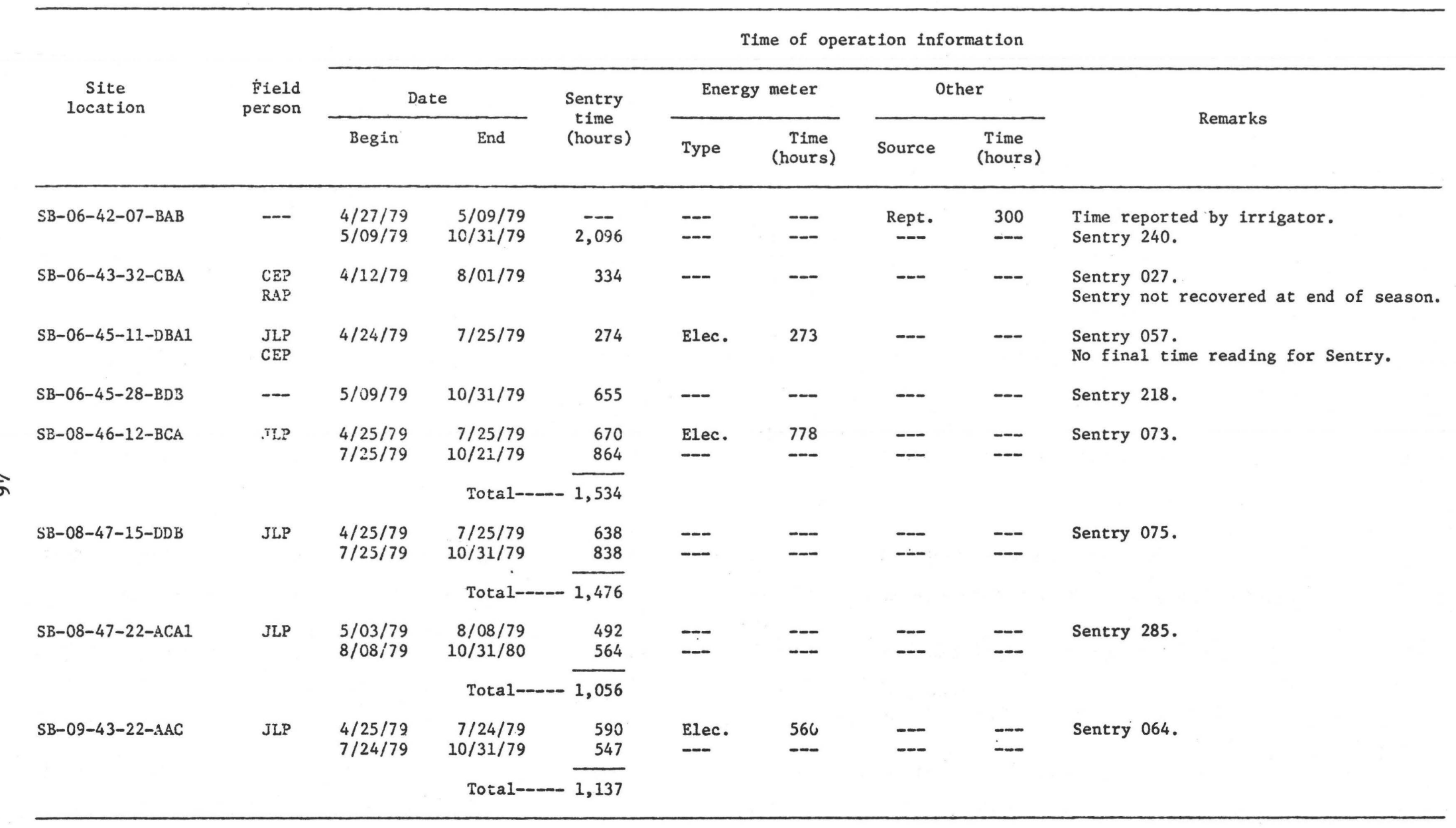


Table 8a.--Pumpare data for irrigation sites in Yuma County, Colorado

\begin{tabular}{|c|c|c|c|c|c|c|c|c|c|c|c|c|}
\hline \multirow{2}{*}{$\begin{array}{c}\text { Site } \\
\text { location }\end{array}$} & \multirow{2}{*}{$\begin{array}{l}\text { Station } \\
\text { identifier }\end{array}$} & \multicolumn{4}{|c|}{ Irrigated crop information } & \multicolumn{6}{|c|}{ Well information } & \multirow[b]{2}{*}{ Remarks } \\
\hline & & Type & System & Acres & Source & $\begin{array}{c}\text { Depth } \\
\text { (ft) }\end{array}$ & $\begin{array}{r}\text { Water } \\
\text { level } \\
(\mathrm{ft})\end{array}$ & $\begin{array}{l}\text { Diam- } \\
\text { meter } \\
\text { (in) }\end{array}$ & $\begin{array}{l}\text { Pump } \\
\text { hp. }\end{array}$ & $\begin{array}{l}\text { Energy } \\
\text { source }\end{array}$ & $\begin{array}{l}\text { Energy } \\
\text { rating }\end{array}$ & \\
\hline SB-01-45-06-ACA & 400504102263000 & Corn & Pivot & 160 & Rept. & 338 & 63 & 16 & 100 & N. gas & -- & Water-level measurement on $2 / 16 / 79$. \\
\hline$S B-01-45-14-A B D$ & 400327102215600 & Corn & Pivot & 135 & Rept. & 270 & 122 & 16 & 100 & N. gas & -- & Water-level measurement on $3 / 2 / 79$ \\
\hline$S B-01-46-25-B B D$ & 400143102281400 & Alfalfa & Pivot & 125 & Est. & 260 & 67 & 16 & 75 & Elec. & -- & Water-leve1 measurement on $5 / 11 / 79$. \\
\hline SB-01-46-29-BBD1 & 400142102323601 & Corn & Pivot & 130 & Rept. & 180 & 77 & 16 & 75 & Elec. & -- & Water-level measurement on $4 / 5 / 79$ \\
\hline SB-01-47-04-CAC & 400450102381000 & Corn & Flood & 177 & Rept. & 314 & 121 & 16 & 70 & N. gas & -- & Water-level measurement on $2 / 28 / 79$. \\
\hline SB-01-47-08-CBD & 400350102393000 & Corn & Pivot & 130 & Rept. & 312 & 139 & 16 & 125 & N. gas & 0.22 & Water-level measurement on $5 / 4 / 79$. \\
\hline SB-02-43-10-ADAI & 400929102090601 & Corn & Pivot & 130 & Rept. & 242 & 53 & 16 & 75 & Elec. & 68 & Water-level measurement on $3 / 1 / 79$. \\
\hline SB-02-46-26-DBD & 400640102283000 & Corn & Pivot & 127 & Meas. & 300 & -- & 16 & 150 & N. gas & -- & \\
\hline$S B-02-47-09-A D C$ & 400925102375000 & Corn & Pivot & 135 & Meas. & 335 & 133 & 16 & 125 & N. gas & -- & Water-level measurement on $2 / 28 / 79$. \\
\hline$S B-02-47-10-A D B$ & 400940102363000 & Corn & Pivot & 130 & Rept. & 360 & 121 & 22 & 100 & Elec. & -- & Water-level measurement on $4 / 4 / 79$ \\
\hline SB-03-43-32-AABI & 401128102113801 & Alfalfa & Pivot & 130 & Meas. & 326 & -- & 16 & 100 & Elec. & 71 & \\
\hline
\end{tabular}


Table 8b.--Pumpage data for irrigation sites in Yuma County, Colorado

\begin{tabular}{|c|c|c|c|c|c|}
\hline \multirow{2}{*}{$\begin{array}{l}\text { Site } \\
\text { location }\end{array}$} & \multirow{2}{*}{$\begin{array}{r}\text { Field } \\
\text { nerson }\end{array}$} & \multicolumn{4}{|c|}{ Discharge information } \\
\hline & & Date & $\begin{array}{l}\text { Discharge } \\
\text { (gal/min) }\end{array}$ & Method & Remarks \\
\hline SB-01-45-06-ACA & $\begin{array}{l}\text { CEP } \\
\text { RAP }\end{array}$ & $\begin{array}{l}8 / 08 / 79 \\
8 / 08 / 79\end{array}$ & $\begin{array}{l}480 \\
620\end{array}$ & $\begin{array}{l}\text { T. T. } \\
\text { Inl. }\end{array}$ & $\begin{array}{l}\text { Measurement on } 8 \text {-in steel pipe. Engine vibrations } \\
\text { present. Type of meter not noted. }\end{array}$ \\
\hline SB-01-45-14-ABD & CEP & $9 / 07 / 79$ & 810 & T.T. & Measurement on 8 -in steel tubing. \\
\hline$S B-01-46-25-B B D$ & -- & --- & -- & -- & No discharge measurement made during 1979. \\
\hline SB-01-46-29-BBD1 & JLP & $\begin{array}{l}8 / 03 / 79 \\
8 / 03 / 79\end{array}$ & $\begin{array}{r}1,140 \\
630\end{array}$ & $\begin{array}{l}\text { T.T. } \\
\text { Dop. }\end{array}$ & Measurement on 8-in steel pipe. \\
\hline SB-01-47-04-CAC & JLP & $\begin{array}{l}8 / 01 / 79 \\
8 / 01 / 79\end{array}$ & $\begin{array}{l}1,500 \\
1,190\end{array}$ & $\begin{array}{l}\text { T.T. } \\
\text { Prop. }\end{array}$ & $\begin{array}{l}\text { Measurement on 10-in aluminum-gated pipe. Estimated the } \\
\text { slope correction to be } 1.1 \text {. }\end{array}$ \\
\hline$S B-01-47-08-C B D$ & JLP & $\begin{array}{l}8 / 06 / 79 \\
8 / 06 / 79\end{array}$ & $\begin{array}{l}670 \\
610\end{array}$ & $\begin{array}{l}\text { T.T. } \\
\text { Dop. }\end{array}$ & M'easurement on 8 -in steel tubing. \\
\hline SB-02-43-10-ADA1 & JLP & $7 / 25 / 79$ & 1,030 & T.T. & Measurement on 8-in steel pipe. \\
\hline $\mathrm{SB}-02-46-26-\mathrm{DBD}$ & $\begin{array}{l}\text { CEP } \\
\text { RAP }\end{array}$ & $\begin{array}{l}8 / 06 / 79 \\
8 / 06 / 79\end{array}$ & $\begin{array}{l}770 \\
730\end{array}$ & $\begin{array}{l}\text { T.T. } \\
\text { R.G. }\end{array}$ & $\begin{array}{l}\text { Measurement on } 8 \text {-in steel pipe. } \\
\text { Average of } 0.73 \mathrm{in.} \text { of water caught in rain gages. }\end{array}$ \\
\hline$S B-02-47-09-A D C$ & CEP & $9 / 05 / 79$ & 1,060 & T.T. & Measurement on 8 -in steel tubing. \\
\hline$S B-02-47-10-A D B$ & CEP & $9 / 05 / 79$ & 1,010 & T.T. & Measurement on 8 -in steel tubing. \\
\hline SB-03-43-32-AABI & JLP & $\begin{array}{l}7 / 25 / 79 \\
7 / 25 / 79\end{array}$ & $\begin{array}{l}1,070 \\
1,030\end{array}$ & $\begin{array}{l}\text { T.T. } \\
\text { Dop. }\end{array}$ & Measurement on 8-in steel pipe. \\
\hline
\end{tabular}


Table 8c.--Pumpage data for irrigation sites in Yuma County, Colorado

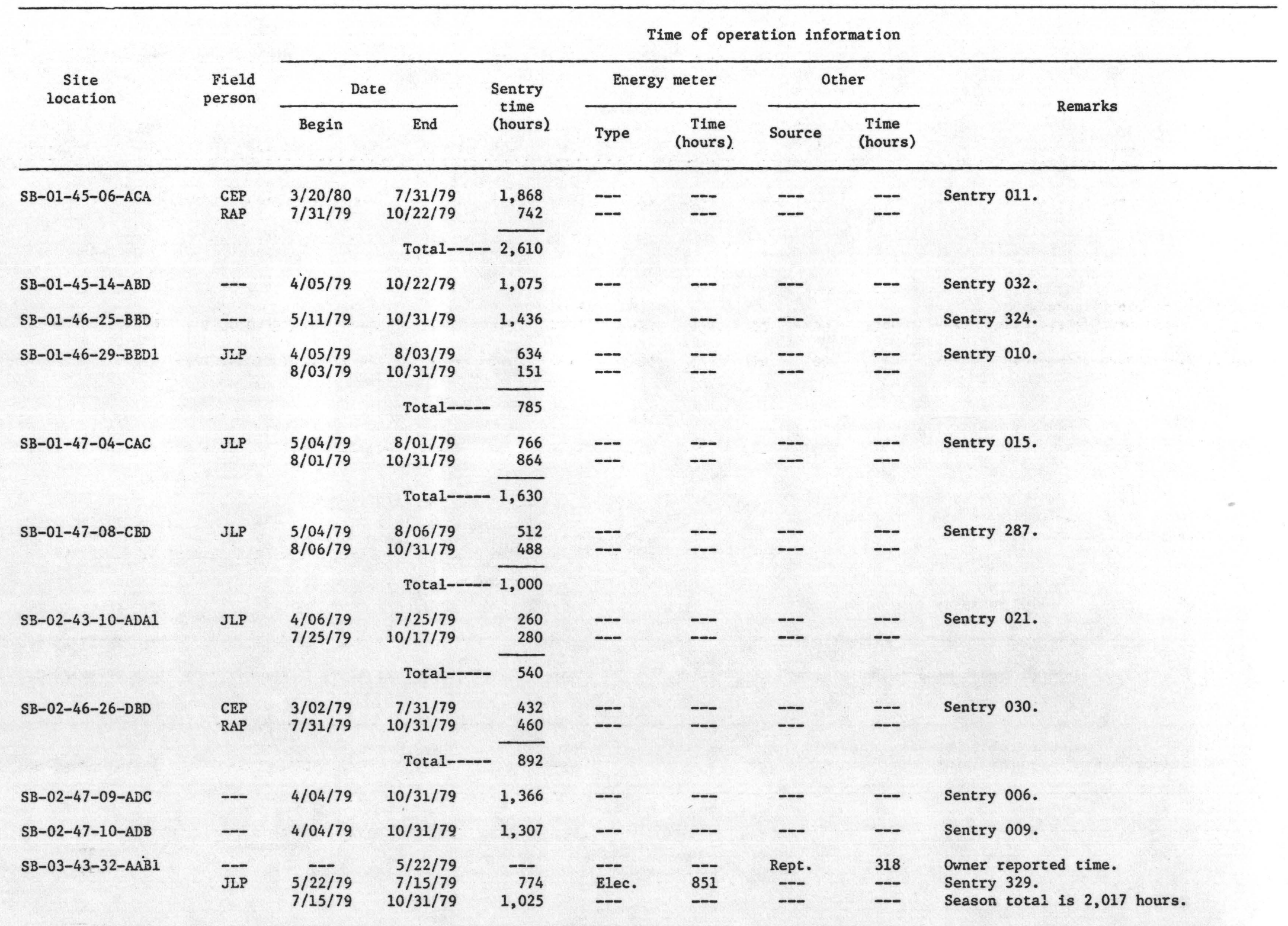


Table 8a--Pumpage data for irrigution sites in Yuma County, Colorado--Cont1nued

\begin{tabular}{|c|c|c|c|c|c|c|c|c|c|c|c|c|}
\hline \multirow{2}{*}{$\begin{array}{c}\text { Site } \\
\text { location }\end{array}$} & \multirow{2}{*}{$\begin{array}{l}\text { Station } \\
\text { identifier }\end{array}$} & \multicolumn{4}{|c|}{ Irrigated crop information } & \multicolumn{6}{|c|}{ Well information } & \multirow[b]{2}{*}{ Remarks } \\
\hline & & Type & System & Acres & Source & $\begin{array}{c}\text { Depth } \\
(f t)\end{array}$ & $\begin{array}{r}\text { Water } \\
\text { level } \\
\text { (ft) }\end{array}$ & $\begin{array}{l}\text { Diam- } \\
\text { meter } \\
\text { (in). }\end{array}$ & $\begin{array}{c}\text { Pump } \\
\text { hp. }\end{array}$ & $\begin{array}{l}\text { Energy } \\
\text { source }\end{array}$ & $\begin{array}{l}\text { Energy } \\
\text { rating }\end{array}$ & \\
\hline $\mathrm{SB}-03-43-33-\mathrm{ACA} 1$ & 401116102103101 & Corn & Pivot & 130 & Rept. & 327 & 43 & 16 & 75 & Elec. & 63 & Water-level measurement on $2 / 15 / 79$. \\
\hline $\mathrm{SB}-03-44-06-\mathrm{ABD} 1$ & 401537102193201 & Alfalfa. & Pivot & 125 & Est. & 300 & 65 & 16 & 100 & Elec. & -- & Water-level measurement on $5 / 11 / 79$. \\
\hline SB-03-44-08-ACA1 & 401445102182401 & Corn & Pivot & 130 & Rept. & 340 & -- & 16 & 100 & Elec. & 71 & \\
\hline $\mathrm{SB}-03-44-15-\mathrm{ACA}$ & 401350102160000 & Corn & Pivot & $\begin{array}{l}130 \\
116\end{array}$ & $\begin{array}{l}\text { Rept. } \\
\text { Meas. }\end{array}$ & 280 & 56 & 16 & 75 & Elec. & 69 & $\begin{array}{l}\text { Water-level measurement on } 4 / 12 / 79 \text {. } \\
\text { Acreage calculated using vehicle } \\
\text { odometer. }\end{array}$ \\
\hline $\mathrm{SB}-03-44-23-\mathrm{ACA}$ & 401310102150000 & Alfalfa & Pivot & 200 & Rept. & 325 & 47 & 16 & 100 & Elec. & -- & Water-level measurement on $5 / 10 / 79$. \\
\hline$S B-03-46-15-C A C$ & 401340102301000 & Corn & Pivot & 130 & Est. & 384 & 112 & 16 & 100 & Elec. & -- & Water-level measurement on $3 / 20 / 79$. \\
\hline$S B-03-46-18-D B D$ & 401330102331000 & Corn & Pivot & $\begin{array}{l}140 \\
116\end{array}$ & $\begin{array}{l}\text { Rept. } \\
\text { Meas. }\end{array}$ & 380 & 122 & 22 & 100 & Elec. & -- & $\begin{array}{l}\text { Water-level measurement on } 5 / 4 / 79 \text {. } \\
\text { Acreage calculated using vehicle } \\
\text { odometer. }\end{array}$ \\
\hline$S B-03-47-19-C D B$ & 401240102403000 & Corn & Pivot & 260 & Rept. & 293 & 164 & -- & -- & N. gas & -- & Water-level measurement on $2 / 26 / 79$. \\
\hline $\mathrm{SB}-03-47-25-\mathrm{AAC}$ & $401210 i 02341000$ & Corn & Pivot & 130 & Est. & 345 & 124 & 22 & 100 & Elec. & 76 & Water-level measurement on $4 / 5 / 79$ \\
\hline$S B-03-48-21-C B D$ & 401230102450000 & Corn & Pivot & 130 & Rept. & 328 & 190 & 16 & 110 & N. gas & -- & Water-level measurement on $2 / 26 / 79$. \\
\hline
\end{tabular}


Table 8b--Pumpage data for imrigation sites in Yuma County, Colorado--Continued

\begin{tabular}{|c|c|c|c|c|c|}
\hline \multirow{2}{*}{$\begin{array}{c}\text { Site } \\
\text { location }\end{array}$} & \multirow{2}{*}{$\begin{array}{r}\text { Field } \\
\text { nerson }\end{array}$} & \multicolumn{4}{|c|}{ Discharge information } \\
\hline & & Date & $\begin{array}{l}\text { Discharge } \\
\text { (gal/min) }\end{array}$ & Method & Remarks \\
\hline SB-03-43-33-ACA1 & JLP & $7 / 25 / 72$ & 1,180 & T.T. & Measurement on 8 -in steel tubing. \\
\hline$S P-03-44-06-A B D 1$ & -- & -- & -- & -- & No discharge measurement made during 1979. \\
\hline$S B-03-44-08-A C A 1$ & CEP & $8 / 08 / 79$ & 620 & T.T. & Measurement on 8-in steel tubing. \\
\hline$S B-03-44-15-A C A$ & $\begin{array}{l}\text { CEP } \\
\text { KRW }\end{array}$ & $\begin{array}{l}8 / 08 / 79 \\
8 / 23 / 79\end{array}$ & $\begin{array}{l}570 \\
990\end{array}$ & $\begin{array}{l}\text { T.T. } \\
\text { T.T. }\end{array}$ & Measurement on 8 -in steel tubing. \\
\hline$S B-03-44-23-A C A$ & JLP & $7 / 25 / 79$ & 1,090 & T.T. & Measurement on 8-in steel tubing. \\
\hline $\mathrm{SB}-03-46-15-\mathrm{CAC}$ & - & -- & -- & -- & No discharge measurement made during 1979. \\
\hline$S B-03-46-18-D B D$ & $\begin{array}{l}\text { CEP } \\
\text { JLP } \\
\text { CEP } \\
\text { RRL }\end{array}$ & $\begin{array}{l}7 / 31 / 79 \\
8 / 01 / 79 \\
8 / 01 / 79 \\
8 / 18 / 79\end{array}$ & $\begin{array}{l}820 \\
920 \\
340 \\
550\end{array}$ & $\begin{array}{l}\text { T.T. } \\
\text { T.T. } \\
\text { Dop. } \\
-\end{array}$ & Measurement on 8-in steel tubing. \\
\hline$S B-03-47-19-C D B$ & CEP & $\begin{array}{l}7 / 31 / 79 \\
7 / 31 / 79\end{array}$ & $\begin{array}{l}1,020 \\
1,460\end{array}$ & $\begin{array}{l}\text { T.T. } \\
\text { Inl. }\end{array}$ & $\begin{array}{l}\text { Combined discharge of two pivots that were measured } \\
\text { separately. }\end{array}$ \\
\hline$S B-03-47-25-A A C$ & $\begin{array}{l}\text { CEP } \\
\text { RAP }\end{array}$ & $7 / 31 / 79$ & 830 & T.T. & Measurement on 8-in steel tubing. \\
\hline$S B-03-48-21-C B D$ & $\begin{array}{l}\text { CEP } \\
\text { RAP }\end{array}$ & $7 / 31 / 79$ & 580 & T.T. & Measurement on 8-in steel tubing. \\
\hline
\end{tabular}


Table 8c--Pumpage data for irrigation sites in Ywma County, Colorado--Continued

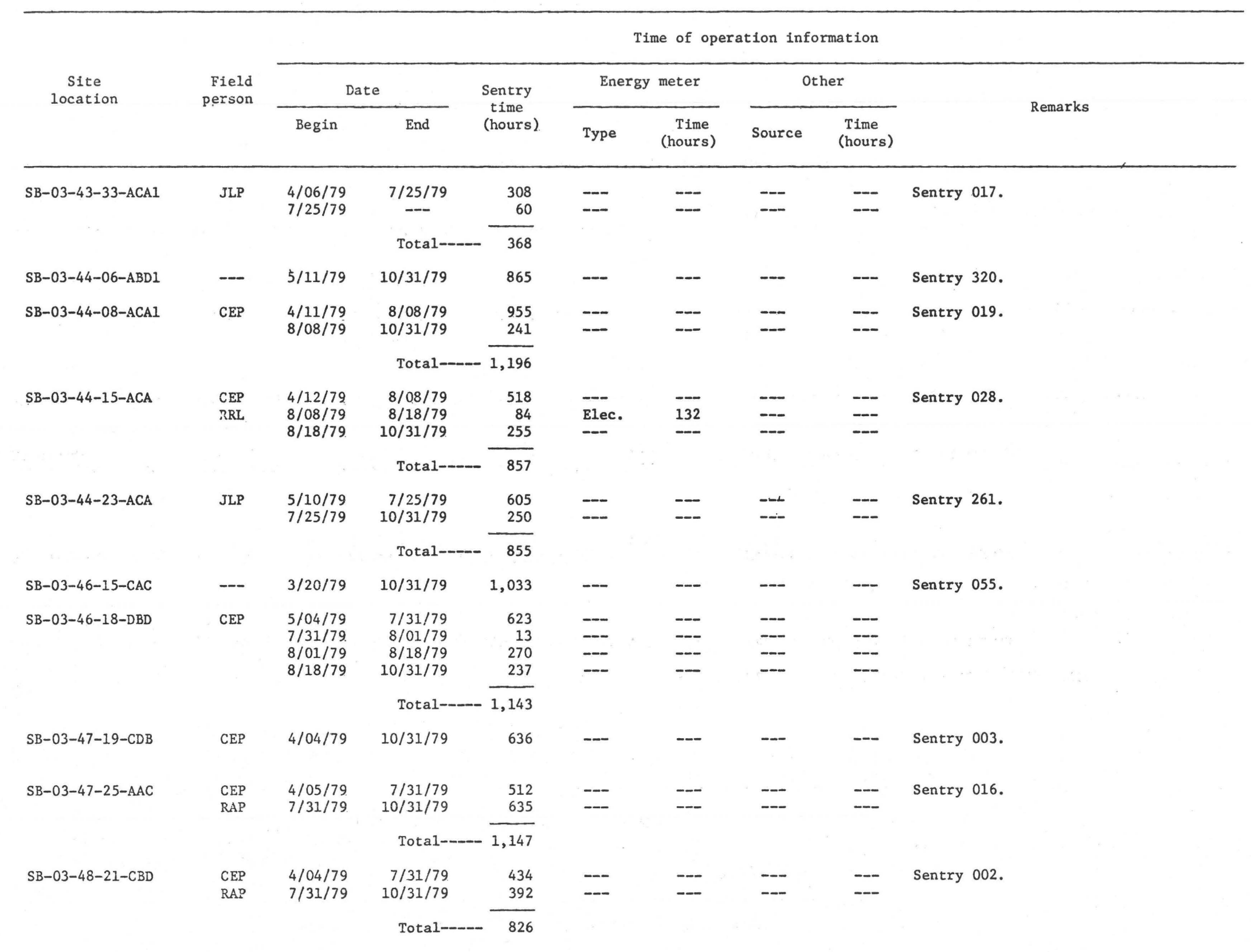


Table 8a.--Pumpage data for irrigation sites in Yuma County, Colorado--Continued

\begin{tabular}{|c|c|c|c|c|c|c|c|c|c|c|c|c|}
\hline \multirow{2}{*}{$\begin{array}{c}\text { Site } \\
\text { location }\end{array}$} & \multirow{2}{*}{$\begin{array}{l}\text { Station } \\
\text { identifier }\end{array}$} & \multicolumn{4}{|c|}{ Irrigated crop information } & \multicolumn{6}{|c|}{ Well information } & \multirow[b]{2}{*}{ Remarks } \\
\hline & & Type & System & Acres & Source & $\begin{array}{r}\text { Depth } \\
(\mathrm{ft})\end{array}$ & $\begin{array}{r}\text { Water } \\
\text { leve1 } \\
(\mathrm{ft})\end{array}$ & $\begin{array}{l}\text { Diam- } \\
\text { meter } \\
\text { (in) }\end{array}$ & $\begin{array}{l}\text { Pump } \\
\text { hp. }\end{array}$ & $\begin{array}{l}\text { Energy } \\
\text { source }\end{array}$ & $\begin{array}{l}\text { Energy } \\
\text { rating }\end{array}$ & \\
\hline$S B-04-43-05-A A D 1$ & 402052102111801 & Corn & Pivot & 240 & Rept. & 345 & 65 & 16 & 125 & Elec. & 116.8 & Water-level measurement on $4 / 13 / 79$. \\
\hline SB-04-43-09-DBD1 & 401933102102801 & Alfalfa & Pivot & 90 & Rept. & 330 & 61 & 10 & 100 & Elec. & -- & Water-level measurement on $5 / 10 / 79$. \\
\hline$S B-04-43-26-D D B$ & 401705102081000 & Corn & Pivot & 170 & Rept. & 380 & 17 & 22 & 100 & Elec. & -- & Water-level measurement on $7 / 24 / 69$. \\
\hline$S B-04-43-27-A D B$ & 401730102092000 & Corn & Pivot & 185 & Rept. & 365 & 40 & 22 & 100 & Elec. & -- & Water-level measurement on $6 / 13 / 69$. \\
\hline $\mathrm{SB}-04-43-29-\mathrm{AACl}$ & 401721102113101 & $\begin{array}{l}\text { Alfalfa } \\
\text { Pasture }\end{array}$ & Pivot & $\begin{array}{l}65 \\
65\end{array}$ & Rept. & 305 & 42 & 16 & -- & Diesel & - & Water-level measurement on $5 / 3 / 79$ \\
\hline $\mathrm{SB}-04-44-35-\mathrm{ACA}$ & 401630102150000 & $\begin{array}{l}\text { Corn } \\
\text { Pasture }\end{array}$ & $\begin{array}{l}\text { Sprink- } \\
\text { ler }\end{array}$ & $\begin{array}{l}75 \\
75\end{array}$ & Rept. & 355 & 63 & 16 & 100 & Elec. & 90.9 & Water-level measurement on $5 / 5 / 79$ \\
\hline $\mathrm{SB}-04-45-18-\mathrm{AACl}$ & 401914102261601 & Alfalfa & Pivot & 130 & Rept. & 290 & 129 & 16 & 100 & Elec. & -- & Water-level measurement on $11 / 9 / 74$. \\
\hline SB-04-46-33-DCA1 & 401607102305001 & Corn & $\begin{array}{l}\text { Pivot } \\
\text { Flood }\end{array}$ & $\begin{array}{l}130 \\
150\end{array}$ & Rept. & 410 & 130 & 16 & -- & N. gas & -- & Water-level measurement on $4 / 9 / 79$ \\
\hline
\end{tabular}


Table 8b.--Pumpage data for irrigation sites in Yuma County, Colorado--Continued

\begin{tabular}{|c|c|c|c|c|c|}
\hline \multirow{2}{*}{$\begin{array}{c}\text { Site } \\
\text { location }\end{array}$} & \multirow{2}{*}{$\begin{array}{r}\text { Field } \\
\text { person }\end{array}$} & \multicolumn{4}{|c|}{ Discharge information } \\
\hline & & Date & $\begin{array}{l}\text { Discharge } \\
\text { (gal/min) }\end{array}$ & Method & Remarks \\
\hline$S B-04-43-05-A A D 1$ & JLP & $\begin{array}{l}8 / 08 / 79 \\
8 / 08 / 79\end{array}$ & $\begin{array}{r}1,585 \\
510\end{array}$ & $\begin{array}{l}\text { T.T. } \\
\text { Dop. }\end{array}$ & Measurement on 8-in steel pipe. \\
\hline SB-04-43-09-DBD1 & JLP & $\begin{array}{l}8 / 08 / 79 \\
8 / 08 / 79\end{array}$ & $\begin{array}{r}1,130 \\
380\end{array}$ & $\begin{array}{l}\text { T.T. } \\
\text { Dop. }\end{array}$ & Measurement on 8-in steel pipe. \\
\hline$S B-04-43-26-D D B$ & CEP & $8 / 08 / 79$ & 715 & T.T. & Measurement on 8 -in steel tubing. \\
\hline$S B-04-43-27-A D B$ & CEP & $\begin{array}{l}8 / 08 / 79 \\
8 / 08 / 79\end{array}$ & $\begin{array}{l}790 \\
970\end{array}$ & $\begin{array}{l}\text { T.T. } \\
\text { InI. }\end{array}$ & Measurement on 8-in steel pipe. \\
\hline SB-04-43-29-AAC1 & CEP & $8 / 08 / 79$ & 790 & T.T. & Measurement on 8-in steel tubing. \\
\hline $\mathrm{SB}-04-44-35-\mathrm{ACA}$ & $\begin{array}{l}\text { CEP } \\
\text { RAP }\end{array}$ & $8 / 01 / 79$ & 1,065 & T.T. & Measurement on 8-in steel pipe. \\
\hline SB-04-45-18-AAC1 & JLP & $\begin{array}{l}8 / 06 / 79 \\
8 / 06 / 79\end{array}$ & $\begin{array}{l}960 \\
550\end{array}$ & $\begin{array}{l}\text { T.T. } \\
\text { Dop. }\end{array}$ & Measurement on 8 -in steel tubing. \\
\hline$S B-04-46-33-D C A 1$ & $\begin{array}{l}\mathrm{KRW} \\
\mathrm{KRW}\end{array}$ & $\begin{array}{l}8 / 22 / 79 \\
8 / 23 / 79\end{array}$ & $\begin{array}{l}650 \\
720\end{array}$ & $\begin{array}{l}\text { Buck } \\
\text { T.T. }\end{array}$ & $\begin{array}{l}\text { Measurement on } 8 \text {-in aluminum-gated pipe. } \\
\text { Measurement on } 8 \text {-in steel tubing for pivot. }\end{array}$ \\
\hline
\end{tabular}


Table 8c.--Pumpage data for irrigation sites in Yuma County, Colorado--Continued

\begin{tabular}{|c|c|c|c|c|c|c|c|c|c|}
\hline \multirow{3}{*}{$\begin{array}{l}\text { Site } \\
\text { location }\end{array}$} & \multirow{3}{*}{$\begin{array}{r}\text { Field } \\
\text { person }\end{array}$} & \multicolumn{8}{|c|}{ Time of operation information } \\
\hline & & \multicolumn{2}{|c|}{ Date } & \multirow{2}{*}{$\begin{array}{l}\text { Sentry } \\
\text { time } \\
\text { (hours) }\end{array}$} & \multicolumn{2}{|c|}{ Energy meter } & \multicolumn{2}{|c|}{ Other } & \multirow{2}{*}{ Remarks } \\
\hline & & Begin & End & & Type & $\begin{array}{c}\text { Time } \\
\text { (hours) }\end{array}$ & Source & $\begin{array}{l}\text { Time } \\
\text { (hours) }\end{array}$ & \\
\hline \multirow[t]{2}{*}{$\mathrm{SB}-04-43-05-\mathrm{AAD} 1$} & JLP & $\begin{array}{l}4 / 13 / 79 \\
8 / 08 / 79\end{array}$ & $\begin{array}{r}8 / 08 / 79 \\
10 / 31 / 79\end{array}$ & $\begin{array}{l}695 \\
243\end{array}$ & Elec. & 767 & -- & -- & Sentry 033. \\
\hline & & & Totai- & 938 & & & & & \\
\hline \multirow[t]{2}{*}{$S B-04-43-09-D B D 1$} & JLP & $\begin{array}{l}-- \\
5 / 10 / 79 \\
8 / 08 / 79\end{array}$ & $\begin{array}{r}5 / 10 / 79 \\
8 / 08 / 79 \\
10 / 31 / 79\end{array}$ & $\begin{array}{l}703 \\
464\end{array}$ & \begin{tabular}{c} 
Elec. \\
\hdashline- \\
--
\end{tabular} & -- & $\begin{array}{c}\text { Farmer } \\
--\end{array}$ & $\begin{array}{r}48 \\
-- \\
--\end{array}$ & Sentry 295. \\
\hline & & & Total- & $-1,167$ & -- & -- & -- & -- & Season total is 1,215 hours. \\
\hline \multirow[t]{2}{*}{$S B-04-43-26-D D B$} & CEP & $\begin{array}{l}4 / 13 / 79 \\
8 / 08 / 79\end{array}$ & $\begin{array}{r}8 / 08 / 79 \\
10 / 31 / 79\end{array}$ & $\begin{array}{r}166 \\
11 \\
\end{array}$ & Elec. & -- & -- & $\cdots$ & $\begin{array}{l}\text { Sentry } .029 \\
\text { Time seems very low. }\end{array}$ \\
\hline & & & Total- - & 177 & & & & & \\
\hline \multirow[t]{2}{*}{$S B-04-43-27-A D B$} & CEP & $\begin{array}{l}4 / 13 / 79 \\
8 / 08 / 79\end{array}$ & $\begin{array}{r}8 / 08 / 79 \\
10 / 23 / 79\end{array}$ & $\begin{array}{r}178 \\
53 \\
\end{array}$ & Elec. & -- & -- & $\cdots$ & $\begin{array}{l}\text { Sentry } 031 \text {. } \\
\text { Time seems very low. }\end{array}$ \\
\hline & & & Tota1- - & 231 & & & & & \\
\hline SB-04-43-29-AACl & CEP & $\begin{array}{l}4 / 27 / 79 \\
8 / 08 / 79\end{array}$ & $\begin{array}{r}8 / 08 / 79 \\
10 / 22 / 79\end{array}$ & $\begin{array}{l}584 \\
715 \\
\end{array}$ & $\overline{--}$ & -- & -- & -- & Sentry 216 \\
\hline & & & Total-- - & $-1,299$ & & & & & \\
\hline $\mathrm{SB}-04-44-35-\mathrm{ACA}$ & $\begin{array}{l}\text { CEP } \\
\text { RAP }\end{array}$ & $\begin{array}{l}5 / 03 / 79 \\
8 / 01 / 79\end{array}$ & $\begin{array}{r}8 / 01 / 79 \\
10 / 31 / 79\end{array}$ & $\begin{array}{l}686 \\
604 \\
\end{array}$ & - & -- & -- & -- & Sentry 301. \\
\hline & & & Total- - & $-1,290$ & & & & & \\
\hline $\mathrm{SB}-04-45-18-\mathrm{AACl}$ & JLP & $\begin{array}{l}4 / 27 / 79 \\
8 / 06 / 79\end{array}$ & $\begin{array}{r}8 / 06 / 79 \\
10 / 31 / 79\end{array}$ & $\begin{array}{r}1,083 \\
353 \\
\end{array}$ & - & -- & - & $\cdots$ & Sentry 281. \\
\hline & & & Total- - & $-1,436$ & & & & & \\
\hline $\mathrm{SB}-04-46-33-\mathrm{DCAI}$ & KRW & $\begin{array}{l}5 / 18 / 79 \\
8 / 22 / 79\end{array}$ & $\begin{array}{l}8 / 22 / 79 \\
9 / 20 / 79\end{array}$ & $\begin{array}{r}1,193 \\
494\end{array}$ & N. gas & 1,203 & $\overline{--}$ & - & Sentry 307. \\
\hline
\end{tabular}


Table 8a.--Pumpage data for irrigation sites in Yuma County, Colorado--Continued

\begin{tabular}{|c|c|c|c|c|c|c|c|c|c|c|c|c|}
\hline \multirow{2}{*}{$\begin{array}{c}\text { Site } \\
\text { location }\end{array}$} & \multirow{2}{*}{$\begin{array}{l}\text { Station } \\
\text { identifier }\end{array}$} & \multicolumn{4}{|c|}{ Irrigated crop information } & \multicolumn{6}{|c|}{ We11 information } & \multirow{2}{*}{ Remarks } \\
\hline & & Type & System & Acres & Source & $\begin{array}{l}\text { Depth } \\
\text { (ft) }\end{array}$ & $\begin{array}{r}\text { Water } \\
\text { level } \\
(\mathrm{ft})\end{array}$ & $\begin{array}{l}\text { Diam- } \\
\text { meter } \\
\text { (in) }\end{array}$ & $\begin{array}{c}\text { Pump } \\
\text { hp. }\end{array}$ & $\begin{array}{l}\text { Energy } \\
\text { source }\end{array}$ & $\begin{array}{l}\text { Energy } \\
\text { rating }\end{array}$ & \\
\hline SB-05-42-06-CDBI & 402543102050301 & Corn & Pivot & 130 & Rept. & 349 & 36 & 16 & 60 & Elec. & 55.7 & Water-level measuremént on $4 / 12 / 79$. \\
\hline $\mathrm{SB}-05-42-18-\mathrm{BBD} 1$ & 402428102050501 & Corn & Pivot & 130 & Rept. & 325 & 58 & 16 & 75 & E1ec. & 54.4 & Water-level measurement on $2 / 14 / 79$. \\
\hline $\mathrm{SB}-05-42-32-\mathrm{BCA}$ & 402150102041000 & Corn & Pivot & 130 & Rept. & 300 & 41 & 12.5 & 60 & Elec. & 48.22 & Water-level measurement on $2 / 14 / 79$. \\
\hline$S B-05-43-36-A C A$ & 402140102054000 & Corn & Pivot & 130 & Rept. & 333 & 33 & 18 & 75 & Elec. & 59.6 & Water-level measurement on $5 / 3 / 79$ \\
\hline$S B-05-44-29-D D C 1$ & $؛ 02204102170101$ & Alfalfa & Pivot & 220 & Rept. & 355 & 95 & 16 & 200 & Elec. & -- & Water-level measurement on $4 / 26 / 76$. \\
\hline$S B-05-44-35-D A C 1$ & 402121102133801 & Grass & Pivot & 94 & Rept. & 304 & 92 & 16 & 100 & Elec. & 81.5 & Water-level measurement on $4 / 11 / 79$. \\
\hline$S B-05-45-23-C A B 1$ & 402313102210401 & Corn & Pivot & 311 & Rept. & 370 & 120 & 16 & 200 & Elec. & -- & Water-level measurement on $3 / 24 / 77$. \\
\hline SB-05-45-36-AAC1 & 402152102192201 & Corn & Pivot & 130 & Rept. & 290 & 107 & 16 & 125 & Elec. & -- & Water-level masurement on $2 / 15 / 79$. \\
\hline$S B-05-46-21-A D C$ & 402310102294500 & Alfaifa & Pivot & 260 & Rept. & 386 & 161 & 16 & 125 & Elec. & -- & Water-level measurement on $9 / 26 / 70$. \\
\hline
\end{tabular}


Table 8b.--Pumpage data for irrigation sites in Yuma County, Colorado--Continued

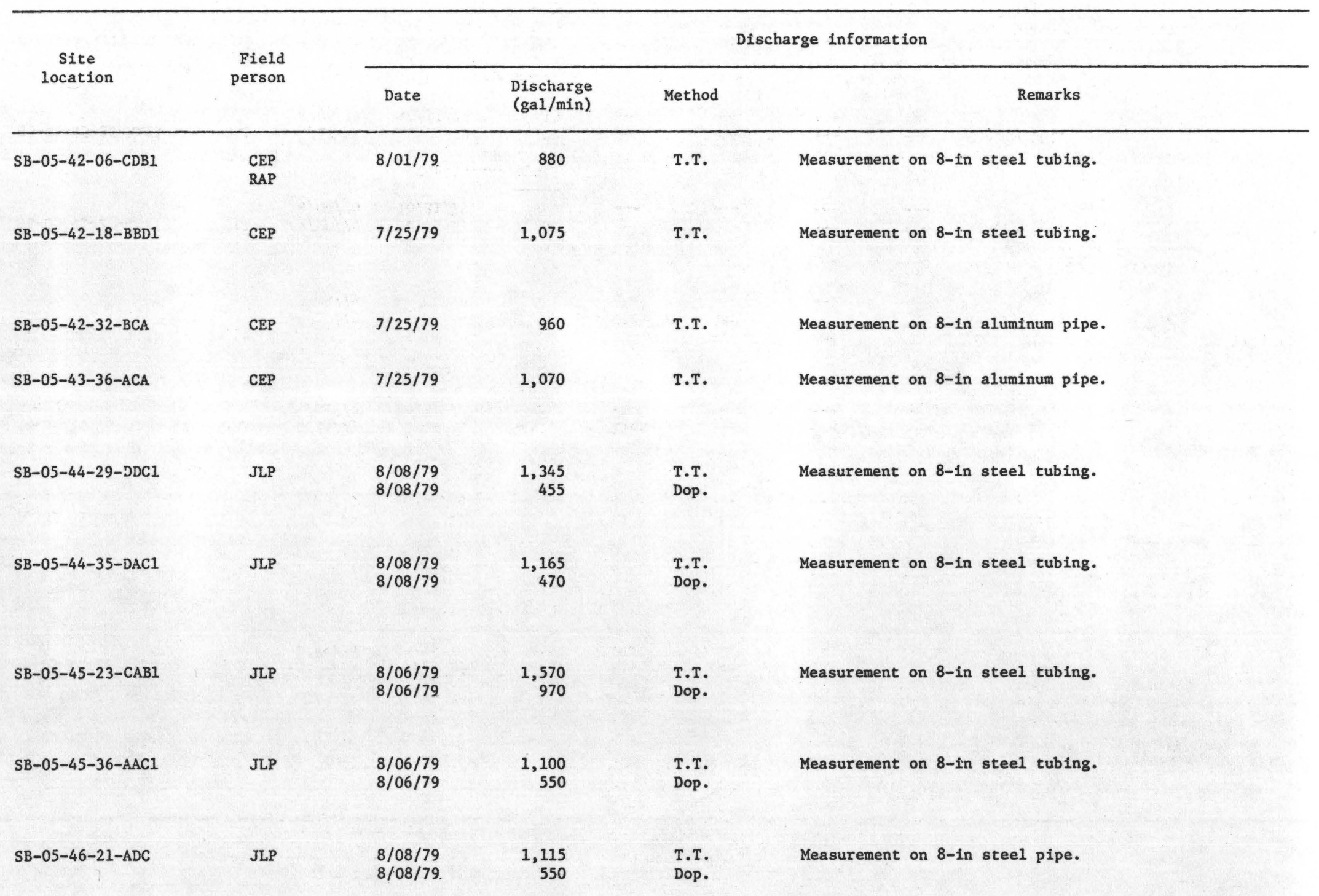


Table 8c.--Pumpage data for irrigation sites in Yuma County, Colorado--Continued

\begin{tabular}{|c|c|c|c|c|c|c|c|c|c|}
\hline \multirow{3}{*}{$\begin{array}{c}\text { Site } \\
\text { location }\end{array}$} & \multirow{3}{*}{$\begin{array}{r}\text { Field } \\
\text { person }\end{array}$} & \multicolumn{8}{|c|}{ Time of operation information } \\
\hline & & \multicolumn{2}{|c|}{ Date } & \multirow{2}{*}{$\begin{array}{l}\text { Sentry } \\
\text { time } \\
\text { (hours) }\end{array}$} & \multicolumn{2}{|c|}{ Energy meter } & \multicolumn{2}{|c|}{ Other } & \multirow{2}{*}{ Remarks } \\
\hline & & Begin & End & & Type & $\begin{array}{c}\text { Time } \\
\text { (hours) }\end{array}$ & Source & $\begin{array}{l}\text { Time } \\
\text { (hours) }\end{array}$ & \\
\hline$S B-05-42-06-C D B 1$ & $\begin{array}{l}\text { CEP } \\
\text { RAP }\end{array}$ & $\begin{array}{l}4 / 12 / 79 \\
8 / 01 / 79\end{array}$ & $\begin{array}{r}8 / 01 / 79 \\
10 / 31 / 79\end{array}$ & --- & -- & --- & --- & -- & $\begin{array}{l}\text { Sentry } 025 \text { found removed from pump on } \\
8 / 1 / 79 ; 670 \text { hours logged from } 4 / 12 / 79 \\
\text { to date that sentry was removed. }\end{array}$ \\
\hline \multirow[t]{2}{*}{$\mathrm{SB}-05-42-18-\mathrm{BBDI}$} & CEP & $\begin{array}{l}4 / 12 / 79 \\
7 / 25 / 79\end{array}$ & $\begin{array}{r}7 / 25 / 79 \\
10 / 31 / 79\end{array}$ & $\begin{array}{l}644 \\
696 \\
\end{array}$ & Elec. & 682 & --- & -- & Sentry 024 \\
\hline & & & Tota1-- & $-1,340$ & & & & & \\
\hline $\mathrm{SB}-05-42-32-\mathrm{BCA}$ & CEP & $\begin{array}{l}4 / 12 / 79 \\
7 / 25 / 79\end{array}$ & $\begin{array}{r}7 / 25 / 79 \\
10 / 31 / 79\end{array}$ & 97.5 & Elec. & 265 & -- & -- & Sentry 022. Sentry bad; not replaced. \\
\hline \multirow[t]{2}{*}{$S B-05-43-36-A C A$} & CEP & $\begin{array}{l}5 / 03 / 79 \\
7 / 25 / 79\end{array}$ & $\begin{array}{r}7 / 25 / 79 \\
10 / 31 / 79\end{array}$ & $\begin{array}{l}581 \\
667 \\
\end{array}$ & Elec. & $\begin{array}{l}587 \\
---\end{array}$ & -- & -- & Sentry 283 . \\
\hline & & & Total-- & 1,248 & & & & & \\
\hline \multirow[t]{2}{*}{$S B-05-44-29-D D C 1$} & JLP & $\begin{array}{l}4 / 26 / 79 \\
8 / 08 / 79\end{array}$ & $\begin{array}{r}8 / 08 / 79 \\
10 / 31 / 79\end{array}$ & $\begin{array}{l}975 \\
325 \\
\end{array}$ & Elec. & -- & -- & -- & Sentry 213. \\
\hline & & & Totai-- & $-1,300$ & & & & & \\
\hline \multirow[t]{2}{*}{$S B-05-44-35-D A C 1$} & JLP & $\begin{array}{l}-\overline{11 / 79} \\
8 / 08 / 79\end{array}$ & $\begin{array}{r}5 / 11 / 79 \\
8 / 08 / 79 \\
10 / 31 / 79\end{array}$ & $\begin{array}{r}--- \\
210 \\
\end{array}$ & Elec. & $\overline{486}$ & $\begin{array}{c}\text { Farmer } \\
--- \\
--\end{array}$ & -- & $\begin{array}{l}\text { Sentry } 316 . \\
\text { Sentry time appears low. }\end{array}$ \\
\hline & & & Total & 617 & & & & & Season total 652 hours. \\
\hline \multirow[t]{2}{*}{$S B-05-45-23-C A B 1$} & JLP & $\begin{array}{l}4 / 26 / 79 \\
8 / 06 / 79\end{array}$ & $\begin{array}{r}8 / 06 / 79 \\
10 / 31 / 79\end{array}$ & $\begin{array}{r}1,051 \\
864 \\
\end{array}$ & $-\cdots$ & -- & -- & -- & Sentry 202 \\
\hline & & & Tota1-- & $-1,915$ & & & & & \\
\hline \multirow[t]{2}{*}{$\mathrm{SB}-05-45-36-\mathrm{AACl}$} & JLP & $\begin{array}{l}4 / 11 / 79 \\
8 / 06 / 79\end{array}$ & $\begin{array}{r}8 / 06 / 79 \\
10 / 31 / 79\end{array}$ & $\begin{array}{r}1,120 \\
415 \\
\end{array}$ & -- & -- & -- & -- & Sentry 001 \\
\hline & & & Total-- & $-1,535$ & & & & & \\
\hline \multirow[t]{2}{*}{$S B-05-46-21-A D C$} & JLP & $\begin{array}{l}4 / 27 / 79 \\
8 / 08 / 79\end{array}$ & $\begin{array}{r}8 / 08 / 79 \\
10 / 31 / 79\end{array}$ & $\begin{array}{l}402 \\
159 \\
\end{array}$ & -- & -- & -- & --- & $\begin{array}{l}\text { Sentry } 230 . \\
\text { Sentry time seems very low. }\end{array}$ \\
\hline & & & Total-- & 561 & & & & & \\
\hline
\end{tabular}


Table 8a.--Pumpage data for irrigation sites in Yuma County, Colorado--Continued

\begin{tabular}{|c|c|c|c|c|c|c|c|c|c|c|c|c|}
\hline \multirow{2}{*}{$\begin{array}{l}\text { Site } \\
\text { location }\end{array}$} & \multirow{2}{*}{$\begin{array}{l}\text { Station } \\
\text { identifier }\end{array}$} & \multicolumn{4}{|c|}{ Irrigated crop information } & \multicolumn{6}{|c|}{ Well information } & \multirow[b]{2}{*}{ Remarks } \\
\hline & & Type & System & Acres & Source & $\begin{array}{c}\text { Depth } \\
(\mathrm{ft})\end{array}$ & $\begin{array}{r}\text { Water } \\
\text { level } \\
(f t)\end{array}$ & $\begin{array}{l}\text { Diam- } \\
\text { meter } \\
\text { (in) }\end{array}$ & $\begin{array}{l}\text { Pump } \\
\text { hp. }\end{array}$ & $\begin{array}{l}\text { Energy } \\
\text { source }\end{array}$ & $\begin{array}{l}\text { Energy } \\
\text { rating }\end{array}$ & \\
\hline$S B-05-46-34-D B C$ & 402120102290000 & Corn & $\begin{array}{l}\text { Pivot } \\
\text { Flood }\end{array}$ & $\begin{array}{r}130 \\
90\end{array}$ & $\begin{array}{l}\text { Rept. } \\
\text { Rept. }\end{array}$ & 360 & 162 & 16 & 150 & Elec. & 123.4 & Water-leve1 measurement on $3 / 7 / 79$ \\
\hline$S B-05-47-21-D B B 1$ & 402310102365701 & Corn & $\begin{array}{l}\text { Pivot } \\
\text { Flood }\end{array}$ & $\begin{array}{r}130 \\
70\end{array}$ & $\begin{array}{l}\text { Rept. } \\
\text { Rept. }\end{array}$ & 379 & 258 & 16 & 200 & Elec. & -- & Water-level measurement on $3 / 9 / 79$. \\
\hline$S B-05-48-35-B C A$ & 402140102420000 & Corn & Pivot & 130 & Rept. & 320 & 226 & 18 & 125 & Elec. & -- & Water-level measurement on $3 / 7 / 79$. \\
\hline SC-01-42-33-ACC & 395541102042400 & Alfalfa & Pivot & 140 & Rept. & -- & -- & -- & -- & -- & -- & \\
\hline SC-01-44-18-AAC & $395827 i 02205000$ & Corn & Flood & -- & $\cdots$ & 304 & -- & 16 & -- & N. gas & -- & $\begin{array}{l}\text { No acreage information in the well } \\
\text { folder. }\end{array}$ \\
\hline$S C-01-45-34-D B D$ & 395521102234800 & Corn & Pivoc & 180 & Rept. & 160 & -- & 22 & 100 & Elec. & - & \\
\hline$S C-01-46-31-D D B 1$ & 395523102334301 & Corn & Pivot & 120 & Rept. & -- & 103 & -- & 100 & Elec. & -- & Water-level measurement on $5 / 23 / 79$. \\
\hline SC-01-47-25-DDB & $395612 ? 02344800$ & Corn & Pivot & 130 & Rept. & $301^{\circ}$ & 109 & 18 & 100 & Elec. & -- & Water-level measurement on $5 / 23 / 79$. \\
\hline $\mathrm{SC}-01-48-08-\mathrm{BCAI}$ & 395906102464801 & Corn & Pivot & 160 & Rept. & 300 & 156 & - & - & Elec. & -- & Water-level measurement on $7 / 17 / 73$. \\
\hline
\end{tabular}


Table 8b.--Pumpage data for irrigation sites in Yuma County, Colorado--Continued

\begin{tabular}{|c|c|c|c|c|c|}
\hline \multirow{2}{*}{$\begin{array}{c}\text { Site } \\
\text { location }\end{array}$} & \multirow{2}{*}{$\begin{array}{c}\text { Field } \\
\text { person }\end{array}$} & \multicolumn{4}{|c|}{ Discharge information } \\
\hline & & Date & $\begin{array}{l}\text { Discharge } \\
\text { (gal/min) }\end{array}$ & Method & Remarks \\
\hline SB-05-46-34-DBC & JLP & $\begin{array}{l}8 / 06 / 79 \\
8 / 06 / 79 \\
8 / 06 / 79 \\
8 / 06 / 79\end{array}$ & $\begin{array}{r}850 \\
2,840 \\
1,050 \\
700\end{array}$ & $\begin{array}{l}\text { T.T. } \\
\text { Prop. } \\
\text { Dop. } \\
\text { T.T. }\end{array}$ & Measurement on 10 -in aluminum pipe. \\
\hline SB-05-47-21-DBB1 & JLP & $\begin{array}{l}8 / 03 / 79 \\
8 / 03 / 79\end{array}$ & $\begin{array}{l}1,400 \\
1,310\end{array}$ & $\begin{array}{l}\text { Inl. } \\
\text { Prop. }\end{array}$ & Measurement on $8-$ in aluminum pipe. \\
\hline$S B-05-48-35-B C A$ & JLP & $8 / 02 / 79$ & 615 & T.T. & Measurement on 8-in steel pipe. \\
\hline SC-01-42-33-ACC & JLP & -- & -- & -- & No discharge measurement made during 1979. \\
\hline SC-01-44-18-AAC & $\begin{array}{l}\text { CEP } \\
\text { RAP }\end{array}$ & $\begin{array}{l}8 / 02 / 79 \\
8 / 02 / 79\end{array}$ & $\begin{array}{l}715 \\
610\end{array}$ & $\begin{array}{l}\text { Dop. } \\
\text { Prop. }\end{array}$ & Measurement on 8-in aluminum-gated pipe. \\
\hline$S C-01-45-34-D B D$ & CEP & $7 / 29 / 79$ & 785 & T.T. & Measurement on 8-in steel tubing. \\
\hline SC-01-46-31-DDB1 & CEP & 9/06/79 & 260 & T.T. & $\begin{array}{l}\text { Field person felt this measurement was in error. } \\
\text { Measurement on 7-in steel pipe. }\end{array}$ \\
\hline$S C-01-47-25-D D B$ & CEP & $9 / 05 / 79$ & 780 & T.T. & Measurement on 8 -in steel tubing. \\
\hline $\mathrm{SC}-01-48-08-\mathrm{BCAI}$ & CEP & -- & -- & -- & No discharge measurement made during 1979 . \\
\hline
\end{tabular}


Table 8c.--Pumpage data for impigation sites in Yuma County, Colorado--Continued

Time of operation information

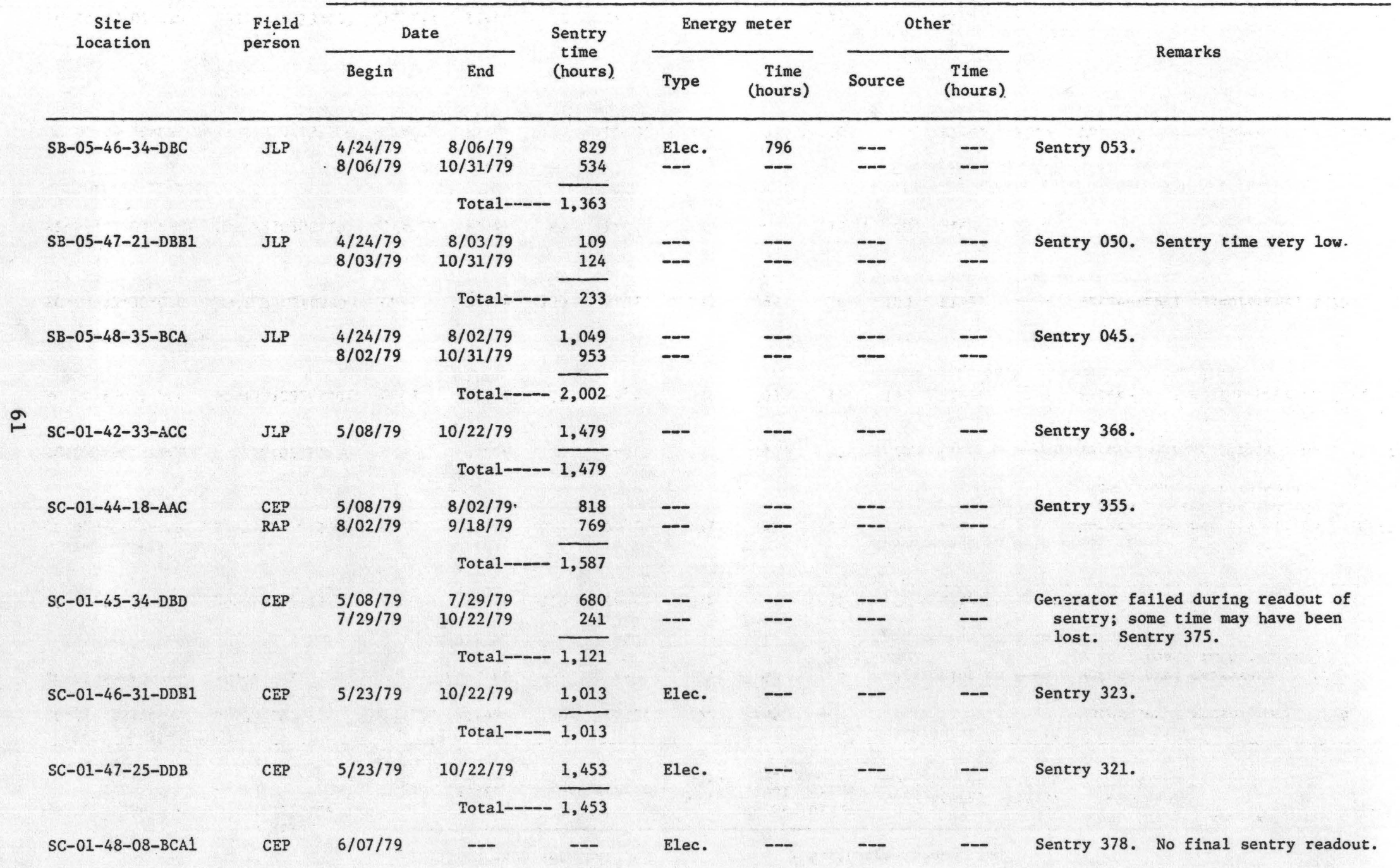


Table 8a.--Pumpage data for irrigation sites in Yuma County, Colorado--Continued

\begin{tabular}{|c|c|c|c|c|c|c|c|c|c|c|c|c|}
\hline \multirow[b]{2}{*}{$\begin{array}{c}\text { Site } \\
\text { location }\end{array}$} & \multirow[b]{2}{*}{$\begin{array}{c}\text { Station } \\
\text { identifier }\end{array}$} & \multicolumn{4}{|c|}{ Irrigated crop information } & \multicolumn{6}{|c|}{ Well information } & \multirow[b]{2}{*}{ Remarks } \\
\hline & & Type & System & Acres & Source & $\begin{array}{l}\text { Depth } \\
(\mathrm{ft})\end{array}$ & $\begin{array}{l}\text { Water } \\
\text { level } \\
\text { (ft) }\end{array}$ & $\begin{array}{l}\text { Diam- } \\
\text { meter } \\
(\text { in) }\end{array}$ & $\begin{array}{l}\text { Pump } \\
\text { hp. }\end{array}$ & $\begin{array}{l}\text { Energy } \\
\text { source }\end{array}$ & $\begin{array}{l}\text { Energy } \\
\text { rating }\end{array}$ & \\
\hline SC-01-48-18-DACl & 395753102471501 & Alfalfa & Pivot & 130 & Rept. & 270 & 165 & -- & $-\infty$ & Elec. & -- & Water-level measurement, 1976 \\
\hline$S C-02-45-10-B B B$ & 395416102243200 & Corn & Pivot & -- & $-\infty$ & 135 & 43 & 16 & 75 & Elec. & $-\infty$ & $\begin{array}{l}\text { Water-level measurement, } 8 / 57 \text {. } \\
\text { No acreage information. }\end{array}$ \\
\hline $\mathrm{SC}-02-46-20-\mathrm{AAC}$ & 395218102323500 & Corn & Pivot & 129 & Rept. & 365 & -- & 16 & 100 & Elec. & -- & \\
\hline $\mathrm{SC}-02-48-07-\mathrm{BDB}$ & 395355102475000 & Corn & Pivot & 130 & Rept. & 271 & 151 & 16 & 100 & Elec. & -- & $\begin{array}{l}\text { Water-level measurement on } 7 / 28 / 72 \\
\text { from observation well } 50 \mathrm{ft} \\
\text { southwest of this well. }\end{array}$ \\
\hline$S C-02-48-13-B A A$ & 395320102415000 & Alfalfa & Pivot & 170 & Rept. & 227 & 160 & 16 & 75 & Elec. & -- & Water-level measurement on $11 / 21 / 71$. \\
\hline $\mathrm{SC}-04-45-21-\mathrm{ACD}$ & 394138102243801 & Corn & Flood & 90 & Rept. & 339 & 203 & 16 & 150 & Elec. & 123.4 & Water-level measurement on $5 / 22 / 73$.. \\
\hline SC-04-47-30-CBD & 394027102405900 & Corn & Pivot & 130 & Rept. & 245 & 95 & 16 & 100 & Elec. & -- & Water-level measurement, $1 / 70$. \\
\hline $\mathrm{SC}-05-42-05-\mathrm{ADB} 1$ & 393922102051101 & Alfalfa & Pivot & 85 & Rept. & 80 & -- & 18 & 60 & Elec. & 38.8 & \\
\hline SC-05-43-18-CBB & 393720102135500 & Alfalfa & Pivot & 112 & Meas. & 112 & 37 & 16 & -- & Elec. & 32.9 & Water-level measurement on $5 / 15 / 56$. \\
\hline$S C-05-43-07-C B D$ & 393810102135000 & Alfalfa & Flood & 90 & Rept. & 240 & $-\infty$ & 16 & 50 & Elec. & --- & \\
\hline
\end{tabular}


Table 8b.--Pumpage data for irrigation sites in Yuma County, Colorado--Continued

\begin{tabular}{|c|c|c|c|c|c|}
\hline \multirow{2}{*}{$\begin{array}{c}\text { Site } \\
\text { location }\end{array}$} & \multirow{2}{*}{$\begin{array}{r}\text { Field } \\
\text { person }\end{array}$} & \multicolumn{4}{|c|}{ Discharge information } \\
\hline & & Date & $\begin{array}{l}\text { Discharge } \\
\text { (gal/min) }\end{array}$ & Method & Remarks \\
\hline SC-01-48-18-DAC1 & CEP & -- & $\cdots$ & -- & No discharge measurement made during 1979. \\
\hline$S C-02-45-10-8 B B$ & CEP & -- & -- & -- & No discharge measurement made during 1979. \\
\hline $\mathrm{SC}-02-46-20-\mathrm{AAC}$ & CEP & -- & -- & -- & No discharge measurement made during 1979. \\
\hline$S C-02-48-07-B D B$ & CEP & -- & -- & -- & No discharge measurement made during 1979. \\
\hline $\mathrm{SC}-02-48-13-\mathrm{BAA}$ & CEP & $9 / 05 / 79$ & 510 & T.T. & Measurement on 8 -in steel tubing. \\
\hline $\mathrm{SC}-04-45-21-\mathrm{ACD}$ & $\begin{array}{l}\text { FJH } \\
\text { RGB }\end{array}$ & $\begin{array}{l}8 / 17 / 79 \\
8 / 17 / 79\end{array}$ & $\begin{array}{l}1,410 \\
1,390\end{array}$ & $\begin{array}{l}\text { T.T. } \\
\text { Dop. }\end{array}$ & $\begin{array}{l}\text { Measurements on 10-in aluminum-gated pipe; spacing } 9 \text { on } \\
\text { clampitron. }\end{array}$ \\
\hline$S C-04-47-30-C B D$ & CEP & $9 / 06 / 79$ & -- & T.T. & $\begin{array}{l}\text { Could not get averaged reading on clampitron for } \\
7 \text {-in steel pipe. }\end{array}$ \\
\hline$S C-05-42-05-A D B 1$ & $\begin{array}{l}\text { F JH } \\
\text { CEP }\end{array}$ & $\begin{array}{l}8 / 19 / 79 \\
8 / 19 / 79\end{array}$ & $\begin{array}{l}280 \\
215\end{array}$ & $\begin{array}{l}\text { T.T. } \\
\text { Dop. }\end{array}$ & Measurement on 8-in steel pipe. \\
\hline$S C-05-43-18-C B B$ & $\begin{array}{l}\text { CEP } \\
\text { FJH }\end{array}$ & $\begin{array}{l}8 / 19 / 79 \\
8 / 19 / 79 \\
8 / 19 / 79\end{array}$ & $\begin{array}{l}310 \\
330 \\
375\end{array}$ & $\begin{array}{l}\text { T.T. } \\
\text { Dop. } \\
\text { Inl. }\end{array}$ & $\begin{array}{l}\text { Measurement on } 6 \text {-in steel pipe. } \\
\text { Measurement on } 8 \text {-in steel tubing. }\end{array}$ \\
\hline $\mathrm{SC}-05-43-07-\mathrm{CBD}$ & CEP & $9 / 07 / 79$ & 225 & T.T. & Measurement on 6-in aluminum-gated pipe. \\
\hline
\end{tabular}


Table 8c.--Pumpage data for irrigation sites in Yuma County, Colorado--Continued

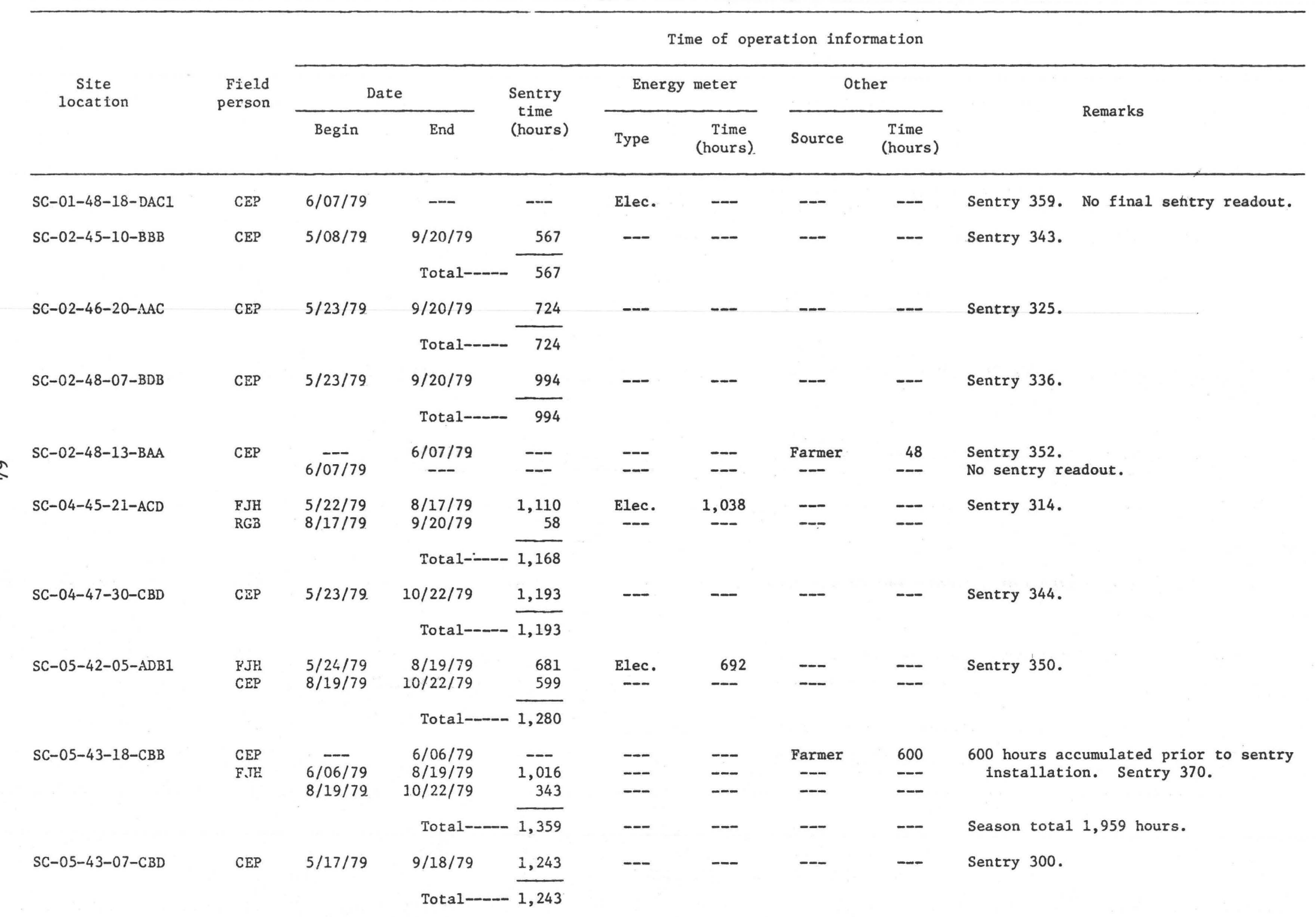


Table 8a.--Pumpage data for irrigation sites in Yuma County, Colorado--Continued

\begin{tabular}{|c|c|c|c|c|c|c|c|c|c|c|c|c|}
\hline \multirow[b]{2}{*}{$\begin{array}{l}\text { Site } \\
\text { location }\end{array}$} & \multirow{2}{*}{$\begin{array}{l}\text { Station } \\
\text { idencifler }\end{array}$} & \multicolumn{4}{|c|}{ Irrigated crop information } & \multicolumn{6}{|c|}{ Well information } & \multirow[b]{2}{*}{ Remarks } \\
\hline & & Type & System & Acres & Source & $\begin{array}{l}\text { Depth } \\
(\mathrm{ft})\end{array}$ & $\begin{array}{r}\text { Water } \\
\text { level } \\
\text { (ft) }\end{array}$ & $\begin{array}{l}\text { Diam- } \\
\text { meter } \\
\text { (in) }\end{array}$ & $\begin{array}{l}\text { Pump } \\
\text { hp. }\end{array}$ & $\begin{array}{l}\text { Energy } \\
\text { source }\end{array}$ & $\begin{array}{l}\text { Energy } \\
\text { rating }\end{array}$ & \\
\hline SC-05-46-35-CBB & 393440102294300 & Corn & Pivot & 160 & Rept. & 206 & 114 & -- & 100 & Elec. & -- & Water-level measurement on $5 / 17 / 79$. \\
\hline$S C-05-48-04-C D B$ & 393840102450000 & Corn & Pivot & 120 & Rept. & 234 & 129 & -- & 125 & Elec. & -- & Water-level measurement on $5 / 18 / 79$. \\
\hline
\end{tabular}


Table 8b.--Pumpage data for irrigation sites in Yuma County, Colorado--Continued

\begin{tabular}{|c|c|c|c|c|c|}
\hline \multirow{2}{*}{$\begin{array}{c}\text { Site } \\
\text { location }\end{array}$} & \multirow{2}{*}{$\begin{array}{r}\text { Field } \\
\text { person }\end{array}$} & \multicolumn{4}{|c|}{ Discharge information } \\
\hline & & Date & $\begin{array}{l}\text { Discharge } \\
\text { (gal/min) }\end{array}$ & Method & Remarks \\
\hline
\end{tabular}

SC-05-46-35-CBB

CEP

CEP

$-\cdots$

$--$ $-\cdots$

$\cdots$
$--$

$--$
No discharge measurement made during 1979 .

No discharge measurement made during 1979. 
Table 8c.--Pumpage data for irrigation sites in Yuma County, Colorado--Continued

\begin{tabular}{|c|c|c|c|c|c|c|c|c|c|c|}
\hline \multirow{3}{*}{$\begin{array}{c}\text { Site } \\
\text { location }\end{array}$} & \multirow{3}{*}{$\begin{array}{r}\text { Field } \\
\text { person }\end{array}$} & \multicolumn{9}{|c|}{ Time of operation information } \\
\hline & & \multicolumn{2}{|c|}{ Date } & \multirow{2}{*}{$\begin{array}{l}\text { Sentry } \\
\text { time } \\
\text { (hours) }\end{array}$} & \multicolumn{2}{|c|}{ Energy meter } & \multicolumn{2}{|c|}{ Other } & \multirow{2}{*}{\multicolumn{2}{|c|}{ Remarks }} \\
\hline & & Begin & End & & Type & $\begin{array}{c}\text { Time } \\
\text { (hours) }\end{array}$ & Source & $\begin{array}{l}\text { Time } \\
\text { (hours) }\end{array}$ & & \\
\hline \multirow[t]{2}{*}{ SC $-05-46-35-С B B$} & CEP & $5 / 17 / 79$ & $9 / 18 / 79$ & 1,242 & -- & -- & $-\infty$ & -- & Sentry 305. & \\
\hline & & & Total- & 1,242 & & & & & & \\
\hline \multirow[t]{2}{*}{$S C-05-48-04-C D B$} & CEP & $5 / 18 / 79$ & $9 / 19 / 79$ & 1,174 & -- & -- & -- & $-\infty$ & Sentry 311. & \\
\hline & & & Total- & 1,174 & & & & & & \\
\hline
\end{tabular}


Table 9a.--Pumpage data for irrigation sites in Cheyenne County, Kansas

\begin{tabular}{|c|c|c|c|c|c|c|c|c|c|c|c|c|}
\hline \multirow{2}{*}{$\begin{array}{c}\text { Site } \\
\text { location }\end{array}$} & \multirow{2}{*}{$\begin{array}{l}\text { Station } \\
\text { identifier }\end{array}$} & \multicolumn{4}{|c|}{ Irrigated crop information } & \multicolumn{6}{|c|}{ Well information } & \multirow[b]{2}{*}{ Remarks } \\
\hline & & Type & System A & Acres & Source & $\begin{array}{r}\text { Depth } \\
(\mathrm{ft})\end{array}$ & $\begin{array}{r}\text { Water } \\
\text { level } \\
(\mathrm{ft})\end{array}$ & $\begin{array}{l}\text { Diam- } \\
\text { meter } \\
\text { (in) }\end{array}$ & $\begin{array}{l}\text { Pump } \\
\text { hp. }\end{array}$ & $\begin{array}{l}\text { Energy } \\
\text { source }\end{array}$ & $\begin{array}{l}\text { Energy } \\
\text { rating }\end{array}$ & \\
\hline SC-01-38-01-BBC & $-\cdots$ & Alfalfa & $\begin{array}{l}\text { Sprink- } \\
\text { ler }\end{array}$ & 70 & Rept. & -- & -- & -- & 25 & Elec. & 15.4 & \\
\hline SC-02-40-25-ADA & 395112101450302 & Corn & Flocd & 55 & Meas. & -- & -- & $-\cdots$ & 30 & Elec. & --- & \\
\hline SC-02-41-27-BBD & $-\cdots$ & $\begin{array}{l}\text { Alfalfa } \\
\text { Corn }\end{array}$ & Flood & $\begin{array}{l}10 \\
58\end{array}$ & $\begin{array}{l}\text { Rept. } \\
\text { Rept. }\end{array}$ & -- & -- & -- & 60 & Elec. & 28 & \\
\hline SC-03-37-24-ACC & $-\cdots$ & Corn & Pivot & 130 & Meas. & -- & -- & -- & -- & N. gas & -- & \\
\hline$S C-03-39-24-D A B$ & 394639101383401 & Corn & Flood & 33 & Rept. & -- & -- & $-\infty$ & -- & N. gas & -- & \\
\hline SC-03-40-29-ABC & 394607101500101 & Alfalfa & $\begin{array}{l}\text { Sprink- } \\
\quad \text { ler }\end{array}$ & 26 & Rept. & - & -- & -- & -- & Elec. & -- & \\
\hline $\mathrm{SC}-03-42-33-\mathrm{CCB}$ & 394442102024701 & $\begin{array}{l}\text { Corn } \\
\text { Alfalfa }\end{array}$ & $\begin{array}{l}\text { Sprink- } \\
\text { ler }\end{array}$ & $\begin{array}{l}32 \\
50\end{array}$ & $\begin{array}{l}\text { Rept. } \\
\text { Rept. }\end{array}$ & -- & -- & -- & -- & N. gas & -- & \\
\hline$S C-04-37-23-C C B$ & $\ldots$ & $\begin{array}{l}\text { Corn } \\
\text { Alfalfa }\end{array}$ & Flood & $\begin{array}{l}48 \\
12\end{array}$ & Meas. & -- & -- & -- & -- & N. gas & -- & \\
\hline $\mathrm{SC}-04-38-21-\mathrm{ADC}$ & $-\cdots$ & Corn & Flood & 108 & Meas. & -- & -- & -- & -- & N. gas & -- & \\
\hline
\end{tabular}


Table 9b.--Pumpage data for irrigation sites in Cheyenne County, Kansas

\begin{tabular}{|c|c|c|c|c|c|}
\hline \multirow{2}{*}{$\begin{array}{l}\text { Site } \\
\text { location }\end{array}$} & \multirow{2}{*}{$\begin{array}{r}\text { Field } \\
\text { person }\end{array}$} & \multicolumn{4}{|c|}{ Discharge information } \\
\hline & & Date & $\begin{array}{l}\text { Discharge } \\
\text { (gal/min) }\end{array}$ & Method & Remarks \\
\hline $\mathrm{SC}-01-38-01-\mathrm{BBC}$ & JLP & $\begin{array}{l}7 / 17 / 79 \\
7 / 17 / 79\end{array}$ & $\begin{array}{l}565 \\
405\end{array}$ & $\begin{array}{l}\text { Dop. } \\
\text { Dop. }\end{array}$ & $\begin{array}{l}\text { Measurement on } 6 \text {-in aluminum pipe. } \\
\text { Polysonics meter. }\end{array}$ \\
\hline $\mathrm{SC}-02-40-25-\mathrm{ADA}$ & $\begin{array}{l}\text { FJH } \\
\text { RGB }\end{array}$ & $\begin{array}{l}8 / 18 / 79 \\
8 / 18 / 79\end{array}$ & $\begin{array}{l}340 \\
340\end{array}$ & $\begin{array}{l}\text { T.T. } \\
\text { Dop. }\end{array}$ & $\begin{array}{l}\text { Measurement on 6-in steel pipe. } \\
\text { Measurement on 6-in aluminum-gated pipe. }\end{array}$ \\
\hline SC-02-41-27-SBD & $\begin{array}{l}\text { FJH } \\
\text { RGB }\end{array}$ & $\begin{array}{l}8 / 18 / 79 \\
8 / 18 / 79 \\
8 / 18 / 79\end{array}$ & $\begin{array}{l}305 \\
200 \\
300\end{array}$ & $\begin{array}{l}\text { T.T. } \\
\text { Dop. } \\
\text { Prop. }\end{array}$ & $\begin{array}{l}\text { Measurement on 6-in steel pipe. } \\
\text { Measurement on } 6 \text {-in aluminum-gated pipe. }\end{array}$ \\
\hline SC-03-37-24-ACC & $\begin{array}{l}\text { FJH } \\
\text { RGB }\end{array}$ & $\begin{array}{l}8 / 18 / 79 \\
8 / 18 / 79\end{array}$ & $\begin{array}{l}355 \\
280\end{array}$ & $\begin{array}{l}\text { T.T. } \\
\text { Dop. }\end{array}$ & Measurement on 8 -in steel tubing. \\
\hline $\mathrm{SC}-03-39-24-\mathrm{DAB}$ & BDR & -- & -- & $\cdots$ & No discharge measurement. \\
\hline $\mathrm{SC}-03-40-29-\mathrm{ABC}$ & BDR & $9 / 11 / 79$ & 110 & Dop. & Measurement on $4-$ in aluminum pipe. \\
\hline $\mathrm{SC}-03-42-33-\mathrm{CCB}$ & BDR & $\begin{array}{l}9 / 11 / 79 \\
9 / 11 / 79\end{array}$ & $\begin{array}{l}155 \\
195\end{array}$ & $\begin{array}{l}\text { T.T. } \\
\text { Dop. }\end{array}$ & Measurement on 6-in steel pipe. \\
\hline $\mathrm{SC}-04-37-23-\mathrm{CCB}$ & BDR & $\begin{array}{l}8 / 26 / 79 \\
8 / 26 / 79\end{array}$ & $\begin{array}{l}405 \\
345\end{array}$ & $\begin{array}{l}\text { T.T. } \\
\text { Prop. }\end{array}$ & Measurement on 8-in aluminum-gated pipe. \\
\hline$S C-04-38-21-A D C$ & BDR & - & -- & -- & No discharge measurement made during 1979 \\
\hline
\end{tabular}


Table 9c.--Pumpage data for irrigation sites in Cheyenne County, Kansas

Time of operation information

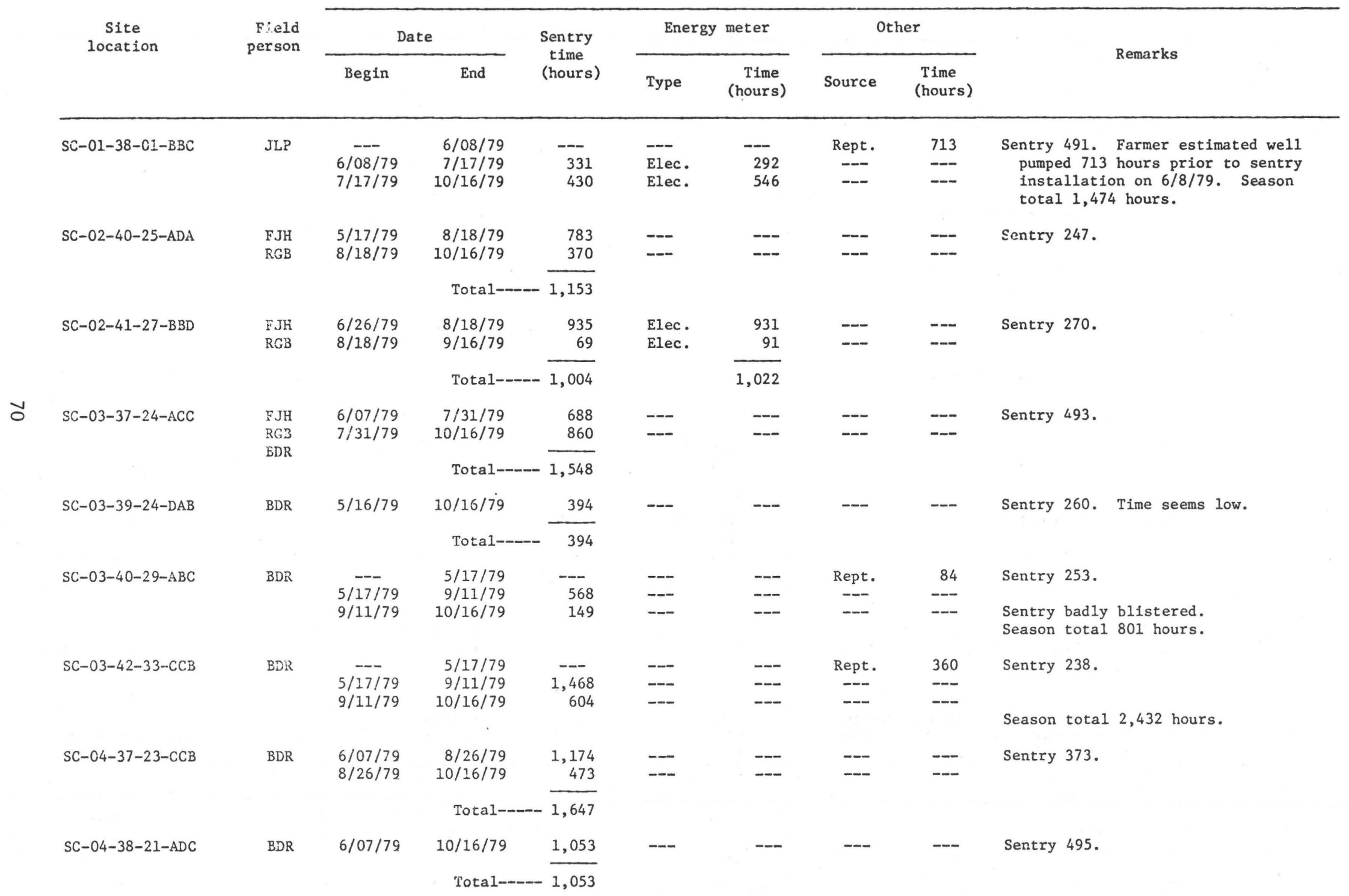


Table 9a.--Pumpage data for irrigation sites in Cheyenne County, Kansas--Continued

\begin{tabular}{|c|c|c|c|c|c|c|c|c|c|c|c|c|}
\hline \multirow{2}{*}{$\begin{array}{c}\text { Site } \\
\text { location }\end{array}$} & \multirow{2}{*}{$\begin{array}{l}\text { Station } \\
\text { identifier }\end{array}$} & \multicolumn{4}{|c|}{ Irrigated crop information } & \multicolumn{6}{|c|}{ Well information } & \multirow[b]{2}{*}{ Remarks } \\
\hline & & Type & System & Acres & Source & $\begin{array}{r}\text { Depth } \\
\text { (ft) }\end{array}$ & $\begin{array}{r}\text { Water } \\
\text { level } \\
(\mathrm{ft})\end{array}$ & $\begin{array}{l}\text { Diam- } \\
\text { meter } \\
\text { (in) }\end{array}$ & $\begin{array}{l}\text { Pump } \\
\text { hp. }\end{array}$ & $\begin{array}{l}\text { Energy } \\
\text { source }\end{array}$ & $\begin{array}{l}\text { Energy } \\
\text { rating }\end{array}$ & \\
\hline SC-05-38-20-DAA & $393612 \mathrm{i} 01361601$ & Corn & Flood & 25 & Rept. & $-\cdots$ & - & -- & 50 & Elec. & -- & \\
\hline sc-05-41-22-cc & $\cdots$ & Corn & Flood & 160 & Rept. & -- & -- & -- & -- & N. gas & $\ldots$ & \\
\hline
\end{tabular}


Table 9b.--Pumpage data for irrigation sites in Cheyenne County, Kansas--Continued

\begin{tabular}{|c|c|c|c|c|c|}
\hline \multirow{2}{*}{$\begin{array}{c}\text { Site } \\
\text { location }\end{array}$} & \multirow{2}{*}{$\begin{array}{r}\text { Field } \\
\text { person }\end{array}$} & \multicolumn{4}{|c|}{ Discharge information } \\
\hline & & Date & $\begin{array}{l}\text { Discharge } \\
\text { (gal/min) }\end{array}$ & Method & Remarks \\
\hline SC-05-38-20-DAA & BDR & -- & 600 & Rept. & Owner estimate. \\
\hline SC-05-41-22-CC & $\begin{array}{l}\mathrm{BDR} \\
\mathrm{CH}\end{array}$ & $\begin{array}{l}6 / 20 / 79 \\
6 / 20 / 79\end{array}$ & $\begin{array}{l}810 \\
675\end{array}$ & $\begin{array}{l}\text { T.T. } \\
\text { Dop. }\end{array}$ & Measurement on 8-in aluminum pipe. \\
\hline
\end{tabular}


Table 9c.--Pumpage data for irrigation sites in Cheyenne County, Kansas--Continued

\begin{tabular}{|c|c|c|c|c|c|c|c|c|c|c|}
\hline \multirow{3}{*}{$\begin{array}{c}\text { Site } \\
\text { location }\end{array}$} & \multirow{3}{*}{$\begin{array}{r}\text { Field } \\
\text { person }\end{array}$} & \multicolumn{9}{|c|}{ Time of operation information } \\
\hline & & \multicolumn{2}{|c|}{ Date } & \multirow{2}{*}{$\begin{array}{l}\text { Sentry } \\
\text { time } \\
\text { (hours) }\end{array}$} & \multicolumn{2}{|c|}{ Energy meter } & \multicolumn{2}{|c|}{ Other } & \multirow{2}{*}{\multicolumn{2}{|c|}{ Remarks }} \\
\hline & & Begin & End & & Type & $\begin{array}{c}\text { Time } \\
\text { (hours) }\end{array}$ & Source & $\begin{array}{l}\text { Time } \\
\text { (hours) }\end{array}$ & & \\
\hline \multirow[t]{2}{*}{$S C-05-38-20-D A A$} & $\mathrm{BDR}$ & $5 / 16 / 79$ & $10 / 16 / 79$ & 668 & -- & -- & -- & -- & Sentry & 258. \\
\hline & & & Total-- & 668 & & & & & & \\
\hline \multirow[t]{2}{*}{ SC $-05-41-22-C C$} & $\begin{array}{l}\mathrm{BDR} \\
\mathrm{CH}\end{array}$ & $\begin{array}{l}5 / 16 / 79 \\
6 / 20 / 79\end{array}$ & $\begin{array}{r}6 / 20 / 79 \\
10 / 17 / 79\end{array}$ & $\begin{array}{r}605 \\
1,372\end{array}$ & -- & -- & -- & -- & Sentry & 239. \\
\hline & & & Total-- & $-1,977$ & & & & & & \\
\hline
\end{tabular}


Table 10a.--Pumpage data for irrigation sites in Sherman County, Kansas

\begin{tabular}{|c|c|c|c|c|c|c|c|c|c|c|c|c|}
\hline \multirow{2}{*}{$\begin{array}{c}\text { Site } \\
\text { location }\end{array}$} & \multirow{2}{*}{$\begin{array}{l}\text { Station } \\
\text { identifier }\end{array}$} & \multicolumn{4}{|c|}{ Irrigated crop information } & \multicolumn{6}{|c|}{ Well informatior. } & \multirow[b]{2}{*}{ Remarks } \\
\hline & & Type & System & Acres & Source & $\begin{array}{c}\text { Depth } \\
(\mathrm{ft})\end{array}$ & $\begin{array}{r}\text { Water } \\
\text { level } \\
\text { (ft) }\end{array}$ & $\begin{array}{l}\text { Diam- } \\
\text { meter } \\
\text { (in) }\end{array}$ & $\begin{array}{l}\text { Pump } \\
\text { hp. }\end{array}$ & $\begin{array}{l}\text { Energy } \\
\text { source }\end{array}$ & $\begin{array}{l}\text { Energy } \\
\text { rating }\end{array}$ & \\
\hline SC-06-37-02-CAC & ---- & Corn & Pivot & 125 & Rept. & -- & -- & --- & --- & N. gas & -- & \\
\hline$S C-06-37-14-D B C$ & ---- & Corn & Pivot & 100 & Rept. & -- & -- & --- & -- & N. gas & -- & \\
\hline$S C-06-37-16-C D D$ & $-\cdots$ & $\begin{array}{r}\text { Corn } \\
\text { Grass }\end{array}$ & Pivot & $\begin{array}{l}46 \\
47\end{array}$ & $\begin{array}{l}\text { Meas. } \\
\text { Meas. }\end{array}$ & -- & -- & -- & -- & N. gas & -- & \\
\hline $\mathrm{SC}-06-37-17-\mathrm{ACD}$ & --- & Corn & Pivot & 120 & Rept. & -- & -- & --- & -- & N. gas & --- & \\
\hline$S C-06-40-10-D B C$ & ---- & Corn & Pivot & 130 & Rept. & -- & -- & --- & 100 & Elec. & 71.5 & \\
\hline SC-06-41-23-C & 393040101521801 & Corn & Pivot & 250 & Rept. & -- & -- & -- & 200 & Elec. & --- & \\
\hline SC-C6-41-27-DBD & ---- & Corn & Flood & 130 & Rept. & -- & 161.5 & -- & -- & N. gas & $-\cdots$ & Water-1eve1 measurement on $1 / 3 / 79$. \\
\hline SC-07-37-10-BCA & $-\cdots$ & Corn & Pivot & 260 & Rept. & -- & -- & --- & --- & N. gas & --- & \\
\hline $\mathrm{SC}-07-39-17-\mathrm{ACC}$ & ----- & Corn & Flood & 88 & Rept. & --- & -- & --- & -- & N. gas & -- & \\
\hline
\end{tabular}


Table 10b.--Pumpage data for irrigation sites in Sherman County, Kansas

\begin{tabular}{|c|c|c|c|c|c|}
\hline \multirow{2}{*}{$\begin{array}{l}\text { Site } \\
\text { location }\end{array}$} & \multirow{2}{*}{$\begin{array}{r}\text { Field } \\
\text { person }\end{array}$} & \multicolumn{4}{|c|}{ Discharge information } \\
\hline & & Date & $\begin{array}{l}\text { Discharge } \\
\text { (gal/min) }\end{array}$ & Method & Remarks \\
\hline SC-06-37-02-CAC & $\mathrm{BDR}$ & $\begin{array}{l}7 / 31 / 79 \\
7 / 31 / 79\end{array}$ & $\begin{array}{l}450 \\
220\end{array}$ & $\begin{array}{l}\text { T.T. } \\
\text { Dop. }\end{array}$ & Measurement on 8-in aluminum pipe. \\
\hline$S C-06-37-14-D B C$ & JLP & $7 / 09 / 79$ & 860 & Dop. & Measurement on 8-in aluminum pipe. \\
\hline$S C-06-37-16-C D D$ & JLP & $7 / 09 / 79$ & 425 & Dop. & Measurement on 6-in steel pipe. \\
\hline SC-06-37-17-ACD & JLP & $\begin{array}{l}7 / 09 / 79 \\
7 / 09 / 79\end{array}$ & $\begin{array}{l}1,350 \\
1,020\end{array}$ & $\begin{array}{l}\text { Dop. } \\
\text { Dop. }\end{array}$ & $\begin{array}{l}\text { Measurement on } 8 \text {-in steel tubing. } \\
\text { Polysonics meter. }\end{array}$ \\
\hline$S C-06-40-10-D B C$ & JLP & $7 / 09 / 79$ & 855 & Dop. & Measurement on 6-in steel pipe. \\
\hline SC-06-41-23-C & JLP & $\begin{array}{l}7 / 13 / 79 \\
7 / 13 / 79\end{array}$ & $\begin{array}{l}1,160 \\
1,635\end{array}$ & $\begin{array}{l}\text { T.T. } \\
\text { Dop. }\end{array}$ & Measurement on 8-in steel pipe. \\
\hline SC-06-41-27-DBD & JLP & $\begin{array}{l}7 / 06 / 79 \\
7 / 06 / 79\end{array}$ & $\begin{array}{r}840 \\
1,195\end{array}$ & $\begin{array}{l}\text { Dop. } \\
\text { Prop. }\end{array}$ & Measurement on 9-in aluminum-gated pipe. \\
\hline SC $-07-37-10-B C A$ & $\begin{array}{l}\mathrm{JLP} \\
\mathrm{KRW}\end{array}$ & $\begin{array}{l}7 / 19 / 79 \\
7 / 19 / 79\end{array}$ & $\begin{array}{l}595 \\
175\end{array}$ & $\begin{array}{l}\text { T.T. } \\
\text { Dop. }\end{array}$ & Measurement on 8-in steel pipe. \\
\hline $\mathrm{SC}-07-39-17-\mathrm{ACC}$ & BDR & $8 / 26 / 79$ & 350 & T.T. & Measurement on 8-in aluminum-gated pipe. \\
\hline
\end{tabular}


Table 10c.--Pumpage data for irrigation sites in Sherman County, Kansas

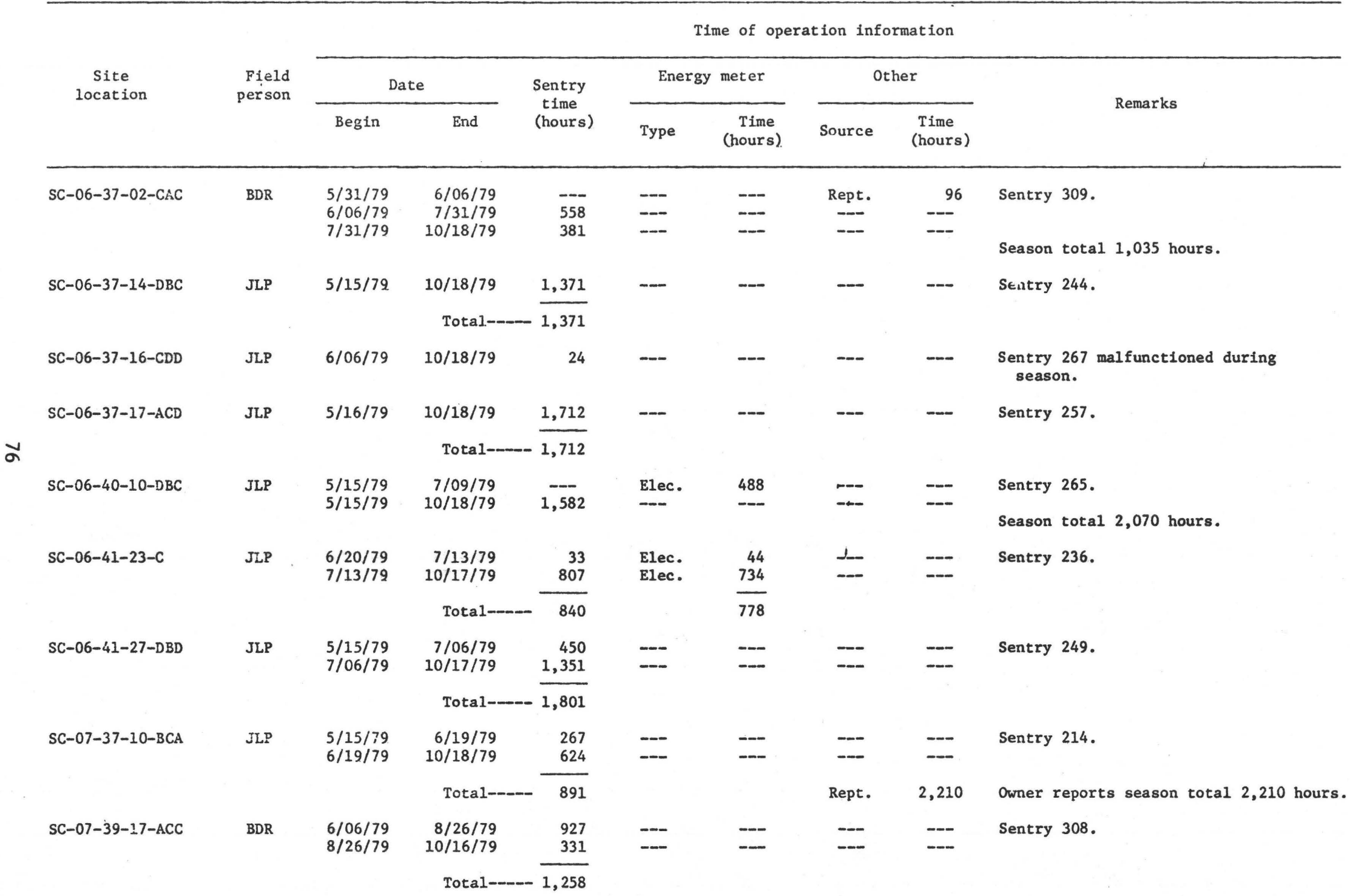


Table 10a.--Pumpage data for irrigation sites in Sherman County, Kansas--Continued

\begin{tabular}{|c|c|c|c|c|c|c|c|c|c|c|c|c|}
\hline \multirow{2}{*}{$\begin{array}{c}\text { Site } \\
\text { location }\end{array}$} & \multirow{2}{*}{$\begin{array}{l}\text { Station } \\
\text { identifier }\end{array}$} & \multicolumn{4}{|c|}{ Irrigated crop information } & \multicolumn{6}{|c|}{ Well information } & \multirow{2}{*}{ Remarks } \\
\hline & & Type & System & Acres & Source & $\begin{array}{c}\text { Depth } \\
(\mathrm{ft})\end{array}$ & $\begin{array}{r}\text { Water } \\
\text { level } \\
(\mathrm{ft})\end{array}$ & $\begin{array}{l}\text { Diam- } \\
\text { meter } \\
\text { (in) }\end{array}$ & $\begin{array}{l}\text { Pump } \\
\text { hp. }\end{array}$ & $\begin{array}{l}\text { Energy } \\
\text { source }\end{array}$ & $\begin{array}{l}\text { Energy } \\
\text { rating }\end{array}$ & \\
\hline SC-07-41-01-ABD & $-\cdots$ & $\begin{array}{l}\text { Beets } \\
\text { Beans }\end{array}$ & Flood & $\begin{array}{l}55 \\
70\end{array}$ & Rept. & -- & -- & -- & -- & N. gas & -- & \\
\hline SC-07-41-32-CCD & 392342101553501 & Alfalfa & Pivot & 120 & Rept. & -- & -- & -- & -- & N. gas & 0.16 & \\
\hline$S C-07-41-33-A B A$ & $\cdots$ & Corn & Pivot & 120 & Rept. & -- & -- & -- & -- & N. gas & - & \\
\hline$S C-08-37-04-B B B$ & $-\cdots$ & Corn & Flood & 155 & Rept. & $-\infty$ & -- & -- & $-\cdots$ & N. gas & -- & \\
\hline$S C-08-37-28-B B D$ & $\ldots$ & Corn & Pivot & 120 & Rept. & - & -- & - & -- & N. gas & - & \\
\hline $\mathrm{SC}-08-38-01-\mathrm{BDA}$ & $\ldots$ & Corn & Fiood & 150 & Rept. & -- & -- & - & -- & N. gas & $\cdots$ & \\
\hline $\mathrm{SC}-08-38-16-\mathrm{BCD}$ & $\ldots$ & $\begin{array}{l}\text { Corn } \\
\text { Milo }\end{array}$ & Flood & $\begin{array}{r}60 \\
130\end{array}$ & Rept. & -- & $-\cdots$ & -- & -- & N. gas & -- & \\
\hline $\mathrm{SC}-08-38-32-\mathrm{BAA}$ & $-\cdots$ & Corn & Flood & 140 & Rept. & -- & -- & - & - & N. gas & .32 & \\
\hline
\end{tabular}


Table 10b.--Pumpage data for irrigation sites in Sherman County, Kansas--Continued

\begin{tabular}{|c|c|c|c|c|c|}
\hline \multirow{2}{*}{$\begin{array}{l}\text { Site } \\
\text { location }\end{array}$} & \multirow{2}{*}{$\begin{array}{r}\text { Field } \\
\text { person }\end{array}$} & \multicolumn{4}{|c|}{ Discharge information } \\
\hline & & Date & $\begin{array}{l}\text { Discharge } \\
\text { (gal/min) }\end{array}$ & Method & Remarks \\
\hline $\mathrm{SC}-07-41-01-\mathrm{ABD}$ & $\mathrm{BDR}$ & $9 / 11 / 79$ & 760 & T.T. & Measurement on 8 -in steel pipe. \\
\hline$S C-07-41-32-C C D$ & $\mathrm{BDR}$ & $8 / 26 / 79$ & 465 & T.T. & Measurement on 8-in steel tubing. \\
\hline$S C-07-41-33-A B A$ & BDR & $\begin{array}{l}7 / 11 / 79 \\
8 / 26 / 79\end{array}$ & $\begin{array}{l}735 \\
565\end{array}$ & $\begin{array}{l}\text { Dop. } \\
\text { T.T. }\end{array}$ & Measurement on 6-in steel tubing. \\
\hline$S C-08-37-04-B B B$ & BDR & $\begin{array}{l}7 / 30 / 79 \\
7 / 30 / 79\end{array}$ & $\begin{array}{r}810 \\
1,000\end{array}$ & $\begin{array}{l}\text { Dop. } \\
\text { Rept. }\end{array}$ & $\begin{array}{l}\text { Measurement in } 10-\text { in aluminum-gated pipe. } \\
\text { Owner estimate. }\end{array}$ \\
\hline SC-08-37-28-BBD & BDR & $\begin{array}{l}8 / 09 / 79 \\
8 / 09 / 79\end{array}$ & $\begin{array}{l}670 \\
235\end{array}$ & $\begin{array}{l}\text { T.T. } \\
\text { Dop. }\end{array}$ & Measurement on 8 -in steel tubing. \\
\hline$S C-08-38-01-B D A$ & JLP & -- & -- & -- & No discharge information. \\
\hline $\mathrm{SC}-08-38-16-\mathrm{BCD}$ & $\mathrm{BDR}$ & $\begin{array}{l}8 / 09 / 79 \\
8 / 09 / 79\end{array}$ & $\begin{array}{r}980 \\
1,080\end{array}$ & $\begin{array}{l}\text { T.T. } \\
\text { Dop. }\end{array}$ & Measurement on 8-in aluminum-gated pipe. \\
\hline SC-08-38-32-BAA & $\begin{array}{l}\text { FJH } \\
\text { RGB } \\
\text { BDR }\end{array}$ & $\begin{array}{l}8 / 17 / 79 \\
8 / 17 / 79 \\
8 / 17 / 79\end{array}$ & $\begin{array}{r}1,240 \\
915 \\
775\end{array}$ & $\begin{array}{l}\text { T.T. } \\
\text { Dop. } \\
\text { Prop. }\end{array}$ & Measurement on 10-in aluminum-gated pipe. \\
\hline
\end{tabular}


Table 10c.--Pumpage data for irrigation sites in Sherman County, Kansas--Continued

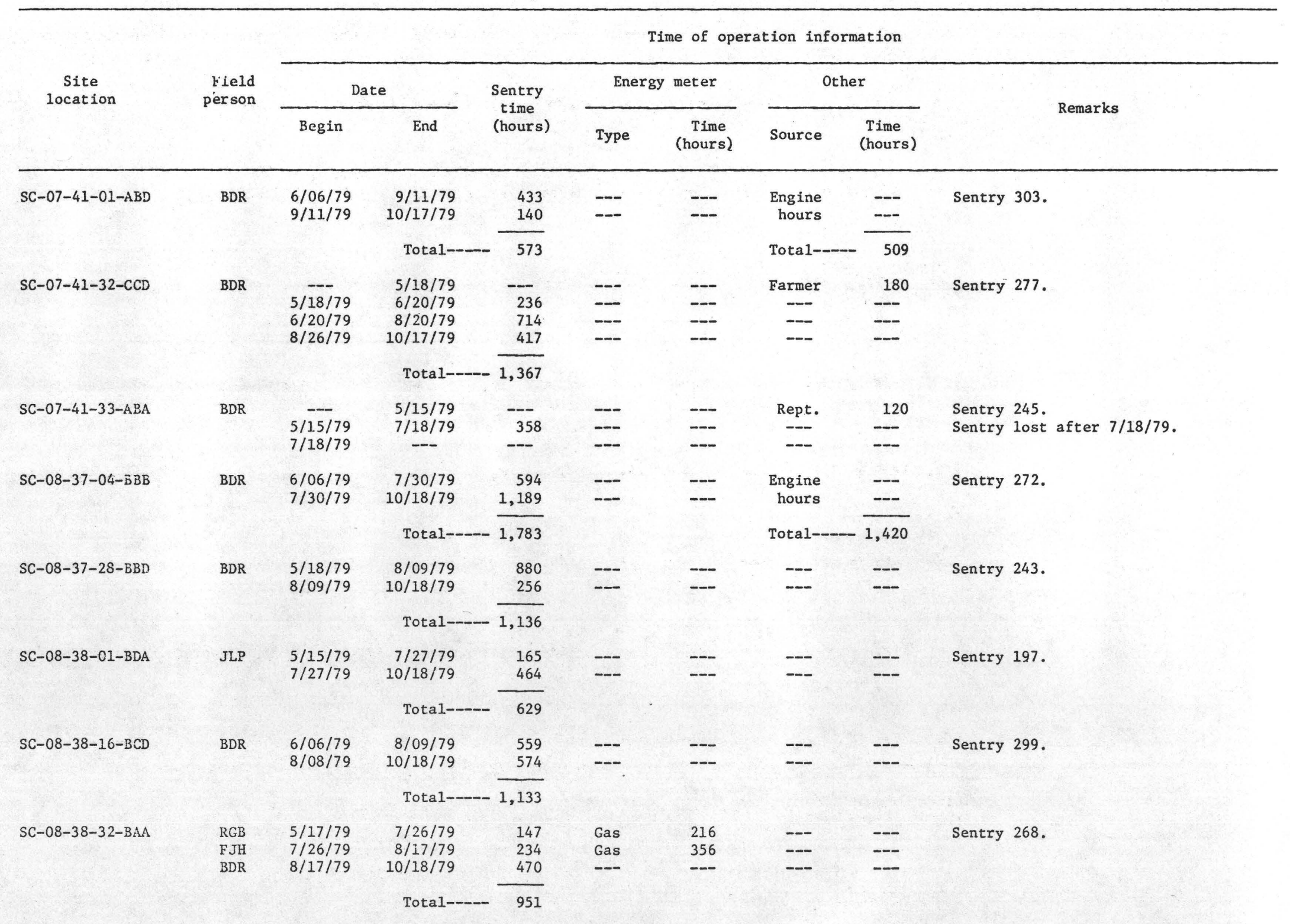


Table 10a.--Pumpage data for irrigation sites in Sherman County, Kansas--Continued

\begin{tabular}{|c|c|c|c|c|c|c|c|c|c|c|c|c|}
\hline \multirow[b]{2}{*}{$\begin{array}{c}\text { Site } \\
\text { location }\end{array}$} & \multirow[b]{2}{*}{$\begin{array}{l}\text { Station } \\
\text { identifier }\end{array}$} & \multicolumn{4}{|c|}{ Irrigated crop information } & \multicolumn{6}{|c|}{ Well information } & \multirow[b]{2}{*}{ Remarks } \\
\hline & & Type & System & Acres & Source & $\begin{array}{r}\text { Depth } \\
(\mathrm{ft})\end{array}$ & $\begin{array}{r}\text { Water } \\
\text { level } \\
\text { (ft) }\end{array}$ & $\begin{array}{l}\text { Diam- } \\
\text { meter } \\
\text { (in) }\end{array}$ & $\begin{array}{l}\text { Pump } \\
\text { hp. }\end{array}$ & $\begin{array}{l}\text { Energy } \\
\text { source }\end{array}$ & $\begin{array}{l}\text { Energy } \\
\text { rating }\end{array}$ & \\
\hline SC-08-39-25-CAB & $-\cdots$ & Corn & Flood & 107 & Rept. & -- & --- & --- & -- & N. gas & 0.32 & \\
\hline$S C-08-39-36-D C D$ & 391830101371201 & Alfalfa & Pivet & 126 & Rept. & -- & -- & -- & -- & N. gas & -- & \\
\hline$S C-08-40-14-D C B$ & ---- & Corn & Flood & 84 & Rept. & -- & -- & -- & -- & N. gas & -- & \\
\hline $\mathrm{SC}-08-40-29-\mathrm{BBB}$ & $-\cdots$ & Corn & Flood & 40 & Rept. & -- & -- & -- & 25 & Elec. & 14.9 & \\
\hline$S C-08-40-31-D A A$ & 391849101491501 & Corn & Pivot & 120 & Rept. & $\cdots$ & -- & -- & -- & N. gas & .14 & \\
\hline$S C-09-37-07-D D B$ & ---- & Alfalfa & Pivot & 130 & Rept. & -- & -- & -- & 100 & Elec. & 59.1 & \\
\hline SC-09-39-01-DBA & 391758101371201 & $\begin{array}{l}\text { Corn } \\
\text { Milo }\end{array}$ & Flood & $\begin{array}{r}117 \\
25\end{array}$ & Rept. & -- & -- & -- & -- & N. gas & --- & \\
\hline $\mathrm{SC}-09-40-28-\mathrm{BAC}$ & $-\cdots$ & Corn & Flood & 160 & Rept. & -- & -- & -- & -- & N. gas & -- & \\
\hline SC-09-40-28-DBA & -..- & Corn & Flood & -- & --- & -- & -- & -- & -- & N. gas & -- & \\
\hline
\end{tabular}


Table 10b.--Prmpage data for irrigation sites in Sherman County, Kansas--Continued

\begin{tabular}{|c|c|c|c|c|c|}
\hline \multirow{2}{*}{$\begin{array}{c}\text { Site } \\
\text { location }\end{array}$} & \multirow{2}{*}{$\begin{array}{r}\text { Field } \\
\text { person }\end{array}$} & \multicolumn{4}{|c|}{ Discharge information } \\
\hline & & Date & $\begin{array}{l}\text { Discharge } \\
\text { (gal/min) }\end{array}$ & Method & Remarks \\
\hline SC-08-39-25-CAB & BDR & $\begin{array}{l}7 / 19 / 79 \\
7 / 19 / 79 \\
7 / 19 / 79\end{array}$ & $\begin{array}{r}695 \\
1,050 \\
660\end{array}$ & $\begin{array}{l}\text { T.T. } \\
\text { Dop. } \\
\text { Prop. }\end{array}$ & Measurement on 8-in aluminum-gated pipe. \\
\hline $\mathrm{SC}-08-39-36-\mathrm{DCD}$ & BDR & $\begin{array}{l}7 / 19 / 79 \\
7 / 19 / 79\end{array}$ & $\begin{array}{l}530 \\
565\end{array}$ & $\begin{array}{l}\text { T.T. } \\
\text { Dop. }\end{array}$ & Measurement on 8-in aluminum pipe. \\
\hline SC-08-40-14-DCB & BDR & $\begin{array}{l}7 / 19 / 79 \\
7 / 19 / 79 \\
7 / 19 / 79\end{array}$ & $\begin{array}{l}505 \\
425 \\
390\end{array}$ & $\begin{array}{l}\text { T.T. } \\
\text { Dop. } \\
\text { Prop. }\end{array}$ & Measurement on 8-in aluminum-gated pipe. \\
\hline $\mathrm{SC}-08-40-29-\mathrm{BBB}$ & BDR & -- & 260 & Rept. & Owner estimate. \\
\hline SC-08-40-31-DAA & $\begin{array}{l}\text { FJH } \\
\text { RGB }\end{array}$ & $\begin{array}{l}8 / 17 / 79 \\
8 / 17 / 79\end{array}$ & $\begin{array}{l}640 \\
625\end{array}$ & $\begin{array}{l}\text { T.T. } \\
\text { Dop. }\end{array}$ & Measurement on 8 -in steel tubing. \\
\hline SC-09-37-07-DDB & $\pi P$ & $7 / 31 / 79$ & 620 & T.T. & Measurement on 8 -in aluminum pipe. \\
\hline SC-09-39-01-DBA & BDR & $\begin{array}{l}8 / 27 / 79 \\
8 / 27 / 79\end{array}$ & $\begin{array}{l}715 \\
485\end{array}$ & $\begin{array}{l}\text { T.T. } \\
\text { Dop. }\end{array}$ & Measurement on 8-in aluminum-gated pipe. \\
\hline$S C-09-40-28-B A C$ & BDR & -- & -- & -- & No discharge; multiple well system. \\
\hline$S C-09-40-28-D B A$ & $\mathrm{BDR}$ & -- & -- & -- & No discharge; multiple well system. \\
\hline
\end{tabular}


Table 10c.--Pumpage data for irrigation sites. in Sherman County, Kansas--Continued

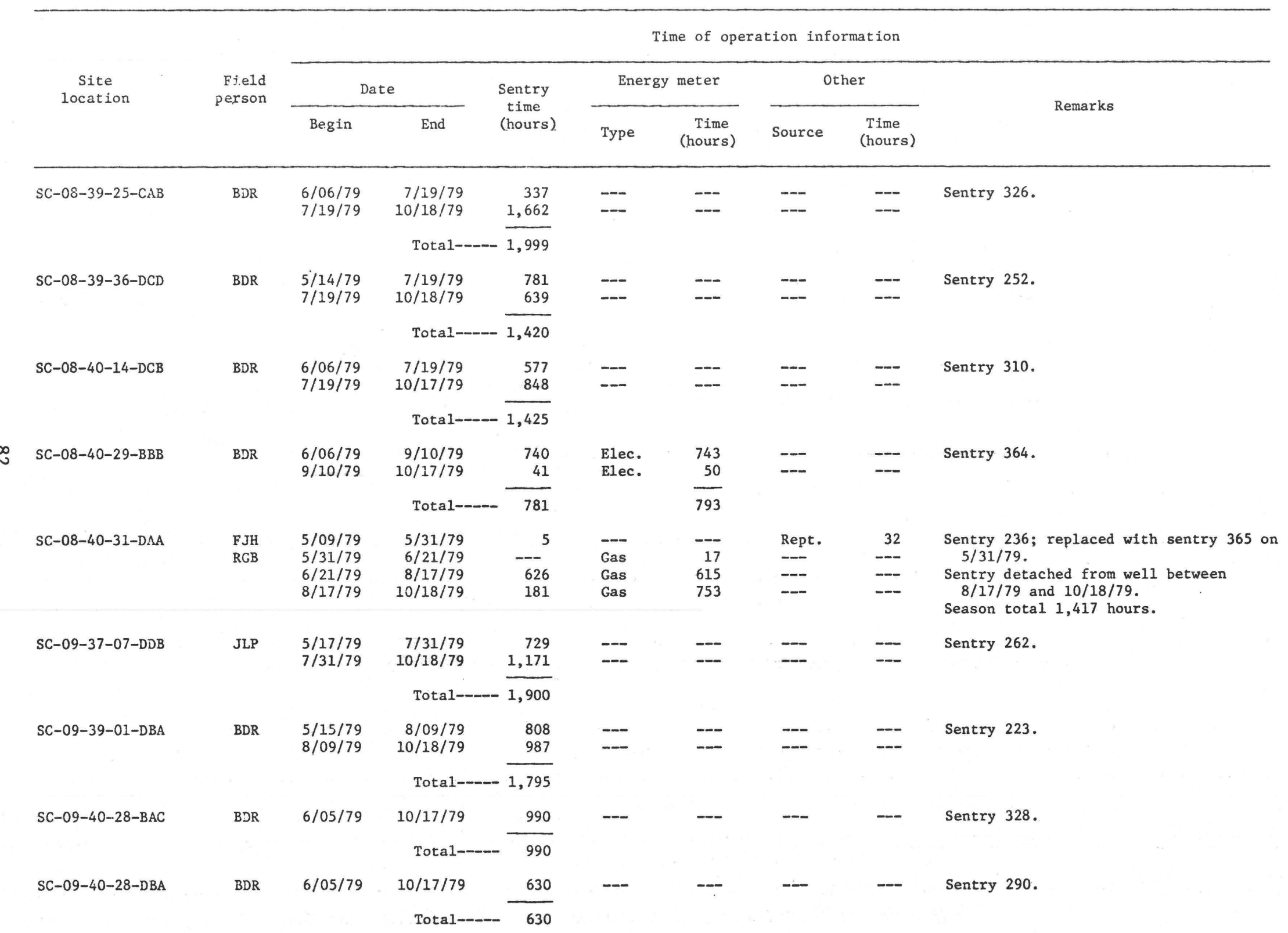


Table 10a.--Pumpage data for irrigation sites in Sherman County, Kansas--Continued

\begin{tabular}{|c|c|c|c|c|c|c|c|c|c|c|c|c|}
\hline \multirow[b]{2}{*}{$\begin{array}{c}\text { Site } \\
\text { location }\end{array}$} & \multirow{2}{*}{$\begin{array}{l}\text { Station } \\
\text { identifier }\end{array}$} & \multicolumn{4}{|c|}{ Irrigated crop information } & \multicolumn{6}{|c|}{ Well information } & \multirow[b]{2}{*}{ Remarks } \\
\hline & & Type & System & Acres & Source & $\begin{array}{l}\text { Depth } \\
(\mathrm{ft})\end{array}$ & $\begin{array}{r}\text { Water } \\
\text { level } \\
\text { (ft) }\end{array}$ & $\begin{array}{l}\text { Diam- } \\
\text { meter } \\
\text { (in) }\end{array}$ & $\begin{array}{l}\text { Pump } \\
\text { hp. }\end{array}$ & $\begin{array}{l}\text { Energy } \\
\text { source }\end{array}$ & $\begin{array}{l}\text { Energy } \\
\text { rating }\end{array}$ & \\
\hline SC-09-40-30-B & 391441101495701 & $\begin{array}{l}\text { Corn } \\
\text { Beans }\end{array}$ & Flood & $\begin{array}{l}40 \\
40\end{array}$ & Rept. & -- & -- & -- & -- & N. gas & -- & \\
\hline SC-09-41-03-DAA & 391756101523301 & $\begin{array}{l}\text { Corn } \\
\text { Beets }\end{array}$ & Flosd & $\begin{array}{l}51 \\
51\end{array}$ & Rept. & --- & -- & --- & -- & N. gas & .14 & \\
\hline SC-09-41-09-CDC & 391645101542301 & Corn & Pivot & 95 & Rept. & --- & -- & -- & -- & N. gas & -- & \\
\hline$S C-09-41-12-B B C$ & 391724101511801 & Corn & Flood & 150 & Rept. & -- & -- & -- & - & N. gas & .13 & \\
\hline SC-09-41-16-BCD & 391618101543201 & Corn & Pivot & 125 & Rept. & -- & -- & -- & -- & N. gas & -- & \\
\hline SC-09-42-16-DCA & 391558102003801 & Corn & Pivot & 123 & Meas. & -- & -- & -- & -- & N. gas & -- & \\
\hline SC-09-42-28-BCC & $\ldots$ & $\begin{array}{c}\text { Corn } \\
\text { Beets } \\
\text { Beans } \\
\text { Alfalfa }\end{array}$ & Flood & $\begin{array}{l}35 \\
36 \\
35 \\
36\end{array}$ & Rept. & -- & -- & -- & -- & N. gas & -- & \\
\hline$S C-10-37-23-A B B$ & $\ldots$ & Corn & Flood & 160 & Rept. & -- & -- & -- & -- & Diese1 & -- & \\
\hline
\end{tabular}


Table 10b.--Pumpage data for irrigation sites in Sherman County, Kansas--Continued

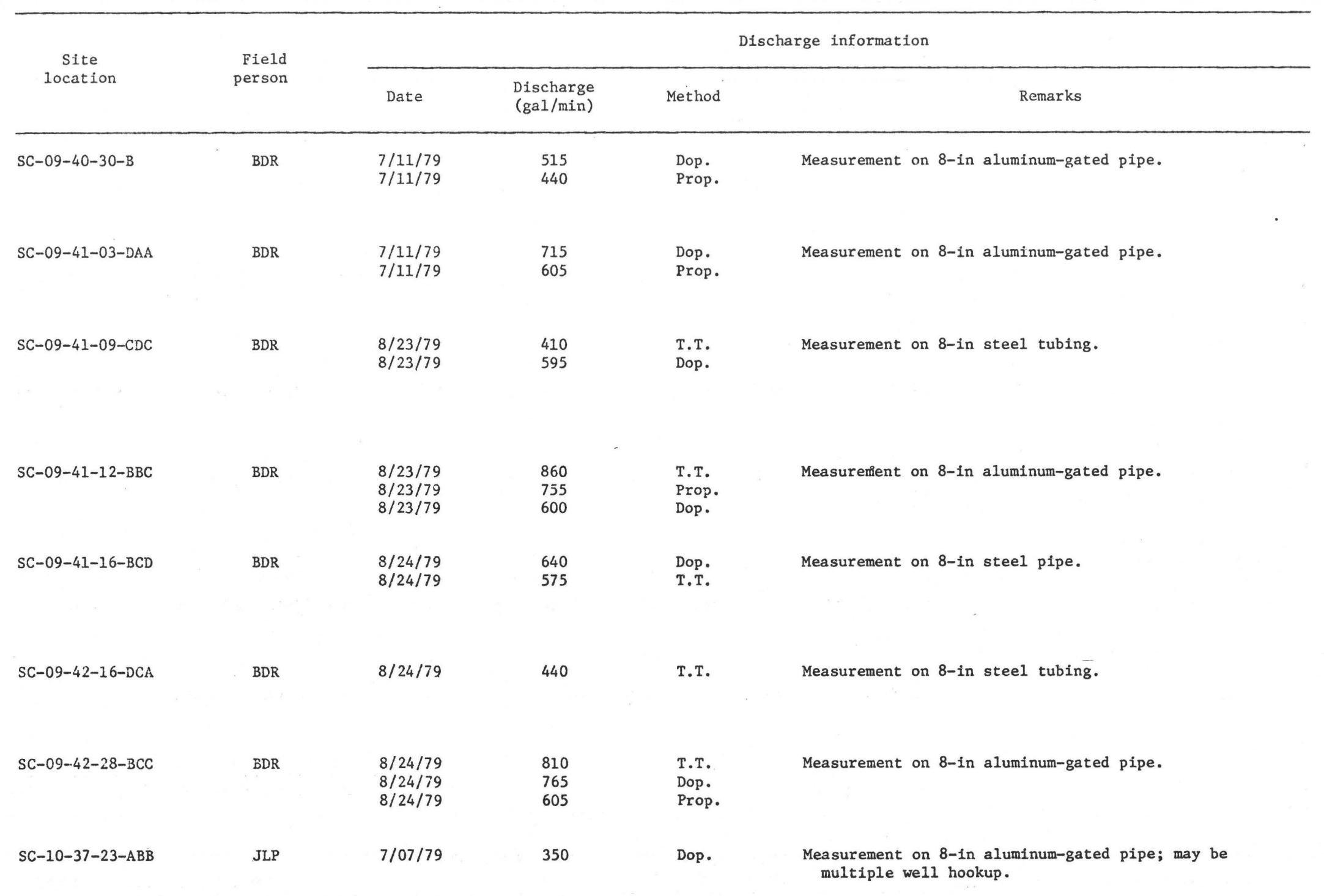


Table 10c.--Pumpage data for irrigation sites in Sherman County, Kansas--Continued

\begin{tabular}{|c|c|c|c|c|c|c|c|c|c|}
\hline \multirow{3}{*}{$\begin{array}{l}\text { Site } \\
\text { location }\end{array}$} & \multirow{3}{*}{$\begin{array}{r}\text { Field } \\
\text { person }\end{array}$} & \multicolumn{8}{|c|}{ Time of operation information } \\
\hline & & \multicolumn{2}{|c|}{ Date } & \multirow{2}{*}{$\begin{array}{l}\text { Sentry } \\
\text { time } \\
\text { (hours) }\end{array}$} & \multicolumn{2}{|c|}{ Energy meter } & \multicolumn{2}{|c|}{ Other } & \multirow{2}{*}{ Remarks } \\
\hline & & Begin & End & & Type & $\begin{array}{c}\text { Time } \\
\text { (hours) }\end{array}$ & Source & $\begin{array}{l}\text { Time } \\
\text { (hours) }\end{array}$ & \\
\hline \multirow[t]{2}{*}{ SC-09-40-30-B } & $\mathrm{BDR}$ & $\begin{array}{l}6 / 05 / 79 \\
7 / 11 / 79\end{array}$ & $\begin{array}{r}7 / 11 / 79 \\
10 / 17 / 79\end{array}$ & $\begin{array}{r}228 \\
1,002\end{array}$ & -- & $\overline{--}$ & - & -- & \multirow[t]{2}{*}{ Sentry 274 . } \\
\hline & & & Total-- & 1,230 & & & & & \\
\hline \multirow[t]{2}{*}{$S C-09-41-03-D A A$} & BDR & $\begin{array}{l}6 / 06 / 79 \\
7 / 11 / 79\end{array}$ & $\begin{array}{r}7 / 11 / 79 \\
10 / 17 / 79\end{array}$ & $\begin{array}{l}203 \\
805\end{array}$ & Gas & 208 & - & - & \multirow[t]{2}{*}{ Sentry 363.} \\
\hline & & & Total-- & 1,008 & & & & & \\
\hline \multirow[t]{2}{*}{ SC-09-41-09-CDC } & $B D R$ & $\begin{array}{l}5 / 08 / 79 \\
6 / 01 / 79 \\
6 / 21 / 79 \\
8 / 23 / 79\end{array}$ & $\begin{array}{r}6 / 01 / 79 \\
6 / 21 / 79 \\
8 / 23 / 79 \\
10 / 17 / 79\end{array}$ & $\begin{array}{r}54 \\
29 \\
942 \\
332 \\
\end{array}$ & $\overline{--}$ & -- & $\overline{--}$ & -- & \multirow[t]{2}{*}{ Sentry 192.} \\
\hline & & & Total-- & 1,357 & & & & & \\
\hline \multirow[t]{2}{*}{$S C-09-41-12-B B C$} & BDR & $\begin{array}{l}5 / 09 / 79 \\
8 / 23 / 79\end{array}$ & $\begin{array}{r}8 / 23 / 79 \\
10 / 17 / 79\end{array}$ & $\begin{array}{l}830 \\
380 \\
\end{array}$ & Gas & 931 & -- & -- & \multirow[t]{2}{*}{$\begin{array}{l}\text { Sentry } 250 \text { replaced with sentry } 499 \\
\text { on } 8 / 23 / 79 \text {. }\end{array}$} \\
\hline & & & Tota1-- & 1,210 & & & & & \\
\hline \multirow[t]{2}{*}{$\mathrm{SC}-09-41-16-\mathrm{BCD}$} & BDR & $\begin{array}{l}5 / 09 / 79 \\
7 / 11 / 79 \\
8 / 24 / 79\end{array}$ & $\begin{array}{r}7 / 11 / 79 \\
8 / 24 / 79 \\
10 / 17 / 79\end{array}$ & $\begin{array}{l}284 \\
786 \\
368\end{array}$ & $\overline{-}$ & -- & $\cdots$ & $\cdots$ & \multirow[t]{2}{*}{ Sentry 276.} \\
\hline & & & Total-- & 1,438 & & & & & \\
\hline \multirow[t]{2}{*}{ SC-09-42-16-DCA } & BDR & $\begin{array}{l}6 / 05 / 79 \\
8 / 24 / 79\end{array}$ & $\begin{array}{r}8 / 24 / 79 \\
10 / 17 / 79\end{array}$ & $\begin{array}{r}1,356 \\
254\end{array}$ & -- & - & - & $\cdots$ & \multirow[t]{2}{*}{ Sentry 372.} \\
\hline & & & Tota1-- & 1,610 & & & & & \\
\hline \multirow[t]{2}{*}{$S C-09-42-28-B C C$} & BDR & $\begin{array}{l}5 / 22 / 79 \\
8 / 24 / 79\end{array}$ & $\begin{array}{r}8 / 24 / 79 \\
10 / 17 / 79\end{array}$ & $\begin{array}{r}1,220 \\
762 \\
\end{array}$ & - & -- & - & - & \multirow[t]{2}{*}{ Sentry 266} \\
\hline & & & Total-- & 1,982 & & & & & \\
\hline$S C-10-37-23-A B B$ & JLP & $\begin{array}{l}5 / 15 / 79 \\
7 / 11 / 79\end{array}$ & $\begin{array}{r}7 / 11 / 79 \\
10 / 17 / 79\end{array}$ & $\begin{array}{r}291 \\
1,458\end{array}$ & -- & $-\cdots$ & - & - & Sentry 232. \\
\hline
\end{tabular}


Table 10a.--Fumpage data for irrigation sites in Sherman County, Kansas--Continued

\begin{tabular}{|c|c|c|c|c|c|c|c|c|c|c|c|c|}
\hline \multirow{2}{*}{$\begin{array}{c}\text { Site } \\
\text { location }\end{array}$} & \multirow{2}{*}{$\begin{array}{l}\text { Station } \\
\text { identifier }\end{array}$} & \multicolumn{4}{|c|}{ Irrigated crop information } & \multicolumn{6}{|c|}{ We11 information } & \multirow{2}{*}{ Remarks } \\
\hline & & Type & System & Acres & Source & $\begin{array}{c}\text { Depth } \\
(\mathrm{ft})\end{array}$ & $\begin{array}{r}\text { Water } \\
\text { level } \\
(\mathrm{ft})\end{array}$ & $\begin{array}{l}\text { Diam- } \\
\text { meter } \\
\text { (in) }\end{array}$ & $\begin{array}{l}\text { Pump } \\
\text { hp. }\end{array}$ & $\begin{array}{l}\text { Energy } \\
\text { source }\end{array}$ & $\begin{array}{l}\text { Energy } \\
\text { rating }\end{array}$ & \\
\hline$S C-10-37-23-C D C$ & $-\cdots$ & Corn & Flood & 400 & Rept. & -- & -- & -- & -- & Diesel & -- & \\
\hline SC-10-42-12-CAA & 391151101573901 & Corn & Flood & 60 & Rept. & -- & -- & -- & -- & N. gas & -- & \\
\hline$S C-10-42-12-A B B$ & $-\cdots$ & Corn & Flood & 160 & Rept. & -- & -- & -- & -- & N. gas & -- & \\
\hline$S C-10-42-17-D B D$ & --- & Corn & Pivot & 120 & Rept. & -- & -- & -- & -- & N. gas & -- & \\
\hline
\end{tabular}


Table 10b.--Pumpage data for irrigation sites in Sherman County, Kansas--Continued

\begin{tabular}{|c|c|c|c|c|c|}
\hline \multirow{2}{*}{$\begin{array}{l}\text { Site } \\
\text { location }\end{array}$} & \multirow{2}{*}{$\begin{array}{r}\text { Field } \\
\text { person }\end{array}$} & \multicolumn{4}{|c|}{ Discharge information } \\
\hline & & Date & $\begin{array}{l}\text { Discharge } \\
\text { (gal/min) }\end{array}$ & Method & Remarks \\
\hline$S C-10-37-23-C D C$ & JLP & $\begin{array}{l}7 / 07 / 79 \\
7 / 07 / 79\end{array}$ & $\begin{array}{l}735 \\
510\end{array}$ & $\begin{array}{l}\text { Dop. } \\
\text { Prop. }\end{array}$ & $\begin{array}{l}\text { Measurement on } 6 \text {-in aluminum-gated pipe. } \\
\text { May be multiple well hookup. }\end{array}$ \\
\hline SC-10-42-12-CAA & BDR & $\begin{array}{l}7 / 25 / 79 \\
7 / 25 / 79 \\
7 / 25 / 79\end{array}$ & $\begin{array}{l}515 \\
655 \\
970\end{array}$ & $\begin{array}{l}\text { T.T. } \\
\text { Prop. } \\
\text { Dop. }\end{array}$ & Measurement on 6-in aluminum-gated pipe. \\
\hline$S C-10-42-12-A B B$ & BDR & $\begin{array}{l}7 / 25 / 79 \\
7 / 25 / 79\end{array}$ & $\begin{array}{l}685 \\
470\end{array}$ & $\begin{array}{l}\text { Dop. } \\
\text { Prop. }\end{array}$ & Measurement on 10-in aluminum-gated pipe. \\
\hline$S C-10-42-17-D B D$ & BDR & $8 / 27 / 79$ & 640 & T.T. & Measurement on 8-in steel tubing. \\
\hline
\end{tabular}


Table 10c.--Pumpage data for irrigation sites in Sherman County, Kansas--Continued

\begin{tabular}{|c|c|c|c|c|c|c|c|c|c|c|}
\hline \multirow{3}{*}{$\begin{array}{l}\text { Site } \\
\text { location }\end{array}$} & \multirow{3}{*}{$\begin{array}{l}\text { Field } \\
\text { person }\end{array}$} & \multicolumn{9}{|c|}{ Time of operation information } \\
\hline & & \multicolumn{2}{|c|}{ Date } & \multirow{2}{*}{$\begin{array}{l}\text { Sentry } \\
\text { time } \\
\text { (hours) }\end{array}$} & \multicolumn{2}{|c|}{ Energy meter } & \multicolumn{2}{|c|}{ Other } & \multirow{2}{*}{\multicolumn{2}{|c|}{ Remarks }} \\
\hline & & Begin & End & & Type & $\begin{array}{c}\text { Time } \\
\text { (hours) }\end{array}$ & Source & $\begin{array}{l}\text { Time } \\
\text { (hours) }\end{array}$ & & \\
\hline$S C-10-37-23-C D C$ & JLP & $\begin{array}{l}5 / 15 / 79 \\
7 / 07 / 79\end{array}$ & $\begin{array}{r}7 / 07 / 79 \\
10 / 17 / 79\end{array}$ & $\begin{array}{r}316 \\
1,405\end{array}$ & -- & -- & -- & $-\infty$ & \multicolumn{2}{|c|}{ Sentry 222.} \\
\hline \multirow[t]{2}{*}{$\mathrm{SC}-10-42-12-\mathrm{CAA}$} & BDR & $\begin{array}{l}5 / 09 / 79 \\
7 / 25 / 79\end{array}$ & $\begin{array}{r}\text { Tota1- } \\
7 / 25 / 79 \\
10 / 17 / 79\end{array}$ & $\begin{array}{r}-1,721 \\
392 \\
667 \\
\end{array}$ & \multirow[t]{2}{*}{--} & \multirow[t]{2}{*}{--} & \multirow[t]{2}{*}{--} & \multirow[t]{2}{*}{--} & \multicolumn{2}{|c|}{ Sentry 227.} \\
\hline & & & Total- & 1,059 & & & & & & \\
\hline$S C-10-42-12-A B B$ & $\mathrm{BDR}$ & $\begin{array}{l}5 / 17 / 79 \\
7 / 25 / 79\end{array}$ & $\begin{array}{r}7 / 25 / 79 \\
10 / 17 / 79\end{array}$ & $\begin{array}{l}339 \\
966\end{array}$ & -- & $\cdots$ & $-\infty$ & -- & Sentry & 269. \\
\hline \multirow[t]{2}{*}{$S C-10-42-17-D B D$} & $\mathrm{BDR}$ & $\begin{array}{l}6 / 06 / 79 \\
8 / 27 / 79\end{array}$ & $\begin{array}{r}\text { Total- } \\
8 / 27 / 79 \\
10 / 17 / 79\end{array}$ & $\begin{array}{r}1,305 \\
971 \\
214\end{array}$ & \multirow[t]{2}{*}{ Gas } & \multirow[t]{2}{*}{$\begin{array}{l}881 \\
---\end{array}$} & \multirow[t]{2}{*}{-} & \multirow[t]{2}{*}{$-\infty$} & \multirow[t]{2}{*}{ Sentry } & 330. \\
\hline & & & Total-- & 1,185 & & & & & & \\
\hline
\end{tabular}


Table 11a.--Pumpage data for irrigation sites in Chase County, Nebraska

\begin{tabular}{|c|c|c|c|c|c|c|c|c|c|c|c|c|}
\hline \multirow{2}{*}{$\begin{array}{c}\text { Site } \\
\text { location }\end{array}$} & \multirow{2}{*}{$\begin{array}{l}\text { Station } \\
\text { identifier }\end{array}$} & \multicolumn{4}{|c|}{ Irrigated crop information } & \multicolumn{6}{|c|}{ We11 information } & \multirow{2}{*}{ Remarks } \\
\hline & & Type & System & Acres & Source & $\begin{array}{l}\text { Depth } \\
\text { (ft) }\end{array}$ & $\begin{array}{r}\text { Water } \\
\text { level } \\
\text { (ft) }\end{array}$ & $\begin{array}{l}\text { Diam- } \\
\text { meter } \\
\text { (in) }\end{array}$ & $\begin{array}{c}\text { Pump } \\
\text { hp. }\end{array}$ & $\begin{array}{l}\text { Energy } \\
\text { source }\end{array}$ & $\begin{array}{l}\text { Energy } \\
\text { rating }\end{array}$ & \\
\hline$S B-05-38-31-C C$ & 402108101404901 & Corn & Flood & 80 & Meas. & 298 & -- & 18 & -- & N. gas & -- & \\
\hline$S B-05-39-08-C D$ & 402437101462301 & Alfalfa & $\begin{array}{c}\text { Sprink- } \\
\text { ler }\end{array}$ & 122 & Rept. & -- & -- & -- & -- & Elec. & -- & \\
\hline$S B-05-39-21-B B$ & 402325101452301 & Corn & Pivot & 127 & Rept. & 320 & -- & 16 & -- & Diese1 & -- & \\
\hline$S B-05-40-01-C$ & 402350101531701 & Corn & Pivot & 140 & Rept. & -- & -- & -- & -- & Elec. & -- & \\
\hline$S B-06-38-05-C C$ & $-\cdots$ & Alfalfa & Pivot & 32 & Rept. & -- & - & -- & - & N. gas & -- & \\
\hline$S B-06-40-09-D A$ & 403001101512501 & $\begin{array}{l}\text { Alfalfa } \\
\text { Beans }\end{array}$ & Pivot & $\begin{array}{l}79 \\
79\end{array}$ & Meas. & -- & -- & -- & -- & Elec. & -- & \\
\hline$S B-06-40-30-A D$ & 402738101534301 & Corn & Pivot & 106 & Meas. & 340 & - & 16 & -- & N. gas & - & \\
\hline$S B-06-41-25-D D$ & 402711101545201 & Corn & Pivot & 200 & Rept. & 315 & -- & 16 & - & N. gas & -- & \\
\hline
\end{tabular}


Table 11b.--Pumpage data for irrigation sites in Chase Couniy, Nebraska

\begin{tabular}{|c|c|c|c|c|c|}
\hline \multirow{2}{*}{$\begin{array}{c}\text { Site } \\
\text { location }\end{array}$} & \multirow{2}{*}{$\begin{array}{l}\text { Field } \\
\text { person }\end{array}$} & \multicolumn{4}{|c|}{ Discharge information } \\
\hline & & Date & $\begin{array}{l}\text { Discharge } \\
\text { (gal/min) }\end{array}$ & Method & Remarks \\
\hline $\mathrm{SC}-05-38-31-\mathrm{CC}$ & JLP & $\begin{array}{l}7 / 23 / 79 \\
7 / 23 / 79 \\
7 / 23 / 79\end{array}$ & $\begin{array}{r}2,865 \\
2,135 \\
860\end{array}$ & $\begin{array}{l}\text { Dop. } \\
\text { Prop. } \\
\text { Dop. }\end{array}$ & $\begin{array}{l}\text { Measurement on } 10 \text {-in aluminum-gated pipe. } \\
\text { Polysonics meter. }\end{array}$ \\
\hline$S B-05-39-08-C D$ & JLP & $\begin{array}{l}8 / 09 / 79 \\
8 / 09 / 79 \\
8 / 09 / 79\end{array}$ & $\begin{array}{l}765 \\
715 \\
750\end{array}$ & $\begin{array}{l}\text { T.T. } \\
\text { Dop. } \\
\text { In1. }\end{array}$ & Measurement on 8 -in steel pipe. \\
\hline$S B-05-39-21-B B$ & JLP & $\begin{array}{l}7 / 20 / 79 \\
7 / 20 / 79 \\
7 / 20 / 79\end{array}$ & $\begin{array}{l}995 \\
370 \\
950\end{array}$ & $\begin{array}{l}\text { T.T. } \\
\text { Dop. } \\
\text { In1. }\end{array}$ & Measurement on 8-in steel tubing. \\
\hline$S B-05-40-01-C$ & JLP & $\begin{array}{l}7 / 21 / 79 \\
7 / 21 / 79 \\
7 / 21 / 79\end{array}$ & $\begin{array}{l}950 \\
595 \\
900\end{array}$ & $\begin{array}{l}\text { T.T. } \\
\text { Dop. } \\
\text { Inl. }\end{array}$ & Measurement on 8 -in steel tubing. \\
\hline $\mathrm{SB}-06-38-05-\mathrm{CC}$ & JLP & $\begin{array}{l}7 / 19 / 79 \\
7 / 19 / 79\end{array}$ & $\begin{array}{l}280 \\
350\end{array}$ & $\begin{array}{l}\text { T.T. } \\
\text { Dop. }\end{array}$ & Measurement on 8 -in aluminum pipe. \\
\hline SB-06-40-09-DA & CEP & $6 / 13 / 79$ & 800 & T.T. & Measurement on 8 -in steel tubing. \\
\hline$S B-06-40-30-A D$ & $\begin{array}{l}\text { CEP } \\
\text { RRL }\end{array}$ & $\begin{array}{l}8 / 17 / 79 \\
8 / 17 / 79\end{array}$ & $\begin{array}{l}635 \\
150\end{array}$ & $\begin{array}{l}\text { T.T. } \\
\text { Dop. }\end{array}$ & Measurement on 6-in steel pipe. \\
\hline SB-06-41-25-DD & JLP & $\begin{array}{r}7 / 12 / 79 \\
7 / 12 / 79 \\
\text { To }\end{array}$ & $\begin{array}{r}655 \\
500 \\
1,155\end{array}$ & $\begin{array}{l}\text { T.T. } \\
\text { Dop. }\end{array}$ & $\begin{array}{l}\text { Well discharges to } 2 \text { pipes; } 8 \text {-in steel tubing, one } \\
\text { measured with each method. } \\
\text { Total is total discharge from well. }\end{array}$ \\
\hline & $\begin{array}{l}\text { CEP } \\
\text { RRL }\end{array}$ & $\begin{array}{l}8 / 17 / 79 \\
8 / 17 / 79\end{array}$ & $\begin{array}{l}665 \\
455\end{array}$ & $\begin{array}{l}\text { T.T. } \\
\text { T.T. }\end{array}$ & $\begin{array}{l}\text { Same as measurement on } 7 / 12 / 79 \text {, only T.T. method used on } \\
\text { both pipes. }\end{array}$ \\
\hline
\end{tabular}


Table 11c.--Pumpage data for irrigation. sites in Chase County, Nebraska

\begin{tabular}{|c|c|c|c|c|c|c|c|c|c|}
\hline \multirow{3}{*}{$\begin{array}{l}\text { Site } \\
\text { location }\end{array}$} & \multirow{3}{*}{$\begin{array}{r}\text { Field } \\
\text { person }\end{array}$} & \multicolumn{8}{|c|}{ Time of operation information } \\
\hline & & \multicolumn{2}{|c|}{ Date } & \multirow{2}{*}{$\begin{array}{l}\text { Sentry } \\
\text { time } \\
\text { (hours) }\end{array}$} & \multicolumn{2}{|c|}{ Energy meter } & \multicolumn{2}{|c|}{ Other } & \multirow{2}{*}{ Remarks } \\
\hline & & Begin & End & & Type & $\begin{array}{c}\text { Time } \\
\text { (hours) }\end{array}$ & Source & $\begin{array}{l}\text { Time } \\
\text { (hours) }\end{array}$ & \\
\hline \multirow[t]{2}{*}{$S B-05-38-31-C C$} & JLP & $\begin{array}{l}4 / 18 / 79 \\
7 / 23 / 79\end{array}$ & $\begin{array}{l}7 / 23 / 79 \\
9 / 18 / 79\end{array}$ & $\begin{array}{r}65 \\
212\end{array}$ & -- & -- & -- & -- & Sentry 199. Sentry time seems low. \\
\hline & & & Total-- & 277 & & & & & \\
\hline \multirow[t]{2}{*}{$S B-05-39-08-C D$} & JLP & $\begin{array}{l}5 / 03 / 79 \\
8 / 09 / 79\end{array}$ & $\begin{array}{r}8 / 09 / 79 \\
10 / 22 / 79\end{array}$ & $\begin{array}{l}772 \\
494\end{array}$ & -- & -- & -- & -- & Sentry 196. \\
\hline & & & Total-- & 1,266 & & & & & \\
\hline \multirow[t]{2}{*}{$S B-05-39-21-B B$} & JLP & $\begin{array}{l}4 / 09 / 79 \\
7 / 20 / 79\end{array}$ & $\begin{array}{l}7 / 20 / 79 \\
9 / 18 / 79\end{array}$ & $\begin{array}{l}311 \\
996 \\
\end{array}$ & -- & -- & -- & -- & Sentry 212 \\
\hline & & & Total-- & 1,307 & & & & & \\
\hline \multirow[t]{2}{*}{$S B-05-40-01-C$} & JLP & $\begin{array}{l}4 / 20 / 79 \\
7 / 21 / 79\end{array}$ & $\begin{array}{l}7 / 21 / 79 \\
9 / 18 / 79\end{array}$ & $\begin{array}{l}272 \\
527 \\
\end{array}$ & -- & -- & -- & -- & Sentry 211. \\
\hline & & & Tota1-- & 799 & & & & & \\
\hline \multirow[t]{2}{*}{$S B-06-38-05-C C$} & JLP & $\begin{array}{l}4 / 26 / 79 \\
7 / 19 / 79\end{array}$ & $\begin{array}{r}7 / 19 / 79 \\
11 / 02 / 75\end{array}$ & $\begin{array}{l}388 \\
855 \\
\end{array}$ & $\cdots$ & -- & -- & -- & Sentry 190. \\
\hline & & & Total-- & 1,243 & & & & & \\
\hline$S B-06-40-09-D A$ & CEP & $\begin{array}{l}4 / 27 / 79 \\
6 / 13 / 79\end{array}$ & $\begin{array}{l}6 / 13 / 79 \\
9 / 18 / 79\end{array}$ & - & $\overline{-\cdots}$ & - & -- & -- & $\begin{array}{l}\text { Sentry } 225 . \\
\text { Sentry lost from site. }\end{array}$ \\
\hline \multirow[t]{2}{*}{$S B-06-40-30-A D$} & $\begin{array}{l}\text { CEP } \\
\text { RRL }\end{array}$ & $\begin{array}{l}4 / 21 / 79 \\
6 / 13 / 79 \\
8 / 17 / 79\end{array}$ & $\begin{array}{l}6 / 13 / 79 \\
8 / 17 / 79 \\
9 / 18 / 79\end{array}$ & $\begin{array}{r}26 \\
441 \\
110\end{array}$ & -- & $\overline{--}$ & -- & -- & Sentry 194. \\
\hline & & & Total-- & 577 & & & & & \\
\hline $\mathrm{SB}-06-41-25-\mathrm{DD}$ & $\begin{array}{l}\text { JLP } \\
\text { CEP } \\
\text { RRL }\end{array}$ & $\begin{array}{l}4 / 09 / 79 \\
7 / 12 / 79 \\
8 / 17 / 79\end{array}$ & $\begin{array}{l}7 / 12 / 79 \\
8 / 17 / 79 \\
9 / 18 / 79\end{array}$ & $\begin{array}{r}219 \\
662 \\
1\end{array}$ & - & -- & -- & -- & $\begin{array}{l}\text { Sentry } 264 . \text { Final time may be low, } \\
\text { but corn was cut early. }\end{array}$ \\
\hline
\end{tabular}


Table 1la.--Pumpage data for irrigation sites in Chase County, Nebraska--Continued

\begin{tabular}{|c|c|c|c|c|c|c|c|c|c|c|c|c|}
\hline \multirow{2}{*}{$\begin{array}{c}\text { Site } \\
\text { location }\end{array}$} & \multirow{2}{*}{$\begin{array}{l}\text { Station } \\
\text { identifier }\end{array}$} & \multicolumn{4}{|c|}{ Irrigated crop information } & \multicolumn{6}{|c|}{ Well information } & \multirow[b]{2}{*}{ Remarks } \\
\hline & & Type & System & Acres & Source & $\begin{array}{c}\text { Depth } \\
(\mathrm{ft})\end{array}$ & $\begin{array}{r}\text { Water } \\
\text { leve1 } \\
(\mathrm{ft})\end{array}$ & $\begin{array}{l}\text { Diam- } \\
\text { meter } \\
\text { (in) }\end{array}$ & $\begin{array}{l}\text { Pump } \\
\text { hp. }\end{array}$ & $\begin{array}{l}\text { Energy } \\
\text { source }\end{array}$ & $\begin{array}{l}\text { Energy } \\
\text { rating }\end{array}$ & \\
\hline SB-06-41-35-CB & 402632101565201 & Corn & Pivot & 146 & Meas. & 300 & -- & 16 & --- & Elec. & -- & \\
\hline $\mathrm{SB}-07-38-31-\mathrm{AC}$ & 403200101401701 & Alfalfa & Pivot & 235 & Rept. & 305 & --- & 16 & 150 & Elec. & 115.2 & \\
\hline SB-07-39-02-CD & 403552101424901 & Corn & Pivot & 140 & Rept. & 288 & -- & 18 & -- & N. gas & -- & \\
\hline $\mathrm{SB}-07-39-02-\mathrm{BB}$ & 403632101430001 & Corn & Pivot & 140 & Rept. & 232 & -- & 16 & -- & N. gas & -- & \\
\hline SB-07-39-07-CB & 403513101474001 & Beets & Pivot & 130 & Rept. & -- & -- & -- & 75 & Elec. & --- & \\
\hline SB-07-39-26-CC & 403223101430601 & $\begin{array}{l}\text { Corn } \\
\text { Beets }\end{array}$ & Flood & $\begin{array}{l}65 \\
65\end{array}$ & Rept. & 235 & -- & -- & -- & Elec. & -- & \\
\hline$S B-07-40-29-A C$ & 403251101525101 & Corn & Flood & 148 & Rept. & 320 & --- & -- & -- & Elec. & .82 & \\
\hline$S B-07-40-30-B A$ & 403304101541701 & Corn & Pivot & 200 & Rept. & -- & --- & -- & -- & Diesel & - & \\
\hline
\end{tabular}


Table 11b.--Pumpage data for irrigation sites in Chase County, Nebraska--Continued

\begin{tabular}{|c|c|c|c|c|c|}
\hline \multirow{2}{*}{$\begin{array}{l}\text { Site } \\
\text { location }\end{array}$} & \multirow{2}{*}{$\begin{array}{r}\text { Field } \\
\text { person }\end{array}$} & \multicolumn{4}{|c|}{ Discharge information } \\
\hline & & Date & $\begin{array}{l}\text { Discharge } \\
\text { (gal/min) }\end{array}$ & Method & Remarks \\
\hline$S B-06-41-35-C B$ & CEP & $7 / 25 / 79$ & 895 & T.T. & Measurement on 8 -in steel tubing. \\
\hline$S B-07-38-31-A C$ & JLP & $\begin{array}{l}7 / 18 / 79 \\
7 / 18 / 79 \\
7 / 18 / 79\end{array}$ & $\begin{array}{r}1,415 \\
875 \\
1,000\end{array}$ & $\begin{array}{l}\text { T.T. } \\
\text { Dop. } \\
\text { In1. }\end{array}$ & Measurement on 8-in steel pipe. \\
\hline$S B-07-39-02-C D$ & JLP & $\begin{array}{l}7 / 18 / 79 \\
7 / 18 / 79\end{array}$ & $\begin{array}{r}1,000 \\
835\end{array}$ & $\begin{array}{l}\text { T.T. } \\
\text { Dop. }\end{array}$ & Measurement on 8 -in aluminum pipe. \\
\hline$S B-07-39-02-B B$ & JLP & $\begin{array}{l}7 / 19 / 79 \\
7 / 19 / 79\end{array}$ & $\begin{array}{l}585 \\
865\end{array}$ & $\begin{array}{l}\text { T.T. } \\
\text { Dop. }\end{array}$ & Measurement on 8 -in aluminum pipe. \\
\hline$S B-07-39-07-C B$ & CEP & $7 / 20 / 79$ & 745 & T.T. & Measurement on 8 -in steel tubing. \\
\hline$S B-07-39-26-C C$ & $\begin{array}{l}\text { JLP } \\
\text { CEP }\end{array}$ & $\begin{array}{l}7 / 18 / 79 \\
7 / 18 / 79 \\
7 / 19 / 79 \\
7 / 19 / 79\end{array}$ & $\begin{array}{l}1,355 \\
1,115 \\
1,320 \\
1,120\end{array}$ & $\begin{array}{l}\text { T.T. } \\
\text { In1. } \\
\text { T.T. } \\
\text { In1. }\end{array}$ & Measurement on 8 -in aluminum pipe. \\
\hline$S B-07-40-29-A C$ & CEP & $\begin{array}{l}7 / 19 / 79 \\
7 / 19 / 79\end{array}$ & $\begin{array}{l}1,935 \\
2,150\end{array}$ & $\begin{array}{l}\text { T.T. } \\
\text { Inl. }\end{array}$ & Measurement on 10 -in steel tubing. \\
\hline$S B-07-40-30-B A$ & CEP & $8 / 13 / 79$ & 780 & T.T. & Mea surement on 8 -in steel pipe. \\
\hline
\end{tabular}


Table 11c.--Fumpage data for irrigation sites in Chase County, Nebraska--Continued

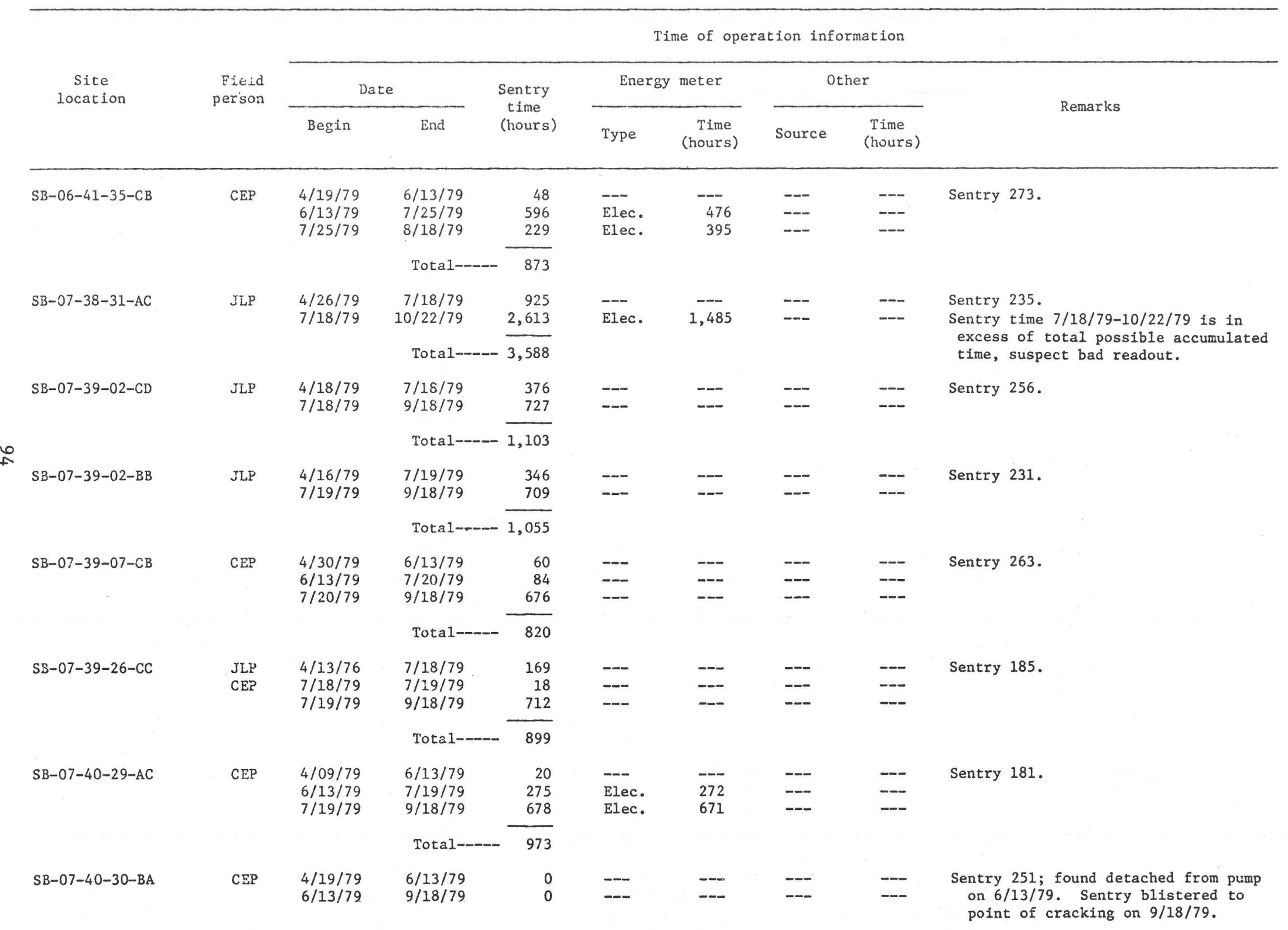


Table 1la.--Pumpage data for irrigation sites in Chase County, Nebraska--Continued

\begin{tabular}{|c|c|c|c|c|c|c|c|c|c|c|c|c|}
\hline \multirow{2}{*}{$\begin{array}{l}\text { Site } \\
\text { location }\end{array}$} & \multirow{2}{*}{$\begin{array}{l}\text { Station } \\
\text { identifier }\end{array}$} & \multicolumn{4}{|c|}{ Irrigated crop information } & \multicolumn{6}{|c|}{ Well information } & \multirow{2}{*}{ Remarks } \\
\hline & & Type & System & Acres & Source & $\begin{array}{l}\text { Depth } \\
(\mathrm{ft})\end{array}$ & $\begin{array}{r}\text { Water } \\
\text { level } \\
\text { (ft) }\end{array}$ & $\begin{array}{l}\text { Diam- } \\
\text { meter } \\
\text { (in) }\end{array}$ & $\begin{array}{l}\text { Pump } \\
\text { hp. }\end{array}$ & $\begin{array}{l}\text { Energy } \\
\text { source }\end{array}$ & $\begin{array}{l}\text { Energy } \\
\text { rating }\end{array}$ & \\
\hline$S B-07-40-34-B B$ & 403012101510701 & Corn & Flood & 50 & Rept. & 310 & -- & 18 & -- & N. gas & -- & \\
\hline$S B-07-41-26-A D$ & 403257101560901 . & Corn & Pivot & 130 & Rept. & 310 & -- & 16 & -- & Elec. & 69.7 & \\
\hline$S B-07-41-34-C$ & 403139101575201 & Corn & Pivot & 115 & Rept. & 282 & -- & 18 & -- & N. gas & -- & \\
\hline$S B-08-36-31-B$ & 403721101270001 & Corn & Pivot & 129 & Rept. & -- & -- & -- & -- & Diesel & -- & \\
\hline$S B-08-38-06-A A$ & 404146101464901 & Corn & Pivot & 125 & Rept. & 330 & -- & 16 & -- & Diese1 & -- & \\
\hline$S B-08-38-34-A D$ & 403713101363601 & Corn & Pivot & 130 & Rept. & 201 & -- & 18 & -- & Diese1 & $\cdots$ & \\
\hline SB-08-39-07-B & 404047101473201 & Corn & Pivot & 128 & Rept. & 298 & -- & 16 & 125 & Elec. & .69 & \\
\hline $\begin{array}{l}S B-08-41-14-C A \\
\text { SB-08-41-14-CC }\end{array}$ & 403935101563501 & $\begin{array}{l}\text { Corn } \\
\text { Beets } \\
\text { Beans }\end{array}$ & Flood & $\begin{array}{l}130 \\
130 \\
130\end{array}$ & $\begin{array}{l}\text { Rept. } \\
\text { Rept. } \\
\text { Rept. }\end{array}$ & -- & -- & -- & -- & -- & -- & \\
\hline
\end{tabular}


Table 11b.--Pumpage data for irrigation sites in Chase County, Nebraska--Continued

\begin{tabular}{|c|c|c|c|c|c|}
\hline \multirow{2}{*}{$\begin{array}{l}\text { Site } \\
\text { location }\end{array}$} & \multirow{2}{*}{$\begin{array}{r}\text { Field } \\
\text { person }\end{array}$} & \multicolumn{4}{|c|}{ Discharge information } \\
\hline & & Date & $\begin{array}{l}\text { Discharge } \\
\text { (gal/min) }\end{array}$ & Method & Remarks \\
\hline$S B-07-40-34-B B$ & CEP & $7 / 19 / 79$ & 840 & Other & $\begin{array}{l}\text { Measurement made in ditch, } 30 \mathrm{ft} \text { from pipe, } \\
\text { with Pygmy meter. }\end{array}$ \\
\hline$S B-07-41-26-A D$ & JLP & $\begin{array}{l}7 / 12 / 79 \\
7 / 12 / 79 \\
7 / 12 / 79\end{array}$ & $\begin{array}{r}895 \\
1,040 \\
800\end{array}$ & $\begin{array}{l}\text { T.T. } \\
\text { Dop. } \\
\text { Inl. }\end{array}$ & Measurement on 8 -in steel tubing. \\
\hline$S B-07-41-34-C$ & CEP & $\begin{array}{l}6 / 13 / 79 \\
8 / 25 / 79\end{array}$ & $\overline{700}$ & T.T. & $\begin{array}{l}\text { Too much turbulance for Clampitron measurement. } \\
\text { Reported estimate. }\end{array}$ \\
\hline$S B-08-36-31-B$ & CEP & $6 / 14 / 79$ & 1,100 & T.T. & Measurement.on 8-in steel pipe. \\
\hline $\mathrm{SB}-08-38-06-\mathrm{AA}$ & CEP & $8 / 27 / 79$ & 690 & T.T. & Measurement on 8-in steel pipe. \\
\hline $\mathrm{SB}-08-38-34-\mathrm{AD}$ & CEP & $8 / 27 / 79$ & 915 & T. T. & Measurement on 8 -in steel tubing. \\
\hline$S B-08-39-07-B$ & CEP & $8 / 27 / 79$ & 530 & T.T. & Measurement on 8-in aluminum-gated pipe. \\
\hline $\begin{array}{l}\text { SB-08-41-14-CA } \\
\text { SB-08-41-14-CC }\end{array}$ & $\begin{array}{l}\text { JLP } \\
\text { CEP }\end{array}$ & $\begin{array}{l}7 / 19 / 79 \\
8 / 18 / 79 \\
8 / 18 / 79 \\
8 / 18 / 79\end{array}$ & $\begin{array}{l}770 \\
700 \\
520 \\
880\end{array}$ & $\begin{array}{l}\text { Traj. } \\
\text { Inl. } \\
\text { Dop. } \\
\text { Other }\end{array}$ & $\begin{array}{l}\text { Measurement on } 10-\text { in aluminum pipe. } \\
\text { Discharge is for we11 SB-08-41-14-CA only; } \\
\text { we11 SB-08-41-14-CC not measured. } \\
\text { Other = pygmy meter used in open ditch. }\end{array}$ \\
\hline
\end{tabular}


Table 11c.--Pumpage data for irrigation sites in Chase County, Nebraska--Continued

\begin{tabular}{|c|c|c|c|c|c|c|c|c|c|}
\hline \multirow{3}{*}{$\begin{array}{l}\text { Site } \\
\text { location }\end{array}$} & \multirow{3}{*}{$\begin{array}{r}\text { Field } \\
\text { person }\end{array}$} & \multicolumn{8}{|c|}{ Time of operation information } \\
\hline & & \multicolumn{2}{|c|}{ Date } & \multirow{2}{*}{$\begin{array}{l}\text { Sentry } \\
\text { time } \\
\text { (hours) }\end{array}$} & \multicolumn{2}{|c|}{ Energy meter } & \multicolumn{2}{|c|}{ Other } & \multirow{2}{*}{ Remarks } \\
\hline & & Begin & End & & Type & $\begin{array}{c}\text { Time } \\
\text { (hours) }\end{array}$ & Source & $\begin{array}{l}\text { Time } \\
\text { (hours) }\end{array}$ & \\
\hline \multirow[t]{2}{*}{$S B-07-40-34-B B$} & CEP & $\begin{array}{l}4 / 19 / 79 \\
7 / 19 / 79\end{array}$ & $\begin{array}{l}7 / 19 / 79 \\
9 / 18 / 79\end{array}$ & $\overline{894}$ & $\begin{array}{l}\text { Gas } \\
\text { Gas }\end{array}$ & $\begin{array}{l}202 \\
722 \\
\end{array}$ & -- & -- & \multirow[t]{2}{*}{$\begin{array}{l}\text { Sentry 206. Time of } 894 \text { hours for } P \\
4 / 19 / 79-9 / 18 / 79\end{array}$} \\
\hline & & & Total--.- & 894 & & 924 & & & \\
\hline$S B-07-41-26-A D$ & JLP & $\begin{array}{l}4 / 13 / 79 \\
7 / 12 / 79\end{array}$ & $\begin{array}{l}7 / 12 / 79 \\
9 / 27 / 79\end{array}$ & $\begin{array}{r}0 \\
998\end{array}$ & $\overline{\text { Elec. }}$ & $\overline{985}$ & -- & -- & $\begin{array}{l}\text { Sentry } 204 \text {; replaced on } 7 / 12 / 79 \text { with } \\
\text { sentry } 494 \text {. }\end{array}$ \\
\hline \multirow[t]{3}{*}{$S B-07-41-34-C$} & CEP & $4 / 09 / 79$ & $6 / 13 / 79$ & 0 & -- & -- & $\begin{array}{l}\text { Engine } \\
\text { hours }\end{array}$ & 0 & \multirow[t]{3}{*}{ Sentry 248} \\
\hline & & $\begin{array}{l}6 / 13 / 79 \\
8 / 25 / 79\end{array}$ & $\begin{array}{l}8 / 25 / 79 \\
9 / 19 / 79\end{array}$ & $\begin{array}{l}416 \\
124\end{array}$ & -- & -- & -- & -- & \\
\hline & & & Total-- & 540 & & & & & \\
\hline \multirow[t]{2}{*}{$S B-08-36-31-B$} & $\mathrm{CEP}$ & $\begin{array}{l}4 / 16 / 79 \\
6 / 14 / 79\end{array}$ & $\begin{array}{l}6 / 14 / 79 \\
9 / 19 / 79\end{array}$ & $\begin{array}{l}150 \\
510 \\
\end{array}$ & -- & - & $\cdots$ & $\cdots$ & \multirow[t]{2}{*}{ Sentry 226.} \\
\hline & & & Tota1--- & 660 & & & & & \\
\hline \multirow[t]{2}{*}{$\mathrm{SB}-08-38-06-\mathrm{AA}$} & $\mathrm{CEP}$ & $\begin{array}{l}4 / 16 / 79 \\
8 / 27 / 79\end{array}$ & $\begin{array}{l}8 / 27 / 79 \\
9 / 19 / 79\end{array}$ & $\begin{array}{l}392 \\
369 \\
\end{array}$ & - & -- & -- & -- & \multirow[t]{2}{*}{ Sentry 200.} \\
\hline & & & Tota1--- & 761 & & & & & \\
\hline \multirow[t]{2}{*}{$S B-08-38-34-A D$} & CEP & $4 / 16 / 79$ & $9 / 19 / 79$ & 950 & -- & -- & $\begin{array}{l}\text { Engine } \\
\text { hours }\end{array}$ & 814 & \multirow[t]{2}{*}{ Sentry 229} \\
\hline & & & Total--- & 950 & & & Total & --814 & \\
\hline \multirow[t]{2}{*}{$S B-08-39-07-B$} & CEP & $4 / 16 / 79$ & $9 / 19 / 79$ & 960 & Elec. & 952 & -- & -- & \multirow[t]{2}{*}{ Sentry 220} \\
\hline & & & Total--- & 960 & & 952 & & & \\
\hline $\begin{array}{l}\text { SB-08-41-14-CA } \\
\text { SB-08-41-14-CC }\end{array}$ & JLP & $\begin{array}{l}4 / 30 / 79 \\
7 / 19 / 79 \\
8 / 18 / 79\end{array}$ & $\begin{array}{c}7 / 19 / 79 \\
8 / 18 / 79 \\
10 / 22 / 79 \\
\text { Total- }\end{array}$ & $\begin{array}{r}316 \\
649 \\
726 \\
1,691\end{array}$ & Elec. & $\overline{665}$ & -- & $\overline{---}$ & $\begin{array}{l}\text { Sentry } 189 \text { measured time on well } \\
\text { SB-08-41-14-CA only; no time data } \\
\text { collected on well SB-08-41-14-CC. }\end{array}$ \\
\hline
\end{tabular}


Table 12a.--Pumpage data for irrigation sites in Dundy County, Nebraska

\begin{tabular}{|c|c|c|c|c|c|c|c|c|c|c|c|c|}
\hline \multirow{2}{*}{$\begin{array}{l}\text { Site } \\
\text { location }\end{array}$} & \multirow{2}{*}{$\begin{array}{l}\text { Station } \\
\text { identifier }\end{array}$} & \multicolumn{4}{|c|}{ Irrigated crop information } & \multicolumn{6}{|c|}{ Well information } & \multirow[b]{2}{*}{ Remarks } \\
\hline & & Type & System & Acres & Source & $\begin{array}{l}\text { Depth } \\
(\mathrm{ft})\end{array}$ & $\begin{array}{r}\text { Water } \\
\text { level } \\
(\mathrm{ft})\end{array}$ & $\begin{array}{l}\text { Diam- } \\
\text { meter } \\
\text { (in) }\end{array}$ & $\begin{array}{l}\text { Pump } \\
\text { hp. }\end{array}$ & $\begin{array}{l}\text { Energy } \\
\text { source }\end{array}$ & $\begin{array}{l}\text { Energy } \\
\text { rating }\end{array}$ & \\
\hline$S B-01-40-22-C A$ & 400214101493700 & $\begin{array}{l}\text { Milo } \\
\text { Alfalfa }\end{array}$ & $\begin{array}{l}\text { Pivot } \\
\text { Pivot }\end{array}$ & $\begin{array}{l}70 \\
70\end{array}$ & $\begin{array}{l}\text { Meas. } \\
\text { Meas. }\end{array}$ & -- & --- & -- & --- & Elec. & --- & \\
\hline$S B-01-40-26-B B$ & 400148101484601 & Alfalfa & Pivot & 97 & Rept. & -- & -- & -- & -- & Elec. & -- & \\
\hline $\mathrm{SB}-02-38-34-\mathrm{AC}$ & 400555101354301 & Corn & $\begin{array}{l}\text { Sprink- } \\
\quad \text { ler }\end{array}$ & 65 & Rept. & --- & -- & -- & -- & Diese1 & -- & \\
\hline$S B-03-37-34-C B$ & $401 j 53101292601$ & Corn & Pivot & 140 & Rept. & 210 & 86 & 15.5 & -- & Elec. & -- & Water-1evel measurement on $1 / 11 / 71$. \\
\hline$S B-03-38-17-B A$ & 401356101381801 & Alfalfa & $\begin{array}{l}\text { Sprink- } \\
\text { ler }\end{array}$ & 80 & Rept. & 160 & 33 & 16 & --- & -- & -- & Water-level measurement on $6 / 11 / 74$. \\
\hline$S B-03-38-29-A C$ & 401159101380011 & Corn & Pivot & 127 & Rept. & 260 & -- & $-\cdots$ & -- & Elec. & -- & \\
\hline$S B-03-39-18-C D$ & 401325101462101 & Corn & Pivot & 146 & Meas. & -- & -- & -- & -- & Diesel & -- & \\
\hline$S B-03-39-30-D$ & 401141101454701 & Corn & Pivot & 130 & Rept. & 280 & -- & -- & -- & Diesel & -- & \\
\hline
\end{tabular}


Table 12b.--Pumpage data for irrigation sites in Dundy County, Nebraska

\begin{tabular}{|c|c|c|c|c|c|}
\hline \multirow{2}{*}{$\begin{array}{l}\text { Site } \\
\text { location }\end{array}$} & \multirow{2}{*}{$\begin{array}{l}\text { Field } \\
\text { person }\end{array}$} & \multicolumn{4}{|c|}{ Discharge information } \\
\hline & & Date & $\begin{array}{l}\text { Discharge } \\
\text { (gal/min). }\end{array}$ & Method & Remarks \\
\hline$S B-01-40-22-C A$ & CEP & $6 / 19 / 79$ & 615 & T.T. & Measurement on 8-in steel tubing. \\
\hline$S B-01-40-26-B 3$ & CEP & $6 / 19 / 79$ & 210 & T.T. & Measurement on 8-in aluminum pipe. \\
\hline$S B-02-38-34-A C$ & JLP & $\begin{array}{l}7 / 20 / 79 \\
7 / 20 / 79 \\
7 / 20 / 79\end{array}$ & $\begin{array}{l}500 \\
250 \\
550\end{array}$ & $\begin{array}{l}\text { Dop. } \\
\text { Dop. } \\
\text { Inl. }\end{array}$ & $\begin{array}{l}\text { Measurement on } 6-\text { in aluminum pipe. } \\
\text { Polysonics. }\end{array}$ \\
\hline$S B-03-37-34-C B$ & JLP & $\begin{array}{l}7 / 17 / 79 \\
7 / 17 / 79\end{array}$ & $\begin{array}{l}735 \\
120\end{array}$ & $\begin{array}{l}\text { Dop. } \\
\text { Dop. }\end{array}$ & $\begin{array}{l}\text { Measurement on } 6 \text {-in steel tubing. } \\
\text { Polysonics. }\end{array}$ \\
\hline$S B-03-38-17-B A$ & JLP & $\begin{array}{l}7 / 24 / 79 \\
7 / 24 / 79 \\
7 / 24 / 79\end{array}$ & $\begin{array}{l}305 \\
3100 \\
700\end{array}$ & $\begin{array}{l}\text { Dop. } \\
\text { Dop. } \\
\text { Inl. }\end{array}$ & $\begin{array}{l}\text { Measurement on } 6 \text {-in aluminum pipe. } \\
\text { Polysonics. }\end{array}$ \\
\hline$S B-03-38-29-A C$ & JLP & $\begin{array}{l}7 / 17 / 79 \\
7 / 17 / 79 \\
7 / 17 / 79\end{array}$ & $\begin{array}{r}685 \\
1,000 \\
670\end{array}$ & $\begin{array}{l}\text { T.T. } \\
\text { Inl. } \\
\text { Dop. }\end{array}$ & Measurement on 8 -in steel tubing. \\
\hline$S B-03-39-18-C D$ & CEP & $\begin{array}{l}6 / 12 / 79 \\
6 / 12 / 79\end{array}$ & $\begin{array}{l}560 \\
700\end{array}$ & $\begin{array}{l}\text { T.T. } \\
\text { Inl. }\end{array}$ & $\begin{array}{l}\text { Clampitron had intermittant fault problems. } \\
\text { Measurement on } 8 \text {-in steel tubing. }\end{array}$ \\
\hline SB-03-39-30-D & CEP & $6 / 12 / 79$ & $\begin{array}{r}520 \\
* 820\end{array}$ & $\begin{array}{l}\text { T.T. } \\
\text { In1. }\end{array}$ & $\begin{array}{l}\text { Measurement on } 8 \text {-in steel tubing. } \\
\text { Reported season average from In-line flowmete }\end{array}$ \\
\hline
\end{tabular}


Table 12c.--Pumpage data for irrigation sites in Dundy County, Nebraska

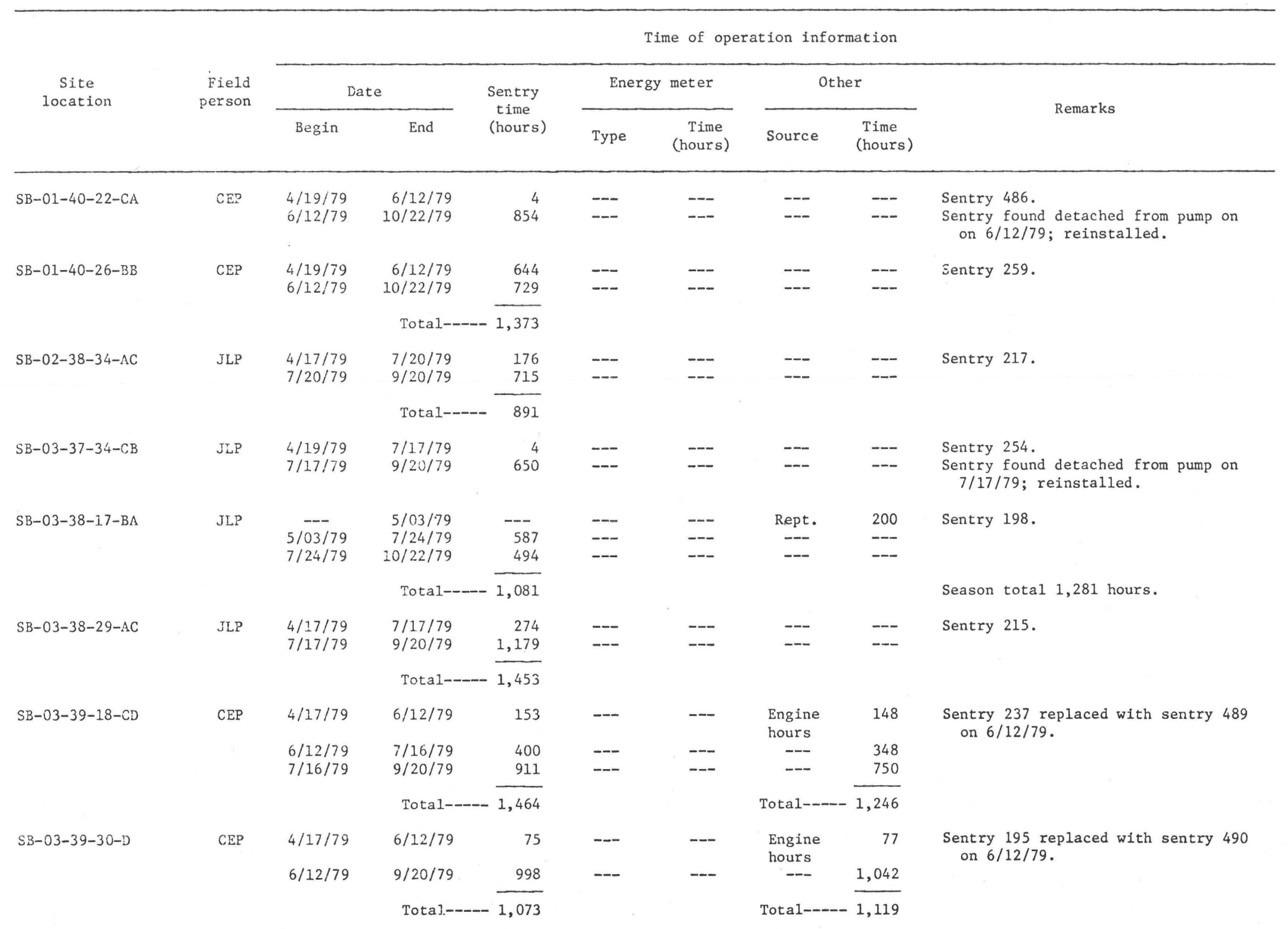


Table 12a.--Pumpage data for irrigation sites in Dundy County, Nebraska--Continued

\begin{tabular}{|c|c|c|c|c|c|c|c|c|c|c|c|c|}
\hline \multirow{2}{*}{$\begin{array}{c}\text { Site } \\
\text { location }\end{array}$} & \multirow{2}{*}{$\begin{array}{l}\text { Station } \\
\text { identifier }\end{array}$} & \multicolumn{4}{|c|}{ Irrigated crop information } & \multicolumn{6}{|c|}{ Well information } & \multirow[b]{2}{*}{ Remarks } \\
\hline & & Type & System & Acres & Source & $\begin{array}{r}\text { Depth } \\
(\mathrm{ft})\end{array}$ & $\begin{array}{r}\text { Water } \\
\text { level } \\
(\mathrm{ft})\end{array}$ & $\begin{array}{l}\text { Diam- } \\
\text { meter } \\
\text { (in) }\end{array}$ & $\begin{array}{l}\text { Pump } \\
\text { hp. }\end{array}$ & $\begin{array}{l}\text { Energy } \\
\text { source }\end{array}$ & $\begin{array}{l}\text { Energy } \\
\text { rating }\end{array}$ & \\
\hline $\mathrm{SB}-04-39-31-\mathrm{DD}$ & 401600101454701 & Corn & Pivot & 124 & Rept. & 300 & -- & 16 & -- & --- & -- & \\
\hline $\mathrm{SB}-04-40-32-\mathrm{CA}$ & 401607101515301 & Corn & Pivot & 136 & Meas. & 330 & -- & 16 & -- & Elec. & 61.5 & \\
\hline $\mathrm{SP}-04-41-31-\mathrm{CD}$ & 401555101595411 & Corn & Pivot & 160 & Rept. & 257 & 0.65 & -- & -- & Elec. & -- & Water-level measurement on $5 / 27 / 65$ \\
\hline
\end{tabular}

官 
Table 12b.--Pumpage áata for irmigation sites in Dundy County, Nebraska--Continued

\begin{tabular}{|c|c|c|c|c|c|}
\hline \multirow{2}{*}{$\begin{array}{c}\text { Site } \\
\text { location }\end{array}$} & \multirow{2}{*}{$\begin{array}{r}\text { Field } \\
\text { person }\end{array}$} & \multicolumn{4}{|c|}{ Discharge information } \\
\hline & & Date & $\begin{array}{l}\text { Discharge } \\
\text { (gal/min) }\end{array}$ & Method & Remarks \\
\hline$S B-04-39-31-D D$ & CEP & $7 / 25 / 79$ & 750 & Inl. & Measurement on 8 -in steel tubing. \\
\hline $\mathrm{SB}-04-40-32-\mathrm{CA}$ & CEP & $\begin{array}{l}7 / 16 / 79 \\
7 / 16 / 79\end{array}$ & $\begin{array}{l}805 \\
790\end{array}$ & $\begin{array}{l}\text { T. T. } \\
\text { In1. }\end{array}$ & Measurement on 8 -in steel tubing. \\
\hline $\mathrm{SB}-04-41-31-\mathrm{CD}$ & CEP & $\begin{array}{l}6 / 14 / 79 \\
6 / 14 / 79\end{array}$ & $\begin{array}{l}1,030 \\
1,035\end{array}$ & $\begin{array}{l}\text { T. T. } \\
\text { Inl. }\end{array}$ & Measurement on 8 -in steel tubing. \\
\hline
\end{tabular}


Table 12c.--Pumpage data for irrigation sites in Dundy County, Nebraska--Continued

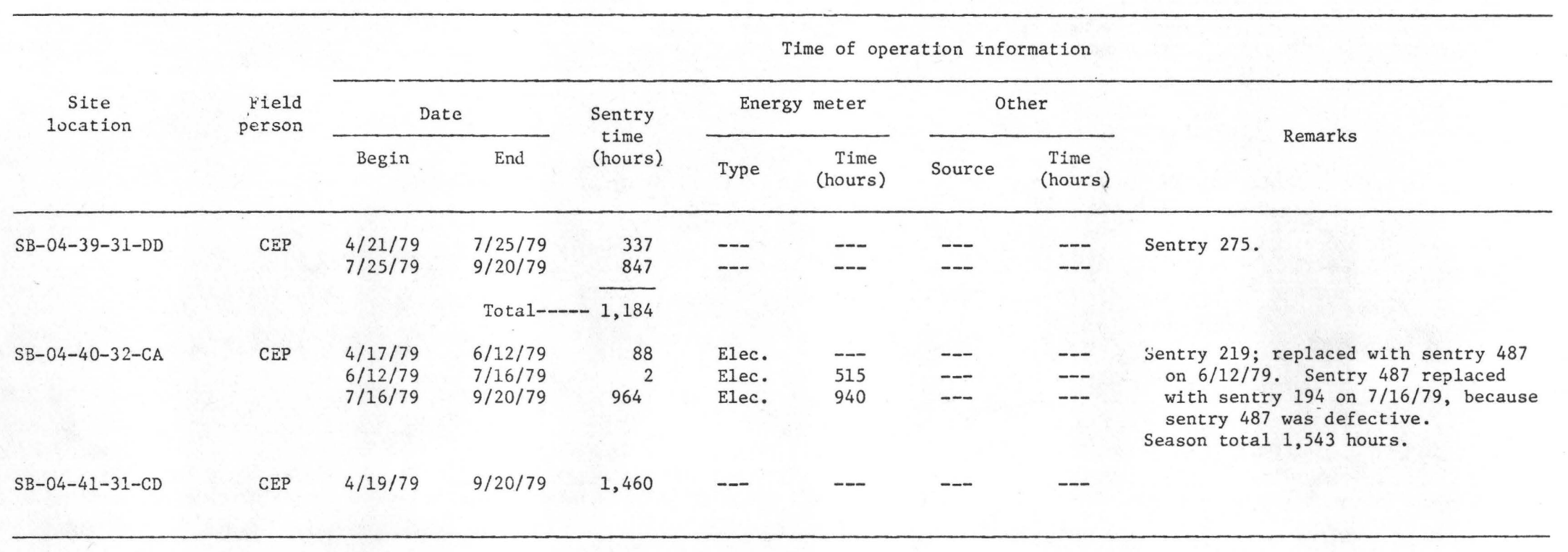

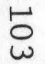


Table 13a.--Pumpage data for irrigation sites in Perkins County, Nebraska

\begin{tabular}{|c|c|c|c|c|c|c|c|c|c|c|c|c|}
\hline \multirow{2}{*}{$\begin{array}{c}\text { Site } \\
\text { location }\end{array}$} & \multirow{2}{*}{$\begin{array}{l}\text { Station } \\
\text { identifier }\end{array}$} & \multicolumn{4}{|c|}{ Irrigated crop information } & \multicolumn{6}{|c|}{ Well information } & \multirow{2}{*}{ Remarks } \\
\hline & & Type & System & Acres & Source & $\begin{array}{l}\text { Depth } \\
(\mathrm{ft})\end{array}$ & $\begin{array}{r}\text { Water } \\
\text { level } \\
\text { (ft) }\end{array}$ & $\begin{array}{l}\text { Diam- } \\
\text { meter } \\
\text { (in) }\end{array}$ & $\begin{array}{c}\text { Pump } \\
\text { hp. }\end{array}$ & $\begin{array}{l}\text { Energy } \\
\text { source }\end{array}$ & $\begin{array}{l}\text { Energy } \\
\text { rating }\end{array}$ & \\
\hline $\mathrm{SB}-09-35-22-\mathrm{CC}$ & 404337101181701 & Corn & 2 Pivots & 246 & Rept. & 363 & -- & 18 & -- & Elec. & -- & \\
\hline$S B-09-38-10-A A$ & 404610101380000 & Corn & Pivot & 130 & Rept. & 400 & -- & 16 & $-\cdots$ & E1ec. & -- & \\
\hline SB-09-38-17-CD & 404445101405801 & Corn & Pivot & 130 & Rept. & 280 & -- & 16 & -- & Elec. & -- & \\
\hline$S B-09-41-06-D C$ & 404621102022501 & Beets & Pivot & 145 & Rept. & -- & -- & -- & 100 & Elec. & 77.4 & \\
\hline $\mathrm{SB}-11-36-03-\mathrm{AB}$ & 405730101243801 & Corn & Pivot & 400 & Rept. & 363 & -- & 18 & 100 & Elec. & -- & \\
\hline$S B-11-36-29-B$ & 405353101272401 & Corn & Pivot & 1.00 & Rept. & 490 & -- & 16 & 100 & Elec. & $-\infty$ & \\
\hline SB-11-37-25-CC & 405321101294101 & Corn & Pivot & 130 & Rept. & 485 & -- & 16 & -- & Diesel & -- & \\
\hline $\begin{array}{l}\text { SB-12-38-19-D0 } \\
\text { SB-12-38-19-00 }\end{array}$ & $\begin{array}{l}405935101210101 \\
405948101211801\end{array}$ & Corn & Pivot & 250 & Rept. & 377 & -- & 16 & -- & Elec. & -- & 2 wells operate this one pivot. \\
\hline
\end{tabular}


Table 13b.--Pumpage data for irrigation sites in Perkins County, Nebraska

\begin{tabular}{|c|c|c|c|c|c|}
\hline \multirow{2}{*}{$\begin{array}{l}\text { Site } \\
\text { location }\end{array}$} & \multirow{2}{*}{$\begin{array}{r}\text { Field } \\
\text { person }\end{array}$} & \multicolumn{4}{|c|}{ Discharge information } \\
\hline & & Date & $\begin{array}{l}\text { Discharge } \\
\text { (gal/min) }\end{array}$ & Method & Remarks \\
\hline$S B-09-35-22-C C$ & JLP & $\begin{array}{l}8 / 07 / 79 \\
8 / 07 / 79\end{array}$ & $\begin{array}{l}770 \\
800\end{array}$ & $\begin{array}{l}\text { T.T. } \\
\text { In1. }\end{array}$ & $\begin{array}{l}\text { Measurement on } 8 \text {-in steel pipe and } 8 \text {-in aluminum pipe. } \\
\text { McCrometer inline flowmeter. }\end{array}$ \\
\hline$S B-09-38-10-A A$ & CEP & $\begin{array}{l}6 / 14 / 79 \\
6 / 14 / 79\end{array}$ & $\begin{array}{l}640 \\
930\end{array}$ & $\begin{array}{l}\text { T.T. } \\
\text { In1. }\end{array}$ & Measurement on 8 -in steel tubing. \\
\hline$S B-09-38-17-C D$ & CEP & $\begin{array}{l}8 / 07 / 79 \\
8 / 07 / 79\end{array}$ & $\begin{array}{l}620 \\
885\end{array}$ & $\begin{array}{l}\text { T.T. } \\
\text { Inl. }\end{array}$ & Measurement on 8-in steel tubing. \\
\hline SB-09-41-06-DC & JLP & $\begin{array}{l}7 / 24 / 79 \\
7 / 24 / 79\end{array}$ & $\begin{array}{l}775 \\
630\end{array}$ & $\begin{array}{l}\text { T.T. } \\
\text { Dop. }\end{array}$ & Measurement on 8-in steel tubing. \\
\hline $\mathrm{SB}-11-36-03-\mathrm{AB}$ & JLP & $\begin{array}{l}8 / 09 / 79 \\
8 / 09 / 79\end{array}$ & $\begin{array}{r}895 \\
1,640\end{array}$ & $\begin{array}{l}\text { T.T. } \\
\text { Dop. }\end{array}$ & Measurement on 8-in aluminum pipe. \\
\hline$S B-11-36-29-B$ & JLP & $8 / 07 / 79$ & 515 & T.T. & Measurement on 8 -in steel tubing. \\
\hline$S B-11-37-25-C C$ & CEP & $8 / 07 / 79$ & 650 & T.T. & Measurement on 8-in steel tubing. \\
\hline $\begin{array}{l}\text { SB-12-38-19-D0 } \\
\text { SB-12-38-19-00 }\end{array}$ & CEP & $8 / 07 / 79$ & 1,530 & T.T. & $\begin{array}{l}\text { Measurement on } 8 \text {-in steel tubing. } \\
\text { Combined discharge from both wells. }\end{array}$ \\
\hline
\end{tabular}


Table 13c.--Pumpage data for irrigation sites in Perkins County, Nebraska

\begin{tabular}{|c|c|c|c|c|c|c|c|c|c|}
\hline \multirow{3}{*}{$\begin{array}{c}\text { Site } \\
\text { location }\end{array}$} & \multirow{3}{*}{$\begin{array}{l}\text { Field } \\
\text { person }\end{array}$} & \multicolumn{8}{|c|}{ Time of operation information } \\
\hline & & \multicolumn{2}{|c|}{ Date } & \multirow{2}{*}{$\begin{array}{l}\text { Sentry } \\
\text { time } \\
\text { (hours) }\end{array}$} & \multicolumn{2}{|c|}{ Energy meter } & \multicolumn{2}{|c|}{ Other } & \multirow{2}{*}{ Remarks } \\
\hline & & Begin & End & & Type & $\begin{array}{c}\text { Time } \\
\text { (hours) }\end{array}$ & Source & $\begin{array}{l}\text { Time } \\
\text { (hours) }\end{array}$ & \\
\hline \multirow[t]{2}{*}{$S B-09-35-22-C C$} & JLP & $\begin{array}{l}4 / 19 / 79 \\
8 / 07 / 79\end{array}$ & $\begin{array}{l}8 / 07 / 79 \\
9 / 19 / 79\end{array}$ & $\begin{array}{l}498 \\
579\end{array}$ & -- & -- & $-\infty$ & -- & Sentry 233 \\
\hline & & & Tota1-- & $-1,077$ & & & & & \\
\hline \multirow[t]{2}{*}{$S B-09-38-10-A A$} & CEP & $\begin{array}{l}4 / 18 / 79 \\
6 / 14 / 79\end{array}$ & $\begin{array}{l}6 / 14 / 79 \\
9 / 19 / 79\end{array}$ & $\begin{array}{l}391 \\
357\end{array}$ & $\begin{array}{l}\text { Elec. } \\
\text { Elec. }\end{array}$ & $\begin{array}{l}419 \\
346\end{array}$ & $-\cdots$ & --- & Sentry 246. \\
\hline & & & Tota1-- & 748 & & 765 & & & \\
\hline \multirow[t]{2}{*}{$S B-09-38-17-C D$} & CEP & $\begin{array}{l}4 / 19 / 79 \\
8 / 07 / 79\end{array}$ & $\begin{array}{l}8 / 07 / 79 \\
9 / 20 / 79\end{array}$ & $\begin{array}{l}368 \\
362 \\
\end{array}$ & Elec. & $\overline{342}$ & -- & -- & Sentry 183. \\
\hline & & & Total-- & 730 & & & & & \\
\hline SB-09-41-06-DC & JLP & $\begin{array}{l}4 / 30 / 79 \\
7 / 24 / 79\end{array}$ & $\begin{array}{l}7 / 24 / 79 \\
9 / 20 / 79\end{array}$ & 409 & -- & $\overline{--}$ & $-\infty$ & -- & $\begin{array}{l}\text { Sentry } 255 . \\
\text { Sentry lost. }\end{array}$ \\
\hline \multirow[t]{2}{*}{$S B-I 1-36-03-A B$} & JLP & $\begin{array}{l}4 / 19 / 79 \\
8 / 09 / 79\end{array}$ & $\begin{array}{l}8 / 09 / 79 \\
9 / 19 / 79\end{array}$ & $\begin{array}{l}252 \\
469\end{array}$ & $\begin{array}{l}\text { Elec. } \\
-\end{array}$ & 249 & $-\infty$ & $\overline{--}$ & Sentry 203. \\
\hline & & & Total-- & 721 & & & & & \\
\hline$S B-11-36-29-B$ & JLP & $\begin{array}{l}4 / 19 / 79 \\
8 / 07 / 79\end{array}$ & $\begin{array}{l}8 / 07 / 79 \\
9 / 19 / 79\end{array}$ & $\begin{array}{r}1339 \\
75\end{array}$ & Elec. & $\overline{282}$ & -- & -- & $\begin{array}{l}\text { Season total } 1,621 \text { hours. } \\
\text { Sentry } 184 \text {. Sentry reading appears } \\
\text { low; use time from electric meter } \\
\text { for } 8 / 7 / 79-9 / 19 / 79 \text {. }\end{array}$ \\
\hline $\mathrm{SB}-11-37-25-\mathrm{CC}$ & CEP & $\begin{array}{l}4 / 18 / 79 \\
8 / 07 / 79\end{array}$ & $\begin{array}{l}8 / 07 / 79 \\
9 / 20 / 79\end{array}$ & 561 & $\cdots$ & -- & $\cdots$ & $-\cdots$ & $\begin{array}{l}\text { Sentry } 241 . \\
\text { Sentry lost. }\end{array}$ \\
\hline SB-12-38-19-DO & CEP & $\begin{array}{l}4 / 19 / 79 \\
8 / 07 / 79\end{array}$ & $\begin{array}{l}8 / 07 / 79 \\
9 / 19 / 79\end{array}$ & $\begin{array}{l}648 \\
602 \\
\end{array}$ & $-\infty$ & -- & $\cdots$ & -- & Sentry 191. \\
\hline SE-12-38-19-00 & CE? & $\begin{array}{l}4 / 19 / 79 \\
8 / 07 / 79\end{array}$ & $\begin{array}{l}\text { Total-- } \\
8 / 07 / 79 \\
9 / 19 / 79\end{array}$ & $\begin{array}{r}1,250 \\
676 \\
609\end{array}$ & $-\infty$ & $-\infty$ & -- & -- & Sentry 224. \\
\hline & & & Total-- & $-1,285$ & & & & & \\
\hline
\end{tabular}


Table 14a.--Pumpage data for irrigation sites in Hockley County, Texas

\begin{tabular}{|c|c|c|c|c|c|c|c|c|c|c|c|c|}
\hline \multirow{2}{*}{$\begin{array}{l}\text { Site } \\
\text { location }\end{array}$} & \multirow{2}{*}{$\begin{array}{l}\text { Station } \\
\text { identifier }\end{array}$} & \multicolumn{4}{|c|}{ Irrigated crop information } & \multicolumn{6}{|c|}{ Well information } & \multirow{2}{*}{ Remarks } \\
\hline & & Type & System & Acres & Source & $\begin{array}{l}\text { Depth } \\
(\mathrm{ft})\end{array}$ & $\begin{array}{r}\text { Water } \\
\text { level } \\
(f t)\end{array}$ & $\begin{array}{l}\text { Diam- } \\
\text { meter } \\
\text { (in) }\end{array}$ & $\begin{array}{l}\text { Pump } \\
\text { hp. }\end{array}$ & $\begin{array}{l}\text { Energy } \\
\text { source }\end{array}$ & $\begin{array}{l}\text { Energy } \\
\text { rating }\end{array}$ & \\
\hline $333721 \mathrm{~N} 1021628 \mathrm{~W}$ & 333721102162801 & Cotton & Plood & 80 & Meas. & 174 & 102 & 10 & 40 & Elec. & 27 & Four wells at site. \\
\hline $334026 \mathrm{~N} 1023541 \mathrm{~W}$ & 334025102354101 & Cotton & -- & 30 & Meas. & 190 & 137 & 16 & -- & N.Gas & -- & \\
\hline $334336 \mathrm{~N} 1021417 \mathrm{~W}$ & 334336102141701 & Cotton & Flood & 30 & Meas. & 158 & 107 & 16 & $15^{7.5}$ & Elec. & 19 & Two wells at site. \\
\hline $334201 \times 1023429 \mathrm{~W}$ & 334201102342901 & Cotton & Pivot & 540 & Meas. & 225 & 126 & 16 & -- & N.Gas & .721 & Three wells at site. \\
\hline $334313 \mathrm{~N} 1022434 \mathrm{~W}$ & 334313102243401 & Cotton & Sprinkler & 25 & Meas. & 134 & 93 & 14 & 20 & Elec. & -- & \\
\hline $334350 \mathrm{~N} 1021725 \mathrm{~W}$ & 334350102172501 & Cotton & -- & -- & Meas. & -- & 87 & 132 & 12 & Elec. & -- & Abandoned cotton crop. \\
\hline $333910 \mathrm{~N} 1022129 \mathrm{~W}$ & 333910102212901 & Cotton & Sprinkler & 10 & Meas. & 225 & 173 & 10 & -- & Elec. & -- & Cotton not watered. \\
\hline $333642 \mathrm{~N} 1020927 \mathrm{~W}$ & 333542102092701 & Cotton & Flood & 102 & Meas. & 144 & 124 & 12 & -- & Elec. & 650 & $\begin{array}{l}\text { Power rating for only } 3 \text { of } 4 \text { wells } \\
\text { in system. }\end{array}$ \\
\hline $332504 \mathrm{~N} 1021912 \mathrm{~W}$ & 332504102191201 & Cotton & Sprinkler & 293 & Meas. & 212 & 152 & 12 & $\begin{array}{ll}10 \quad 1 & \\
& 2\end{array}$ & $\begin{array}{l}1 \text { Elec. } \\
\text { and } \\
2 \text { N.Gas }\end{array}$ & $\cdots$ & \\
\hline $334051 \mathrm{~N} 1022112 \mathrm{~W}$ & 334051102211201 & Cotton & Sprinkler & 131 & Meas. & 150 & 101 & 14 & 15 & Elec. & 17 & Two wells at site. \\
\hline $333519 \mathrm{~N} 1020600 \mathrm{~W}$ & 333519102060001 & Cotton & Flood & 75 & Meas. & 150 & 99 & 14 & 30 & Elec. & -- & \\
\hline $333152 \mathrm{~N} 1022604 \mathrm{~W}$ & 333152102260401 & Cotton & Flood & 150 & Meas. & 212 & 159 & 16 & -- & N.Gas & -- & \\
\hline $333358 \mathrm{~N} 1021500 \mathrm{~W}$ & 333358102150001 & $\operatorname{Cot} n n$ & Flood & 35 & Meas. & 136 & 109 & 12 & 10 & Elec. & 5 & \\
\hline
\end{tabular}


Table 14b.--Prompage data for irrigation sites in Hockley County, Texas

\begin{tabular}{|c|c|c|c|c|c|}
\hline \multirow{2}{*}{$\begin{array}{c}\text { Site } \\
\text { location }\end{array}$} & \multirow{2}{*}{$\begin{array}{r}\text { Field } \\
\text { person }\end{array}$} & \multicolumn{4}{|c|}{ Discharge information } \\
\hline & & Date & $\begin{array}{l}\text { Discharge } \\
\text { (gal/min) }\end{array}$ & Method & Remarks \\
\hline $333721 \mathrm{~N} 1021628 \mathrm{~N}$ & PLR & $4 / 05 / 79$ & $\begin{array}{l}250 \\
210\end{array}$ & $\begin{array}{l}\text { Buck. } \\
\text { Dop. }\end{array}$ & Yield of four wells. \\
\hline $334026 \mathrm{~N} 1023541 \mathrm{~N}$ & -- & -- & -- & -- & No discharge measurements were made in 1979. \\
\hline $334336 \mathrm{~N} 1021417 \mathrm{~W}$ & $\begin{array}{l}\text { PLR } \\
\text { WAW }\end{array}$ & $4 / 28 / 79$ & $\begin{array}{l}140 \\
140\end{array}$ & $\begin{array}{l}\text { Buck. } \\
\text { Dop. }\end{array}$ & \\
\hline $334201 \mathrm{~N} 1023429 \mathrm{~W}$ & $\begin{array}{l}\text { WAW } \\
\text { PLR }\end{array}$ & $8 / 08 / 79$ & 1,490 & T.T. & \\
\hline $334313 \mathrm{~N} 1022434 \mathrm{~W}$ & -- & -- & -- & -- & No discharge measurements were made in 1979. \\
\hline $334350 \mathrm{~N} 1021725 \mathrm{~W}$ & -- & -- & -- & -- & No discharge measurements were made in 1979. \\
\hline 333910N1022129W & -- & -- & -- & -- & No discharge measurements were made in 1979. \\
\hline $333642 \mathrm{~N} 1020927 \mathrm{w}$ & PLR & $4 / 19 / 79$ & $\begin{array}{r}80 \\
230\end{array}$ & $\begin{array}{l}\text { Buck. } \\
\text { Dop. }\end{array}$ & $\begin{array}{l}\text { Three of four wells. } \\
\text { Three of four wells. }\end{array}$ \\
\hline $332504 \mathrm{~N} 1021912 \mathrm{~W}$ & -- & -- & -- & -- & No discharge measurements were made in 1979. \\
\hline $334051 N 1022112 \mathrm{~W}$ & WAW & $4 / 25 / 79$ & 140 & Other & Hand held Pres gage. \\
\hline $333519 \mathrm{~N} 1020600 \mathrm{~W}$ & -- & -- & -- & --- & No discharge measurements were made in 1979. \\
\hline $333152 \mathrm{~N} 1022604 \mathrm{~N}$ & WAW & $4 / 28 / 79$ & 530 & Buck. & \\
\hline $333358 \mathrm{N1021500 \textrm {N }}$ & $\begin{array}{l}\text { WAW } \\
\text { PLR }\end{array}$ & $\begin{array}{l}4 / 14 / 79 \\
4 / 19 / 79\end{array}$ & $\begin{array}{l}50 \\
50\end{array}$ & $\begin{array}{l}\text { Buck. } \\
\text { Buck. }\end{array}$ & \\
\hline
\end{tabular}


Table 14c.--Pumpage data for irrigation sites in Hockley County, Texas

\begin{tabular}{|c|c|c|c|c|c|c|c|c|c|}
\hline \multirow{3}{*}{$\begin{array}{l}\text { Site } \\
\text { location }\end{array}$} & \multirow{3}{*}{$\begin{array}{r}\text { Field } \\
\text { person }\end{array}$} & \multicolumn{8}{|c|}{ Time of operation information } \\
\hline & & \multicolumn{2}{|c|}{ Date } & \multirow{2}{*}{$\begin{array}{l}\text { Sentry } \\
\text { time } \\
\text { (hours) }\end{array}$} & \multicolumn{2}{|c|}{ Energy meter } & \multicolumn{2}{|c|}{ Other } & \multirow{2}{*}{ Remarks } \\
\hline & & Begin & End & & Type & $\begin{array}{c}\text { Time } \\
\text { (hours) }\end{array}$ & Source & $\begin{array}{l}\text { Time } \\
\text { (hours) }\end{array}$ & \\
\hline $333721 \times 1021628 \mathrm{~W}$ & PLR & $4 / 04 / 79$ & $8 / 08 / 79$ & 420 & Elec. & 425 & -- & -- & $\begin{array}{l}\text { Sentry } 035 . \\
\text { Sentry distorted. }\end{array}$ \\
\hline $334026 \times 1023541 W$ & WAW & - & $8 / 20 / 79$ & 6 & N.Gas & 6 & - & - & Sentry 046 \\
\hline $334336 \mathrm{~N} 1021417 \mathrm{~W}$ & $\begin{array}{l}\text { PLR } \\
\text { WAW }\end{array}$ & $4 / 03 / 79$ & $8 / 22 / 79$ & 720 & Elec. & 700 & - & $-\infty$ & Sentry 056 \\
\hline $334201 \mathrm{~N} 1023429 \mathrm{~W}$ & $\begin{array}{l}\text { WAN } \\
\text { PLR }\end{array}$ & $4 / 14 / 79$ & $8 / 20 / 79$ & 574 & N.Gas & 536 & $-\cdots$ & - & Sentry 034 \\
\hline $334313 \mathrm{~N} 1022434 \mathrm{~W}$ & PLR & -- & $8 / 20 / 79$ & 1 & Elec. & 0 & - & -- & Sentry 068 \\
\hline $334350 \mathrm{~N} 1021725 \mathrm{~W}$ & PLR & - & -- & -- & Elec. & - & $-\infty$ & - & Sentry not installed in 1979. \\
\hline $333910 \mathrm{~N} 1022129 \mathrm{~W}$ & PLR & $\cdots$ & -- & -- & Elec. & -- & $-\cdots$ & $-\infty$ & $\begin{array}{l}\text { Sentry not installed in } 1979 . \\
\text { Subm. pump covered with sand. }\end{array}$ \\
\hline $333642 N 1020927 W$ & PLR & $4 / 05 / 79$ & $8 / 21 / 79$ & 540 & Elec. & 502 & $\cdots$ & - & Sentry 059 \\
\hline $332504 \mathrm{~N} 1021912 \mathrm{~W}$ & WAW & $\begin{array}{l}4 / 03 / 79 \\
7 / 13 / 79\end{array}$ & $\begin{array}{l}7 / 13 / 79 \\
8 / 08 / 79\end{array}$ & $\begin{array}{r}98 \\
1\end{array}$ & $\begin{array}{l}\text { Elec. } \\
\text { Elec. }\end{array}$ & $\begin{array}{r}145 \\
0\end{array}$ & - & $\ldots$ & $\begin{array}{l}\text { Sentry } 077 \text {. Sentry distorted. } \\
\text { Sentry } 327 \text {. Energy meter time } \\
\text { computed from estimated rating } \\
\text { factor. }\end{array}$ \\
\hline $334051 \mathrm{~N} 1022112 \mathrm{~W}$ & WAW & $5 / 07 / 79$ & $8 / 21 / 79$ & 32 & Elec. & 0 & -- & $-\infty$ & $\begin{array}{l}\text { Sentry } 332 \text {. Sentry installed after } \\
\text { irrigation season began. Used } \\
13,100 \mathrm{kWH} \text { of electricity early } \\
\text { in season. }\end{array}$ \\
\hline $333519 \mathrm{~N} 1020600 \mathrm{~W}$ & WAW & -- & $8 / 21 / 79$ & 5 & Elec. & 0 & -- & - & $\begin{array}{l}\text { Sentry } 337 . \text { Used } 4560 \mathrm{KWH} \text { of } \\
\text { electricity early in season. }\end{array}$ \\
\hline $333152 \mathrm{~N} 1022604 \mathrm{~W}$ & WAW & $4 / 03 / 79$ & $4 / 21 / 79$ & 370 & - & - & - & - & $\begin{array}{l}\text { Sentry } 078 \text {. } \\
\text { Field visits show } 370 \text { hours to be } \\
\text { reasonable. }\end{array}$ \\
\hline $333358 \mathrm{~N} 1 \mathrm{C} 215 \mathrm{COW}$ & $\begin{array}{l}\text { WAW } \\
\text { PLR }\end{array}$ & $4 / 14 / 79$ & $4 / 21 / 79$ & 408 & $\begin{array}{l}\text { Elec. } \\
\text { Elec. }\end{array}$ & 377 & $-\cdots$ & $\ldots$ & $\begin{array}{l}\text { Sentry } 065 \text {. } \\
\text { Sentry distorted. }\end{array}$ \\
\hline
\end{tabular}


Table 14a.--Pumpage data for irrigation sites in Hockley County, Texas--Continued

\begin{tabular}{|c|c|c|c|c|c|c|c|c|c|c|c|c|}
\hline \multirow{2}{*}{$\begin{array}{l}\text { Site } \\
\text { location }\end{array}$} & \multirow{2}{*}{$\begin{array}{l}\text { Station } \\
\text { identifier }\end{array}$} & \multicolumn{4}{|c|}{ Irrigated crop information } & \multicolumn{6}{|c|}{ Well information } & \multirow[b]{2}{*}{ Remarks } \\
\hline & & Type & System A & Acres & Source & $\begin{array}{l}\text { Depth } \\
\quad(f t)\end{array}$ & $\begin{array}{l}\text { Water } \\
\text { level } \\
(\mathrm{ft})\end{array}$ & $\begin{array}{l}\text { Diam- } \\
\text { meter } \\
\text { (in) }\end{array}$ & $\begin{array}{l}\text { Pump } \\
\text { hp. }\end{array}$ & $\begin{array}{l}\text { Energy } \\
\text { source }\end{array}$ & $\begin{array}{l}\text { Energy } \\
\text { rating }\end{array}$ & \\
\hline $333026 \mathrm{~N} 1021422 \mathrm{~W}$ & 333026102142201 & Cotton & -- & - & Meas. & 239 & 159 & 16 & -- & N.Gas & -- & Cotton crop abandoned. \\
\hline $333853 \mathrm{~N} 1023102 \mathrm{~W}$ & 333853102310201 & Cotton & Sprinkler & 88 & Meas. & 213 & 146 & 16 & 30. & N.Gas & -- & 25 hp. Booster used at site. \\
\hline $334412 \mathrm{~N} 1021920 \mathrm{~W}$ & 334412102192001 & Cotton & Sprinkler & 40 & Meas. & 117 & 74 & 10 & $\begin{array}{l}5 \\
5\end{array}$ & Elec. & -- & $\begin{array}{l}\text { Cotton not irrigated in } 1979 . \\
\text { Pumped for garden. } \\
\text { Two wells at site. }\end{array}$ \\
\hline $332916 \mathrm{~N} 1022216 \mathrm{~W}$ & 332916102221601 & Cotton & -- & 93 & Meas. & 207 & 158 & 16 & -- & Elec. & -- & Well not used in 1979. \\
\hline $332942 \mathrm{~N} 1021922 \mathrm{~N}$ & 332942102192201 & Cutton & Flood & 82 & Meas. & 238 & 153 & 14 & 30 & Elec. & 20 & \\
\hline $334007 \mathrm{~N} 1023341 \mathrm{~W}$ & 334007102334101 & Cotton & -- & 140 & Meas. & 250 & 144 & 16 & -- & N.Gas & -- & Well not used in 1979. \\
\hline $332915 \mathrm{~N} 1021328 \mathrm{~W}$ & 332915102132801 & Cotton & -- & -- & Meas. & 218 & 152 & 16 & 15 & Elec. & -- & Cotton crop abandoned. \\
\hline $334338 \mathrm{~N} 1021125 \mathrm{~W}$ & 334338102112501 & Cotton & Sprinkler & 52 & Meas. & 164 & 113 & 12 & -- & Elec. & & \\
\hline $333722 \mathrm{~N} 1022522 \mathrm{~W}$ & 333722102252201 & Cotton & Flood & 94 & Meas. & 265 & 165 & 16 & -- & N.Gas & .065 & Two wells at site. \\
\hline $333552 \mathrm{~N} 1021948 \mathrm{~W}$ & 333552102194801 & Cotton & $-\infty$ & 31 & Meas. & 200 & 139 & 16 & -- & N.Gas & -- & Well not used in 1979. \\
\hline $332636 \mathrm{~N} 1021006 \mathrm{~W}$ & 332636102100601 & Cotton & Sprinkler & 10 & Meas. & 150 & 192 & 9 & -- & Elec. & -- & \\
\hline $333157 \mathrm{~N} 1021751 \mathrm{~W}$ & 333157102175101 & Cotton & Flood & 76 & Meas. & 215 & 138 & 12 & $\begin{array}{l}25 \\
15\end{array}$ & Elec. & 29 & Two wells at site. \\
\hline $333715 \mathrm{~N} 1022054 \mathrm{~N}$ & 333715102205401 & Cotton & Sprinkler & 181 & Meas. & 207 & 147 & 13 & -- & N.Gas & .368 & Three wells at site. \\
\hline $334433 \mathrm{~N} 1021256 \mathrm{~W}$ & 334433102125601 & Cotton & Flood & 110 & Meas. & 160 & 107 & 12 & $\begin{array}{l}7.5+ \\
2+ \\
5\end{array}$ & Elec. & 13 & Three welis at site. \\
\hline
\end{tabular}


Table 14b.--Pumpage data for irrigation sites in Hockley County, Texas--Conti-ued

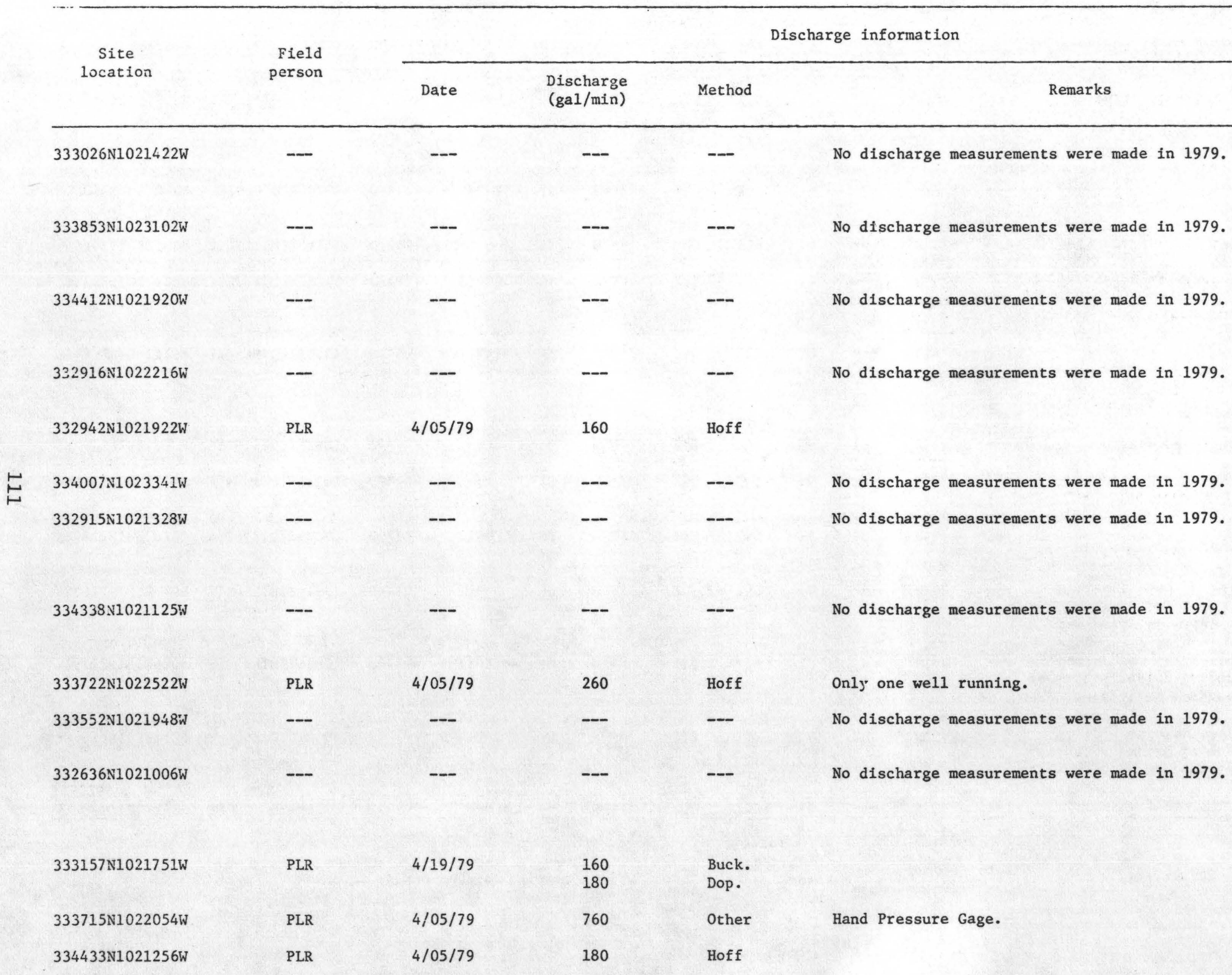


Table 14c.--Pumpage data for irrigation sites in Hockley County, Texas--Continued

\begin{tabular}{|c|c|c|c|c|c|c|c|c|c|}
\hline \multirow{3}{*}{$\begin{array}{l}\text { Site } \\
\text { location }\end{array}$} & \multirow{3}{*}{$\begin{array}{r}\text { Field } \\
\text { person }\end{array}$} & \multicolumn{8}{|c|}{ Time of operation information } \\
\hline & & \multicolumn{2}{|c|}{ Date } & \multirow{2}{*}{$\begin{array}{l}\text { Sentry } \\
\text { time } \\
\text { (hours) }\end{array}$} & \multicolumn{2}{|c|}{ Energy meter } & \multicolumn{2}{|c|}{ Other } & \multirow{2}{*}{ Remarks } \\
\hline & & Begin & End & & Type & $\begin{array}{c}\text { Time } \\
\text { (hours) }\end{array}$ & Source & $\begin{array}{l}\text { Time } \\
\text { (hours) }\end{array}$ & \\
\hline $333026 \mathrm{~N} 1021422 \mathrm{~W}$ & WAW & $4 / 25 / 79$ & $8 / 08 / 79$ & 281 & N.Gas & 294 & -- & -- & $\begin{array}{l}\text { Sentry } 338 . \\
\text { Energy meter time computed from } \\
\text { estimated rating factor. }\end{array}$ \\
\hline $333853 \mathrm{~N} 1023102 \mathrm{~W}$ & WAW & $4 / 03 / 79$ & $8 / 20 / 79$ & 54 & N.Gas & 40 & -- & $-\cdots$ & $\begin{array}{l}\text { Sentry } 348 . \\
\text { Energy meter time computed from } \\
\text { estimated rating factor. }\end{array}$ \\
\hline $334412 \mathrm{~N} 1021.920 \mathrm{~W}$ & WAW & -- & $8 / 21 / 79$ & 33 & Elec. & 78 & -- & -- & $\begin{array}{l}\text { Jentry } 014 . \\
\text { Energy meter time computed from } \\
\text { estimated rating factor. }\end{array}$ \\
\hline $332916 \mathrm{~N} 1022216 \mathrm{~W}$ & WAW & $5 / 10 / 79$ & $8 / 13 / 79$ & 1 & Elec. & 0 & $-\infty$ & -- & $\begin{array}{l}\text { Sentry 294. Electric meter never } \\
\text { installed. Sentry distorted. }\end{array}$ \\
\hline $332942 \mathrm{~N} 1021922 \mathrm{~W}$ & $\operatorname{PLR}$ & $4 / 05 / 79$ & $8 / 21 / 79$ & 286 & Elec. & 576 & -- & -- & $\begin{array}{l}\text { Sentry } 036 . \\
\text { Timer installed after season started. }\end{array}$ \\
\hline $334007 \mathrm{~N} 1023341 \mathrm{~W}$ & WAW & $5 / 07 / 79$ & $8 / 22 / 79$ & 6 & N.Gas & 0 & -- & -- & Sentry 334. \\
\hline $332915 \mathrm{~N} 1021328 \mathrm{~W}$ & WAN & $4 / 17 / 79$ & $7 / 13 / 79$ & 1,055 & Elec. & 560 & ---1 & -- & $\begin{array}{l}\text { Sentry } 041 . \\
\text { Sentry distorted. Energy meter } \\
\text { time computed using estimated } \\
\text { rating factor. }\end{array}$ \\
\hline $334338 \mathrm{~N} 1021125 \mathrm{~W}$ & WAW & $4 / 05 / 79$ & $8 / 20 / 79$ & 5 & Elec. & 0 & -- & --- & $\begin{array}{l}\text { Sentry } 005 \text {. } \\
490 \mathrm{KWH} \text { used before timer } \\
\text { installed. }\end{array}$ \\
\hline $333722 \mathrm{~N} 1022522 \mathrm{~W}$ & PLR & $4 / 03 / 79$ & $8 / 20 / 79$ & 306 & N.Gas & 354 & -- & --- & Sentry 069. \\
\hline 333552N1021948W & WAW & -- & $8 / 21 / 79$ & 1 & N.Gas & -- & --- & 0 & $\begin{array}{l}\text { Sentry } 047 \\
\text { Sentry distorted. }\end{array}$ \\
\hline $332636 \mathrm{~N} 1021006 \mathrm{~W}$ & WAW & $4 / 03 / 79$ & $8 / 13 / 79$ & 682 & Elec. & 594 & -- & -- & $\begin{array}{l}\text { Sentry } 071 \text {. } \\
\text { Sentry distorted. Energy meter } \\
\text { time computed using estimated } \\
\text { rating factor. }\end{array}$ \\
\hline $333157 \mathrm{~N} 1021751 \mathrm{~W}$ & PLK & $4 / 19 / 79$ & $8 / 21 / 79$ & 441 & Elec. & 423 & -- & -- & Sentry 322. \\
\hline $333715 \mathrm{~N} 1022054 \mathrm{~W}$ & PLR & $5 / 07 / 79$ & $8 / 20 / 79$ & 56 & N.Gas & 46 & -- & -- & Sentry 349. \\
\hline $334433 \mathrm{~N} 1021256 \mathrm{~W}$ & PLR & $4 / 05 / 79$ & $8 / 22 / 79$ & 373 & Elec. & 366 & -- & -- & Sentry 051. \\
\hline
\end{tabular}


Table 15a.--Pumpage data for irrigation sites in Lamb County, Texas

\begin{tabular}{|c|c|c|c|c|c|c|c|c|c|c|c|c|}
\hline \multirow{2}{*}{$\begin{array}{l}\text { Site } \\
\text { location }\end{array}$} & \multirow{2}{*}{$\begin{array}{l}\text { Station } \\
\text { identifier }\end{array}$} & \multicolumn{4}{|c|}{ Irrigated crop information } & \multicolumn{6}{|c|}{ We11 information } & \multirow[b]{2}{*}{ Remarks } \\
\hline & & Type & System & Acres & Source & $\begin{array}{l}\text { Depth } \\
\text { (ft) }\end{array}$ & $\begin{array}{r}\text { Water } \\
\text { level } \\
\text { (ft) }\end{array}$ & $\begin{array}{l}\text { Diam- } \\
\text { meter } \\
\text { (in). }\end{array}$ & $\begin{array}{l}\text { Pump } \\
\text { hp. }\end{array}$ & $\begin{array}{l}\text { Energy } \\
\text { source }\end{array}$ & $\begin{array}{l}\text { Energy } \\
\text { rating }\end{array}$ & \\
\hline $335239 \mathrm{~N} 1020535 \mathrm{~W}$ & 335239102053501 & Cotton & Flood & 115 & Meas. & 250 & 162 & 13 & -- & N.Gas & $\begin{array}{r}0.101 \\
.107\end{array}$ & \\
\hline $335335 \mathrm{~N} 1021543 \mathrm{~W}$ & 335335102154301 & Cotton & Flood & $\cdots$ & Meas. & 193 & 104 & 18 & -- & N.Gas & -- & Cotton crop abandoned. \\
\hline $335931 N 1021628 \mathrm{~W}$ & 335931102162801 & Cotton & Flood & 138 & Meas. & 210 & 150 & 16 & -- & N.Gas & .133 & \\
\hline $340119 N 1022443 W$ & 340119102244301 & Cotton & Flood & 7 & Meas. & 225 & 120 & 10 & -- & N.Gas & -- & Never irrigated in 1979. \\
\hline $340148 \mathrm{~N} 1022038 \mathrm{~W}$ & 340148102203801 & Cotton & -- & 77 & Meas. & 245 & 126 & 16 & -- & N.Gas & -- & Never irrigated in 1979. \\
\hline $335756 \mathrm{~N} 1022005 \mathrm{~W}$ & 335756102200501 & Cotton & Flood & 158 & Meas. & 196 & 107 & 16 & -- & N.Gas & .167 & Two wells in system. \\
\hline $340014 \mathrm{~N} 1022137 \mathrm{~W}$ & 340014102213701 & Cotton & Flood & 170 & Meas. & 206 & 117 & 16 & -- & N.Gas & -- & Three wells in system. \\
\hline $340212 \mathrm{~N} 1021557 \mathrm{~W}$ & 340212102155701 & Cotton & Flood & 40 & Meas. & 183 & 132 & 16 & -- & N.Gas & -- & \\
\hline $340212 \mathrm{N1021557 \textrm {W }}$ & 34021.2102155701 & Cotton & Flood & 48 & Meas. & 231 & 106 & 16 & -- & N.Gas & .125 & $\begin{array}{l}\text { Never irrigated cotton in } 1979 \\
\text { but irrigated other crops. }\end{array}$ \\
\hline $335348 \mathrm{~N} 1020646 \mathrm{~W}$ & 335348102064601 & Cotton & Flood & 140 & Meas. & 207 & 150 & 16 & -- & N.Gas & .137 & Two wells in system. \\
\hline $340253 \mathrm{~N} 1021137 \mathrm{~W}$ & 340253102113701 & Cotton & Flood & 75 & Meas. & 215 & 147 & 14 & - & N.Gas & .090 & \\
\hline $341150 \mathrm{~N} 1022307 \mathrm{~W}$ & 341150102230701 & $\begin{array}{l}\text { Corn } \\
\text { Cotton }\end{array}$ & Pivot & -- & Meas. & 245 & 104 & 16 & 75 & Elec. & 68 & $\begin{array}{l}\text { Corn crop was watered more than } \\
\text { the cotton. }\end{array}$ \\
\hline $335403 \mathrm{~N} 1020805 \mathrm{~W}$ & 335403102080501 & Cotton & Flood & 310 & Meas. & 200 & 142 & 16 & 10 & Elec. & 41 & Five wells in system. \\
\hline $335858 \mathrm{~N} 1021404 \mathrm{~W}$ & 335858102140401 & Cotton & Flood & 352 & Meas. & 252 & 150 & 16 & -- & N.Gas & -- & Three wells in system. \\
\hline
\end{tabular}


Table 15b.--Pumpage data for irrigation sites in Lamb County, Texas

\begin{tabular}{|c|c|c|c|c|c|}
\hline \multirow{2}{*}{$\begin{array}{c}\text { Site } \\
\text { location }\end{array}$} & \multirow{2}{*}{$\begin{array}{l}\text { Field } \\
\text { person }\end{array}$} & \multicolumn{4}{|c|}{ Discharge information } \\
\hline & & Date & $\begin{array}{l}\text { Discharge } \\
\text { (gal/min) }\end{array}$ & Method & Remarks \\
\hline $335239 \mathrm{~N} 1020535 \mathrm{~W}$ & $\begin{array}{l}\text { PLR } \\
\text { WAW }\end{array}$ & $\begin{array}{l}4 / 07 / 79 \\
8 / 06 / 79 \\
8 / 06 / 79\end{array}$ & $\begin{array}{l}260 \\
270 \\
250\end{array}$ & $\begin{array}{l}\text { Other } \\
\text { Buck. } \\
\text { Dop. }\end{array}$ & Pitot tube measurement. \\
\hline $335335 N 1021543 \mathrm{~W}$ & -- & -- & -- & -- & No discharge measurement made in 1979. \\
\hline $335931 N 1021628 \mathrm{~W}$ & $\begin{array}{l}\text { PLR } \\
\text { WAW }\end{array}$ & $4 / 04 / 79$ & $\begin{array}{l}420 \\
400\end{array}$ & $\begin{array}{l}\text { Hoff } \\
\text { Dop. }\end{array}$ & \\
\hline $340119 \times 1022443 \mathrm{~W}$ & -- & -- & -- & -- & No discharge measurement made in 1979. \\
\hline $340148 \mathrm{Ni} 022038 \mathrm{~W}$ & -- & -- & -- & -- & No discharge measurement made in 1979. \\
\hline $3357560 \mathrm{~N} 1022005 \mathrm{~W}$ & $\begin{array}{l}\text { PLR } \\
\text { WAW }\end{array}$ & $5 / 14 / 79$ & $\begin{array}{l}200 \\
210 \\
250\end{array}$ & $\begin{array}{l}\text { Buck. } \\
\text { Buck. } \\
\text { Dop. }\end{array}$ & $\begin{array}{l}\text { Well } 1 \text { of two wells. } \\
\text { Well } 2 \text { of two wells. } \\
\text { Well } 2 \text { of two wells. }\end{array}$ \\
\hline $340014 \mathrm{~N} 1022137 \mathrm{~h}$ & -- & -- & - & -- & No discharge measurement made in 1979. \\
\hline $340212 \mathrm{~N} 1021557 \mathrm{~W}$ & -- & $-\cdots$ & -- & -- & No discharge measurement made in 1979. \\
\hline $340212 \times 1021557 \mathrm{~W}$ & $\begin{array}{l}\text { PLR } \\
\text { WAW }\end{array}$ & $5 / 16 / 79$ & $\begin{array}{l}420 \\
420\end{array}$ & $\begin{array}{l}\text { Buck. } \\
\text { Dop. }\end{array}$ & \\
\hline $335343 \mathrm{~N} 1020646 \mathrm{~N}$ & WAW & $4 / 16 / 79$ & 280 & Hoff & \\
\hline $340253 \mathrm{~N} 1021137 \mathrm{~W}$ & WAW & $4 / 23 / 79$ & $\begin{array}{l}260 \\
240\end{array}$ & $\begin{array}{l}\text { Buck. } \\
\text { Dop. }\end{array}$ & \\
\hline $341150 \times 1022307 \mathrm{~W}$ & WAW & $6 / 18 / 79$ & $\begin{array}{l}750 \\
710\end{array}$ & $\begin{array}{l}\text { Other } \\
\text { Dop. }\end{array}$ & Rain gages. \\
\hline $335403 \mathrm{~N} 1020805 \mathrm{~W}$ & $\begin{array}{l}\text { PLR } \\
\text { WAW }\end{array}$ & $4 / 07 / 79$ & 310 & Buck. & \\
\hline $335858 \mathrm{~N} 1021404 \mathrm{~W}$ & -- & -- & -- & -- & No discharge measurement made in 1979. \\
\hline
\end{tabular}


Table 15c.--Pumpage data for irrigation sites in Lamb County, Texas

\begin{tabular}{|c|c|c|c|c|c|c|c|c|c|}
\hline \multirow{3}{*}{$\begin{array}{c}\text { Site } \\
\text { location }\end{array}$} & \multirow{3}{*}{$\begin{array}{r}\text { Field } \\
\text { person }\end{array}$} & \multicolumn{8}{|c|}{ Time of operation information } \\
\hline & & \multicolumn{2}{|c|}{ Date } & \multirow{2}{*}{$\begin{array}{l}\text { Sentry } \\
\text { time } \\
\text { (hours) }\end{array}$} & \multicolumn{2}{|c|}{ Energy meter } & \multicolumn{2}{|c|}{ Other } & \multirow{2}{*}{ Remarks } \\
\hline & & Begin & End & & Type & $\begin{array}{c}\text { Time } \\
\text { (hours) }\end{array}$ & Source & $\begin{array}{l}\text { Time } \\
\text { (hours) }\end{array}$ & \\
\hline $335239 \mathrm{~N} 1020535 \mathrm{~W}$ & $\begin{array}{l}\text { PLR } \\
\text { WAW }\end{array}$ & -- & $8 / 18 / 79$ & 232 & N.Gas & 215 & -- & -- & $\begin{array}{l}\text { Sentry } 037 . \\
\text { Sentry distorted. }\end{array}$ \\
\hline $335335 \mathrm{~N} 1021543 \mathrm{~W}$ & $\begin{array}{l}\text { PLR } \\
\text { WAW } \\
\text { JEC }\end{array}$ & -- & $7 / 30 / 79$ & 0 & N.Gas & 0 & -- & -- & Sentry 058. \\
\hline $335931 \mathrm{~N} 1021628 \mathrm{~W}$ & $\begin{array}{l}\text { PLR } \\
\text { WAW }\end{array}$ & $4 / 04 / 79$ & $8 / 18 / 79$ & 747 & N.Gas & 629 & -- & -- & $\begin{array}{l}\text { Sentry } 038 . \\
\text { Sentry distorted. }\end{array}$ \\
\hline 340119N1022443W & WAW & -- & $8 / 18 / 79$ & 0 & N.Gas & 0 & -- & -- & $\begin{array}{l}\text { Sentry } 044 . \\
\text { Sentry distorted. }\end{array}$ \\
\hline $340148 \mathrm{~N} 1022038 \mathrm{~W}$ & WAW & $5 / 04 / 79$ & $8 / 18 / 79$ & 0 & N.Gas & 0 & -- & -- & Sentry 313. \\
\hline $335756 \mathrm{~N} 1022005 \mathrm{~W}$ & $\begin{array}{l}\text { PLR } \\
\text { WAW }\end{array}$ & -- & $8 / 18 / 79$ & 894 & N.Gas & 1,326 & -- & -- & Sentry 074 . \\
\hline $340014 \mathrm{~N} 1022137 \mathrm{~W}$ & WAW & $4 / 04 / 79$ & $8 / 18 / 79$ & 806 & N.Gas & 770 & -- & - & $\begin{array}{l}\text { Sentry } 043 . \\
\text { Power time estimated indirectly. }\end{array}$ \\
\hline $340212 \mathrm{~N} 1021557 \mathrm{~W}$ & WAW & -- & $8 / 18 / 79$ & 0 & N.Gas & 0 & -- & -- & $\begin{array}{l}\text { Sentry } 351 . \\
\text { Timer installed late in the year. } \\
174.1 \text { mcf gas consumed in pre-water. }\end{array}$ \\
\hline $340212 \mathrm{~N} 1021557 \mathrm{~W}$ & $\begin{array}{l}\text { PLR } \\
\text { WAW }\end{array}$ & $4 / 21 / 79$ & $8 / 18 / 79$ & 291 & N.Gas & 166 & -- & -- & Sentry 376. \\
\hline $335348 \mathrm{~N} 1020646 \mathrm{~W}$ & WAW & -- & $8 / 18 / 79$ & 1,196 & N.Gas & 1,102 & - & -- & Sentry 070 . \\
\hline $340253 \mathrm{~N} 1021137 \mathrm{~W}$ & WAW & $\cdots$ & $8 / 18 / 79$ & 50 & N.Gas & 607 & -- & -- & $\begin{array}{l}\text { Sentry } 306 . \text { Field person indicates } \\
\text { that sentry time is probably } \\
\text { inaccurate. }\end{array}$ \\
\hline $341150 \times 1022307 \mathrm{~W}$ & WAW & $4 / 12 / 79$ & $8 / 18 / 79$ & 1,113 & Elec. & 1,089 & -- & -- & $\begin{array}{l}\text { Sentry } 052 . \\
\text { Pumped } 14.6 \text { ac-ft on } 88 \text { acres cotton. }\end{array}$ \\
\hline $335403 \mathrm{~N} 1020805 \mathrm{~W}$ & PLR & $4 / 04 / 79$ & $8 / 18 / 79$ & 470 & Elec. & 397 & -- & -- & $\begin{array}{l}\text { Sentry } 049 . \\
\text { Sentry distorted. }\end{array}$ \\
\hline $335858 \mathrm{~N} 1021404 \mathrm{~W}$ & WAW & $5 / 10 / 79$ & $8 / 18 / 79$ & 0 & N.Gas & 0 & -- & -- & $\begin{array}{l}\text { Sentry } 317 . \\
\text { Sentry distorted. } \\
407.3 \text { mcf gas consumed in }\end{array}$ \\
\hline
\end{tabular}


Tabie 15a.--Pumpage data for irrigation sites in Lamb County, Texas--Continued

\begin{tabular}{|c|c|c|c|c|c|c|c|c|c|c|c|c|}
\hline \multirow{2}{*}{$\begin{array}{c}\text { Site } \\
\text { location }\end{array}$} & \multirow{2}{*}{$\begin{array}{c}\text { Station } \\
\text { identifier }\end{array}$} & \multicolumn{4}{|c|}{ Irrigated crop information } & \multicolumn{6}{|c|}{ Well information } & \multirow[b]{2}{*}{ Remarks } \\
\hline & & Type & System & Acres & Source & $\begin{array}{l}\text { Depth } \\
(f t)\end{array}$ & $\begin{array}{r}\text { Water } \\
\text { level } \\
\text { (ft) }\end{array}$ & $\begin{array}{l}\text { Diam- } \\
\text { meter } \\
\text { (in) }\end{array}$ & $\begin{array}{c}\text { Pump } \\
\text { hp. }\end{array}$ & $\begin{array}{l}\text { Energy } \\
\text { source }\end{array}$ & $\begin{array}{l}\text { Energy } \\
\text { rating }\end{array}$ & \\
\hline $335555 \mathrm{~N} 1021158 \mathrm{~W}$ & 335555102115801 & Cotton & -- & 150 & Meas. & 265 & 187 & 13 & -- & N.Gas & -- & Never irrigated in 1979. \\
\hline $335732 \mathrm{~N} 1020733 \mathrm{~W}$ & 335732102073301 & Cotton & Flood & 210 & Meas. & 320 & 147 & 16 & -- & $\begin{array}{c}\text { Elec. } \\
\text { and } \\
\text { N.Gas }\end{array}$ & -- & Four wells in system. \\
\hline $340024 \mathrm{~N} 1021607 \mathrm{~W}$ & 340024102160701 & Cotton & Flood & 109 & Meas. & 190 & 130 & 16 & -- & N.Gas & -- & \\
\hline 340123N1021154W & 340123102115401 & Cotton & Flood & 51 & Meas. & 173 & 68 & 14 & -- & N.Gas & -- & \\
\hline $335336 \mathrm{~N} 1021150 \mathrm{~W}$ & 335336102115001 & Cutton & Flood & 1.45 & Meas. & 202 & 160 & 16 & -- & N.Gas & 0.193 & Two wells in system. \\
\hline $341010 \mathrm{~N} 1021845 \mathrm{~W}$ & 341010102184501 & Cotton & Flood & 231 & Meas. & 156 & 105 & 16 & $-\cdots$ & N.Gas & .294 & Two wells in system. \\
\hline $340121 \mathrm{~N} 1022030 \mathrm{~W}$ & 340121102203001 & Cotton & Sprinkler & 165 & Meas. & 234 & 103 & 18 & 20 & Elec. & 17 & \\
\hline $341144 \mathrm{~N} 1021444 \mathrm{~W}$ & 341144102144401 & Cotton & Flood & -- & Meas. & 317 & 177 & 16 & -- & N.Gas & --- & Three wells in system. \\
\hline $341747 \mathrm{~N} 1021634 \mathrm{~W}$ & 341747102163401 & Corn & Flood & -- & Meas. & 252 & 191 & 16 & -- & N.Gas & .234 & \\
\hline $335235 N 1020942 \mathrm{~W}$ & 335235102094201 & -- & Pivot & -- & Meas. & 150 & 110 & 11 & 55 & Elec. & -- & Five wells in system. \\
\hline $340032 \mathrm{~N} 1021055 \mathrm{~W}$ & 340032102105501 & Cotton & Flood & 134 & Meas. & 253 & 151 & 16 & -- & N.Gas & $\begin{array}{l}.099 \\
.124\end{array}$ & Two wells in system. \\
\hline
\end{tabular}


Table 15b.--Pumpage data for irrigation sites in Lamb County, Texxs--Continued

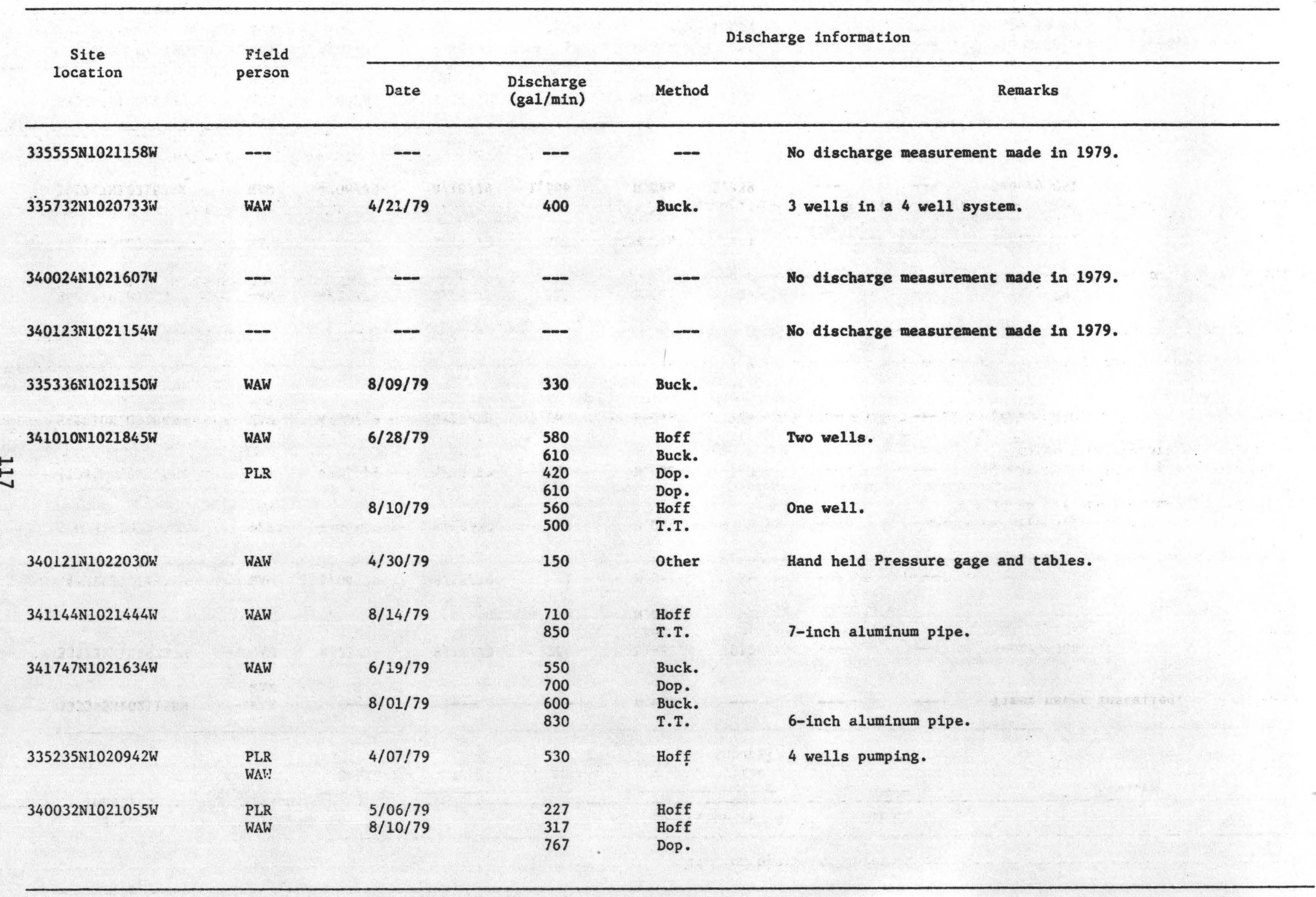


Tâble 15c.--Fumpage data for irrigation sites in Lamb County, Texas--Continued

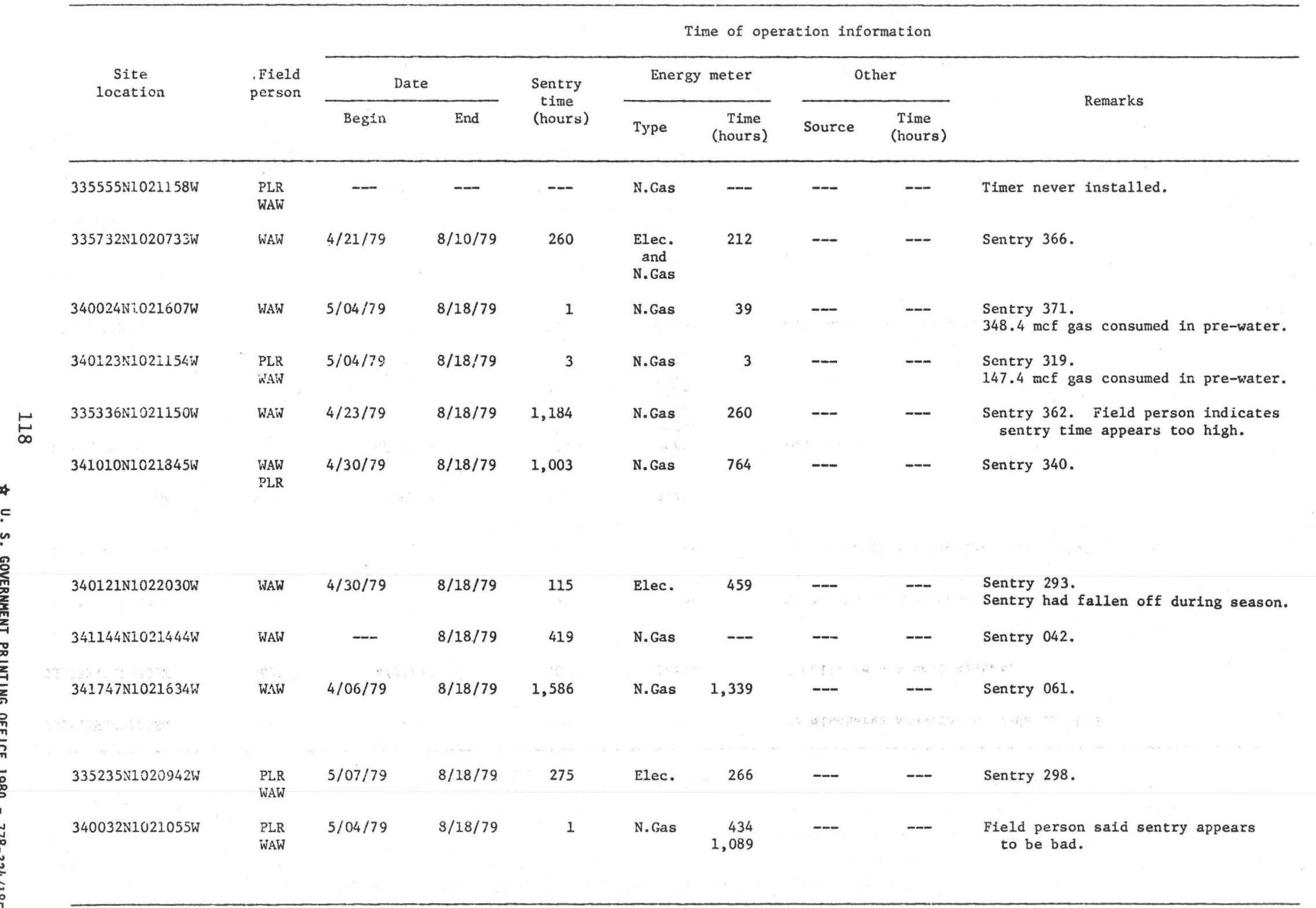

